\title{
Meflin-positive cancer-associated fibroblasts enhance tumour response to immune checkpoint blockade therapy
}

Yuki Miyai

Nagoya University

Daisuke Sugiyama

Nagoya University

Tetsunari Hase

Nagoya University

Naoya Asai

Nagoya University

Tetsuro Taki

Nagoya University

Kazuki Nishida

Nagoya University

Takayuki Fukui

Nagoya University

Toyofumi Fengshi Chen-Yoshikawa

Nagoya University

Hiroki Kobayashi

University of Adelaide

Shinji Mii

Nagoya University Graduate School of Medicine

Yukihiro Shiraki

Nagoya University

Yoshinori Hasegawa

Nagoya University

Hiroyoshi Nishikawa

National Cancer Center https://orcid.org/0000-0001-6563-9807

Yuichi Ando

Nagoya University

Masahide Takahashi

Nagoya University

Atsushi Enomoto ( $\nabla$ enomoto@iar.nagoya-u.ac.jp ) 
Nagoya University

\section{Article}

Keywords: Meflin, immunoglobulin superfamily containing leucine-rich repeat, cancer associated fibroblasts, immune checkpoint blockade, immunotherapy, non-small cell lung cancer, tumour microenvironment

Posted Date: February 25th, 2021

DOl: https://doi.org/10.21203/rs.3.rs-258152/v1

License: (9) This work is licensed under a Creative Commons Attribution 4.0 International License. Read Full License 
1 Meflin-positive cancer-associated fibroblasts enhance tumour

2 response to immune checkpoint blockade therapy

3 Yuki Miyai ${ }^{1,2}$, Daisuke Sugiyama ${ }^{3}$, Tetsunari Hase ${ }^{4}$, Naoya Asai ${ }^{1,8}$, Tetsuro Taki ${ }^{1,9}$, Kazuki

4 Nishida $^{5}$, Takayuki Fukui ${ }^{6}$, Toyofumi Fengshi Chen-Yoshikawa ${ }^{6}$, Hiroki Kobayashi ${ }^{1}$, Shinji Mii ${ }^{1}$,

5 Yukihiro Shiraki ${ }^{1}$, Yoshinori Hasegawa ${ }^{4,10}$, Hiroyoshi Nishikawa ${ }^{3,7}$, Yuichi Ando ${ }^{2}$, Masahide

6 Takahashi $^{1,11}$, and Atsushi Enomoto ${ }^{1}$

7

$8 \quad{ }^{1}$ Department of Pathology, ${ }^{3}$ Immunology, ${ }^{4}$ Respiratory Medicine, ${ }^{6}$ Thoracic Surgery, Nagoya

9 University Graduate School of Medicine, Nagoya, Japan.

${ }^{2}$ Department of Clinical Oncology and Chemotherapy, ${ }^{5}$ Center for Advanced Medicine and Clinical Research, Nagoya University Hospital, Nagoya, Japan.

${ }^{7}$ Division of Cancer Immunology, Research Institute/Exploratory Oncology Research \& Clinical Trial Center (EPOC), National Cancer Center, Tokyo/Kashiwa, Japan.

${ }^{8}$ Present address: Department of Pathology, Fujita Health University Graduate School of Medicine, Toyoake, Japan.

${ }^{9}$ Present address: Department of Pathology and Clinical Laboratories, National Cancer Center Hospital East, Kashiwa, Japan

${ }^{10}$ Present address: National Hospital Organization Nagoya Medical Center, Nagoya, Japan

${ }^{11}$ Present address: International Center for Cell and Gene Therapy, Fujita Health University, Toyoake, Japan.

Corresponding author: Atsushi Enomoto 
1 Department of Pathology, Nagoya University Graduate School of Medicine, 65 Tsurumai-Cho,

2 Showa-Ku, Nagoya 466-8550, Japan.

3 Tel: +81-52-744-2093; Fax: +81-52-744-2098; Email: enomoto@iar.nagoya-u.ac.jp

4

5 Keywords: Meflin, immunoglobulin superfamily containing leucine-rich repeat, cancer-

6 associated fibroblasts, immune checkpoint blockade, immunotherapy, non-small cell lung cancer,

7 tumour microenvironment 


\section{Abstract}

2 Cancer-associated fibroblasts (CAFs) are an integral component of the tumour microenvironment

3 (TME). Most CAFs shape the TME toward an immunosuppressive milieu and attenuate the

4 efficacy of immune checkpoint blockade (ICB) therapy. However, the detailed mechanism of

5 how heterogeneous CAFs regulate tumour response to ICB therapy has not been defined. Here,

6 we show that a novel CAF subset defined by Meflin, a glycosylphosphatidylinositol-anchored

7 protein marker of mesenchymal stromal/stem cells, is associated with survival and favourable

8 therapeutic response to ICB monotherapy in patients with non-small cell lung cancer (NSCLC).

9 The prevalence of Meflin-positive CAFs positively correlated with CD4-positive T cell

10 infiltration and vascularization within NSCLC tumours. Meflin deficiency and CAF-specific

11 Meflin overexpression resulted in defective and enhanced ICB therapy responses in xenograft

12 tumours in mice, respectively. These findings suggest the presence of a previously unknown

13 CAF subset that promotes ICB therapy efficacy, which adds to our understanding of CAF

14 functions and heterogeneity. 
2 As immune checkpoint blockade (ICB) therapy is emerging as a promising treatment strategy for

3 a wide range of cancers, understanding the mechanisms underlying tumour immunity and

4 identifying biomarkers that predict patient outcomes has been a focus of cancer research (1-9).

5 The intrinsic properties of tumour cells, mutational burdens, and their interactions with host

6 immune cells, are critical for the efficacy of ICB (6-9). However, only a subset of cancer

7 patients benefits from ICB therapy, and patients exhibit a variable response to it across cancer types (10-14). Therefore, additional studies are needed to understand the influence of tumour microenvironment (TME) and its constituents on ICB therapy response.

Cancer-associated fibroblasts (CAFs) are a major component of the TME, and proliferate in 11 the tumour stroma across multiple cancers (15-19). Recent single-cell sequencing analyses have 12 revealed that CAFs can be segregated into several clusters based on their transcriptome (20-24).

13 Two defined subpopulations of CAFs, referred to as myofibroblastic CAFs (myCAFs) and 14 inflammatory CAFs (iCAFs), were first described in pancreatic cancer $(20,21)$. Single-cell analysis of tumour stroma provided evidence of similar CAF populations in other cancer types,

16 such as breast and lung cancer (22-24). CAFs are now understood to be a major source of 17 immunosuppressive activity in the $\operatorname{TME}(25,26)$. A pioneering study showed that the CAF-S1 18 subset, which is characterised by $\alpha$-smooth muscle actin ( $\alpha$-SMA) and fibroblast activation protein (FAP) expression, is crucial for the induction of regulatory T cells to promote cancer progression and immunotherapy resistance $(22,24)$. Another study revealed that the infiltration of CAFs expressing leucine-rich repeat-containing 15 (LRRC15), whose expression was induced

22 by transforming growth factor (TGF)- $\beta$, correlated with poor response to ICB therapy across 23 multiple cancer types (27). Other studies have consistently indicated that TGF- $\beta$ signalling in 24 CAFs is correlated with immune evasion and immunotherapy failure $(28,29)$. However, a 25 complete picture of the roles of diverse CAFs in tumour immunity and responses to ICB is still 26 lacking. It is also unclear whether a specific CAF subset enhances the efficacy of ICB therapy. 
We recently described a novel CAF subset characterized by the expression of Meflin (also

known as ISLR), a glycosylphosphatidylinositol (GPI)-anchored membrane protein, in pancreatic and colorectal cancers $(30,31)$. Histological and single-cell analyses demonstrated that Meflin-positive $\left(\mathrm{Meflin}^{+}\right) \mathrm{CAFs}$ are weakly positive or negative for $\alpha$-SMA mRNA and are distinct from conventional strongly $\alpha$-SMA-positive CAFs (30). Analyses of mouse tumour models and human tissue samples suggested that the function of Meflin ${ }^{+} \mathrm{CAFs}$ is the suppression, and not progression, of cancer $(30,31)$. Biochemical analyses showed that Meflin binds to bone morphogenetic protein 7 (BMP7) to augment its signalling, which is known to inhibit the activity of TGF- $\beta$. This suggests that Meflin suppresses various TGF- $\beta$-induced responses such as tissue fibrosis (32). Based on these findings, we propose that Meflin is a specific marker of tumour-restraining CAFs ( $\mathrm{rCAFs}$ ), the existence of which has been postulated previously (33-36). However, the role of Meflin ${ }^{+} \mathrm{CAFs}$ in the tumour response to ICB therapy remains unclear.

In the present study, we showed that the proportion of Meflin ${ }^{+} \mathrm{CAFs}$ in tumour stroma correlated with a favourable response to ICB therapy in patients with non-small cell lung cancer (NSCLC) and mouse xenograft tumour models. NSCLC tumours with a high number of Meflin ${ }^{+}$ CAFs exhibited increased $\mathrm{CD} 4^{+} \mathrm{T}$ cell infiltration and areas of tumour vessels, suggesting the involvement of Meflin ${ }^{+}$CAFs in multiple aspects of the TME. To our knowledge, this is the first study revealing the presence of CAFs that enhances the response to ICB therapy. 


\section{Results}

\section{Meflin is a marker of CAFs present in the stroma of invasive NSCLC tumours}

We first examined the expression of Meflin in human lung adenocarcinoma (LUAD) tissues.

RNA in situ hybridization (ISH) analysis revealed no apparent Meflin ${ }^{+}$cells in the normal human lung tissue adjacent to the tumours (Fig. 1a, Extended Data Fig. 1). In contrast, there were several Meflin ${ }^{+}$stromal cells that infiltrated the tumour cells in invasive tumours (INV) with an extensive fibroinflammatory reaction (Fig. 1a, Extended Data Fig. 1). Interestingly, Meflin ${ }^{+}$ stromal cells were not observed in non-invasive tumours (adenocarcinoma in situ; AIS), whereas they were sparsely present in preinvasive lesions (PIL) with a lepidic growth pattern adjacent to invasive tumours (Fig. 1a, Extended Data Fig. 1). Meflin expression was also observed in stromal cells that proliferate in tumours developed in an autochthonous LUAD mouse model (KP mice), harbouring K-ras ${ }^{\mathrm{G} 12 \mathrm{D}}$ and p53 null alleles, following the administration of adenovirusexpressing Cre recombinase $(37,38)$, whereas it was hardly detected in the normal mouse lung tissue (Fig. 1b). Statistical analyses showed that the prevalence of Meflin ${ }^{+}$cells was positively correlated with an increase in the invasiveness of both human and mouse LUAD tumours (Fig. 1c, d). Further fluorescent ISH experiments showed no Meflin expression in E-cadherin ${ }^{+}$ epithelial cells, including tumour cells, $\mathrm{CD} 31^{+}$endothelial cells, or leukocyte common antigen $(\text { LCA })^{+}$leukocytes (Extended Data Fig. 2). Meflin expression was observed only in cells positive for CAF markers, such as collagen type I alpha 1 (COL1A1), $\alpha$-SMA, and podoplanin (PDPN). These data showed that Meflin is a marker of CAFs that proliferate in the invasive stages of both human and mouse LUAD.

We observed that CAFs proliferating in human NSCLC exhibited variable expression of Meflin and other CAF markers (Fig. 1e). Duplex ISH staining showed that a substantial fraction of CAFs positive for platelet-derived growth factor receptor $\alpha(\operatorname{PDGFR} \alpha)$, an established fibroblast marker (39), was also positive for Meflin. In contrast, the expression of $\alpha$-SMA, a 
marker of myCAFs $(20,21,39)$, was inversely correlated with Meflin expression; approximately $12 \%$ of CAFs expressing $\alpha$-SMA were positive for Meflin, indicating that CAFs with high Meflin expression exhibited low or negative $\alpha$-SMA expression (Fig. 1e). Meflin was expressed in approximately $33 \%$ and $12 \%$ of $\mathrm{FAP}^{+}$and $\mathrm{PDPN}^{+} \mathrm{CAFs}$, respectively. These data suggest that Meflin defines a population of CAFs, PDGFR $\alpha^{+/-} \alpha-\mathrm{SMA}^{\text {low/neg }} \mathrm{FAP}^{+/-} \mathrm{PDPN}^{\text {low/neg }}$ in human NSCLC. CAF-specific expression of Meflin was also confirmed by the analysis of single-cell RNA sequencing data of whole cells isolated from human NSCLC and distal non-malignant lung samples deposited by Lambrechts et al. (ArrayExpress accession numbers E-MTAB-6149 and EMTAB-6653, ref. 27) (Fig. 1f). These observations suggest that Meflin is a marker of a CAF subset in human NSCLC.

\section{Effect of Meflin expression in CAFs on NSCLC patients' outcome is not consistent across} different clinical stages

Given that previous studies have shown that Meflin is an rCAF marker in pancreatic and colorectal cancers $(30,31)$, we retrospectively assessed the prognostic effect of Meflin expression in CAFs on the outcomes of NSCLC patients who did not receive ICB treatment. To this end, we investigated Meflin expression by ISH in stage I-III NSCLC samples surgically resected at our institution (Nagoya University Hospital; NUH) (Extended Data Table 1, Extended Data Fig 3a). As observed in LUAD samples, Meflin expression was specifically observed in CAFs in the stroma of lung squamous cell carcinoma (LUSC) tissues (Fig. 2a). For the quantification of Meflin ${ }^{+} \mathrm{CAFs}$, we assigned all stromal cells with oval- to spindle-shaped nuclei as CAFs, excluding lymphocytes, erythrocytes, endothelial cells, and macrophages, based on their morphologies revealed by haematoxylin counterstain. Following the criteria described previously (30), we divided 88 NSCLC cases into Meflin-high ( $\geq 20 \%$ Meflin $^{+}$CAFs) and Meflin-low ( $<20 \%$ Meflin $^{+}$CAFs) groups (Fig. 2a, b). 
Consistent with the observation that the infiltration of Meflin ${ }^{+}$CAFs closely parallels the invasiveness of tumours (Fig. 1a-d), Kaplan-Meier survival analyses using the log-rank MantelCox test revealed that the Meflin-high group exhibited significantly poor overall survival (OS) after surgical resection. The pattern of disease-free survival (DFS) was similar to that of OS, albeit not statistically significant (Fig. 2c). Notably, the effect of a high number of Meflin ${ }^{+}$CAFs on poor patient outcomes was more evident in the LUAD subpopulation than in the LUSC subpopulation (Extended Data Fig. 3c). However, analyses of OS and DFS using the multivariate Cox proportional hazard regression model revealed no significant correlation between Meflin expression and the outcomes (Extended Data Table 2).

The impact of the number of Meflin ${ }^{+}$CAFs on the outcomes of patients with NSCLC was also not evident based on NSCLC cohorts obtained from the TCGA database. TCGA-LUAD and -LUSC patients were divided into Meflin-high and -low groups with an empirically determined cut-off value so that the ratio of Meflin-high and -low patients was comparable to that of the NUH cohort (Fig. 2d, Extended Data Table 3, 4, Extended Data Fig. 3b, c). These data suggested that the number of Meflin ${ }^{+} \mathrm{CAFs}$ does not affect the outcomes of patients with NSCLC, which contradicts the results of our previous studies on pancreatic and colorectal cancers $(30,31)$. Interestingly, however, although not statistically significant, high Meflin expression tended to correlate with better outcomes (OS and DFS) in advanced stage III patients, but not those in early stages (I and II) (Extended Data Fig. 3d).

\section{High infiltration of Meflin $^{+}$CAFs correlates with favourable response to ICB in NSCLC} patients

Next, we investigated the involvement of $\mathrm{Meflin}^{+} \mathrm{CAFs}$ in tumour response to ICB therapy. We conducted a retrospective observational study of 132 patients with NSCLC who had received ICB monotherapy targeting programmed cell death 1 (PD-1) (nivolumab or pembrolizumab) or 
programmed cell death 1 ligand 1 (PD-L1) (atezolizumab) at NUH (Extended Data Fig. 4a). The patients were divided into Meflin-high $\left(\geq 20 \%\right.$ Meflin $^{+}$CAFs) and Meflin-low $(<20 \%$ Meflin ${ }^{+}$CAFs) groups by ISH analysis (Extended Data Fig. 4b, c). A total of 98 patients were analysed for outcomes including objective response rate (ORR) assessed by immunotherapy Response Evaluation Criteria in Solid Tumours (iRECIST), OS, and Progression-Free Survival (PFS) (Extended Data Fig. 4a-c, Table 1). The exclusion criteria are described in Extended Data Fig. 4a.

西

Interestingly, the data showed that ORR of the Meflin-high group (40.3\%, 25 of 62 patients) was significantly higher than that of the Meflin-low group $(0 \%, 0$ of 32 patients $)(p<0.0001$, Fig. 3a). The threshold of $20 \%$ Meflin positivity in all CAFs was found to be the best criterion for predicting response to ICB monotherapy, with an area under the receiver operating characteristic curve (AUC) of 0.632 (95\% CI, 0.526-0.738) (Fig. 3b). Kaplan-Meier survival analyses using the log-rank Mantel-Cox test revealed that the Meflin-high group had a significantly favourable prognosis in both OS $(p=0.0281)$ and PFS $(p=0.0011)$ than the Meflin-low group (Fig. 3c). The positive correlation between the percentage of Meflin ${ }^{+}$CAFs and the outcomes was also shown by the multivariate Cox proportional hazard regression model (Table 2). Consistent with recent studies that showed that PD-L1 tumour proportion score (TPS) does not necessarily predict the response to ICB therapy (40-42), the analysis of PD-L1 expression in tumour cells, which was obtained with the $22 \mathrm{C} 3$ clone on the Dako Autostainer Link 48 platform, showed that PD-L1 TPS did not correlate with ORR, OS, or PFS in our NSCLC cohort (TPS < 1\%; 22.7\% ORR, TPS 1-49\%; 14.3\%, TPS $\leq 50 \% ; 38.2 \%)(\mathrm{p}=0.071$, Extended Data Fig. 5, Table 2). These data demonstrate that Meflin expression in CAFs is a predictive marker for the response to ICB in patients with NSCLC. 
1 Meflin expression in CAFs correlates with high infiltration of $\mathrm{CD4}^{+} \mathrm{T}$ cells and tumour

2 vessel area

3 We next examined the correlation between Meflin expression in CAFs and the profiles of

4 tumour-infiltrating lymphocytes (TILs). Multiplex immunofluorescence (IF) staining of the

5 specimens of 32 surgically resected NSCLC tumours who received ICB monotherapy revealed

6 that the number of $\mathrm{CD}^{+} \mathrm{T}$ cells infiltrating the stroma (interstitium), but not the tumour

7 parenchyma, was significantly higher in Meflin-high patients than in Meflin-low patients (Fig.

8 4a). In contrast, the numbers of $\mathrm{CD}^{+} \mathrm{T}$ cells and $\mathrm{CD} 4^{+} \mathrm{FoxP} 3^{+}$regulatory $\mathrm{T}$ cells were

9 comparable between the two groups (Fig. 4a). There was also no difference in the numbers of

$10 \mathrm{CD} 45 \mathrm{RO}^{+}$memory $\mathrm{CD}^{+} \mathrm{T}$ cells, $\mathrm{CD} 45 \mathrm{RO}^{+}$memory $\mathrm{CD}^{+} \mathrm{T}$ cells, or $\mathrm{CD} 20^{+} \mathrm{B}$ cells in both the

11 stroma and tumour parenchyma between the two groups (Extended Data Fig. 6).

Our previous study showed that tumours developed in the pancreas of Meflin knockout (KO) mice exhibited a decrease in tumour vessel area accompanied by changes in collagen configuration (30). Higher tumour vascularity is also associated with better tumour responses to ICB therapy in mouse models (43). Immunostaining of the NSCLC tumour samples with antiCD31 antibody showed that the Meflin-high group tumours had greater tumour vessel area than those of the Meflin-low group (Fig. 4b, Extended Data Fig. 7). These data suggest the possibility that the infiltration of $\mathrm{Meflin}^{+} \mathrm{CAFs}$ is associated with increased tumour vessel perfusion.

\section{Defective response of tumours to ICB therapy in Meflin-KO mice}

Next, we determined whether Meflin expression in CAFs is crucial for the response of tumours to ICB using C57BL/6J wild-type (WT) mice and Meflin-KO mice. We previously reported that Meflin-KO mice displayed decreased spleen weight compared to WT mice (44). Therefore, we 
1 first examined the immunophenotype of lymphocytes isolated from the spleen of Meflin-KO mice to compare it with that of WT mice. The data showed no differences in the proportions of $\mathrm{CD}^{+}, \mathrm{CD}^{+}$, and regulatory T cells (Extended Data Fig. 8a, b).

We then subcutaneously transplanted syngeneic MC-38 colorectal cancer (CRC) cells, a well-established cell line that is used to study the anti-tumour effect of ICB therapy $(2,3)$, into WT mice and Meflin-KO mice, followed by intraperitoneal administration of anti-mouse PD-1 (mPD-1) antibody or isotype control IgG on day 4, 7, and 10 after transplantation (Fig. 5a). WT mice treated with anti-mPD-1 antibody, but not isotype control IgG, had a statistically better prognosis than Meflin-KO mice (Fig. 5b). The effect of Meflin deficiency on the anti-tumour effect of mPD-1 antibody was also evaluated using a linear mixed-effect model with restricted maximum likelihood estimates, which showed that the suppressive effect of anti-mPD-1 antibody on tumour growth was significantly weakened by Meflin-KO ( $\mathrm{p}=0.0041)$, although Meflin-KO itself did not exhibit altered tumour growth $(p=0.901$, Fig. $5 c)$.

We then explored the status of TILs in tumours developed in WT and Meflin-KO mice (Extended Data Fig. 9a). Unfortunately, the infiltration of $\mathrm{CD}^{+}{ }^{+} \mathrm{T}$ cells, $\mathrm{CD} 8^{+} \mathrm{T}$ cells, and $\mathrm{CD} 25^{+} \mathrm{FoxP}^{+}$regulatory $\mathrm{T}$ cells varied across two independent experiments. Therefore, we concluded that, contrary to the analysis of human NSCLC tissues, T cell infiltration was similar between tumours developed in WT and Meflin-KO mice (Extended Data Fig. 9b). Interestingly, we found that the expression of immune checkpoint molecules in some subsets of TILs was higher in tumours of WT mice than that of Meflin-KO mice, which included T-cell immunoglobulin and mucin-domain-containing molecule 3 (TIM-3) on $\mathrm{CD}^{+}$and regulatory $\mathrm{T}$ cells, PD-1, CD25, cytotoxic T-lymphocyte associated protein 4 (CTLA-4), and inducible T-cell co-stimulator (ICOS) on $\mathrm{CD} 8^{+} \mathrm{T}$ cells (Extended Data Fig. 9c-e). Previous studies have indicated that several molecules such as PD-1 and ICOS on $\mathrm{CD}^{+} \mathrm{T}$ cells are associated with the activation of anti-tumour immunity and favourable clinical responses to ICB therapy $(9,45-47)$. 
These data suggest that Meflin expression in CAFs is associated with TIL activation in mice, but not their recruitment or infiltration into tumours.

The role of Meflin in promoting the anti-tumour effect of anti-mPD-1 antibody was also confirmed in another experimental setup, in which we orthotopically transplanted syngeneic EO771 breast cancer (BC) cells $(2,43)$ into the fourth right mammary fat pad of WT mice and Meflin-KO mice, followed by intraperitoneal administration of anti-mPD-1 antibody or control IgG on day 6, 9, and 12 after the transplantation (Fig. 5d). On day 19, a suppressive effect of anti-mPD-1 antibody on tumour volumes was observed, which was abrogated in Meflin-KO mice as indicated by the p-value of 0.0227 obtained according to the two-sided permutation Brunner-Munzel test with Holm-Bonferroni correction (Fig. 5e). The importance of Meflin expression in CAFs upon anti-mPD-1 antibody treatment was measured by the effect size (Cliff's delta) of $-0.796(95.0 \%$ CI $-1.00--0.184)$ (Fig. 5e). Consistent with the analysis of human NSCLC samples, the tumour vessel area in EO771 tumours developed in WT mice was greater than that in Meflin-KO mice (Fig. 5f). Taken together, Meflin expression in CAFs might facilitate the anti-tumour effect of anti-mPD-1 antibody by increasing the tumour vascular bed.

\section{Induced expression of Meflin in the lineage of $\mathrm{Meflin}^{+}$cells enhance the anti-tumour activity of anti-mPD-1 antibody}

The findings described above suggested that the induction of Meflin expression in CAFs can be a therapeutic strategy to enhance ICB therapy efficacy. To prove this, we generated a transgenic mouse line expressing mouse Meflin under the tetracycline response element (TRE) promoter (TRE-Meflin) (Fig. 6a). This line was then crossed with Meflin-Cre (30) and Rosa26- LSL (LoxP-stop-LoxP)-rtTA3 (third-generation reverse tetracycline-regulated transactivator) mice (JAX stock \#029617, ref. 48) to generate mice with doxycycline-induced expression of Meflin in Meflin-lineage cells. To confirm induced Meflin expression in mice carrying all three alleles 
(hereafter referred to as Meflin-TO), we administered doxycycline in drinking water $(2 \mathrm{mg} / \mathrm{mL})$ to Meflin-TO mice and transplanted MC-38 and EO771 cells subcutaneously and orthotopically, respectively, followed by the analysis of Meflin expression (Extended Data Fig. 10a). Quantitative PCR (qPCR) of the tumour tissue samples revealed that doxycycline induced Meflin expression in $\mathrm{Collal}^{+}$stromal cells, representing CAFs, in tumours developed in Meflin-TO mice administered doxycycline, but not in control mice that lack the Meflin-Cre allele (Extended Data Fig. 10b). ISH and qPCR also confirmed the induced Meflin expression in cultured CAFs isolated from tumours developed in Meflin-TO but not that of control mice (Extended Data Fig. 10c).

Consistent with the analysis of tumour vessel area of human NSCLC tissues and tumours developed in WT and Meflin-KO mice (Fig. 4b, 5f), the area of vasculature in tumours developed in doxycycline administered-Meflin-TO was significantly larger than that in MeflinTO mice not administered doxycycline and control mice that lacked the Meflin-Cre allele (Extended Data Fig. 10d).

Finally, littermates obtained by crossing Meflin-Cre, TRE-Meflin, and Rosa26-LSL-rtTA3 mice were administered doxycycline and subcutaneously transplanted with MC-38 cells, followed by intraperitoneal administration of anti-mPD-1 antibody on day 4, 7, and 10 . Genotyping of the mice was done on day 19 (Fig. 6b). The genotypes of the mice were blinded to the investigators during data acquisition and analysis. The results showed that Meflin-TO administered doxycycline and anti-mPD-1 antibody exhibited a more favourable prognosis and response than control mice (Fig. 6c, d). These data supported the notion that Meflin is a CAF marker and functionally contributes to a subset of CAFs that facilitate the anti-tumour effect of ICB therapy. 


\section{Discussion}

In the present study, we focused on the role of Meflin, a recently identified rCAF marker in pancreatic and colorectal cancers $(30,31)$, in tumour response to ICB therapy through the analyses of human NSCLC samples and xenograft tumour mouse models. Our data suggest that Meflin expression in CAFs correlates with favourable tumour response to ICB therapy, leading to the hypothesis that Meflin ${ }^{+}$CAFs promote the host anti-tumour immune response. Previous studies have shown that immunosuppressive CAFs, such as $\alpha-\mathrm{SMA}^{+} \mathrm{FAP}^{+} \mathrm{CAFs}, \mathrm{LRRC} 15^{+}$ CAFs, and TGF- $\beta$-activated CAFs, suppress anti-tumour immunity and are associated with ICB therapy failure $(24,27-29)$. We propose that a balance between the immunosuppressive CAFs and $\mathrm{Meflin}^{+} \mathrm{rCAFs}$ is crucial for determining the net response to ICB therapy (Fig. 6e).

Given our initial data that Meflin expression in CAFs correlated with the favourable outcomes of NSCLC patients treated with ICB, it was an unexpected finding that the repertoire of TILs was almost comparable between Meflin-high and -low groups, except for CD4 ${ }^{+} \mathrm{T}$ cells. Meflin expression did not affect the infiltration of $\mathrm{CD}^{+} \mathrm{T}$ cells or regulatory $\mathrm{T}$ cells, suggesting that the mechanism of action of Meflin protein and Meflin ${ }^{+}$CAFs may be different from those occurring with other molecules and modalities that directly boost tumour immunity by regulating the interactions of tumour cells with the host tissue. Interestingly, $\mathrm{CD} 4^{+} \mathrm{T}$ cell infiltration in the stroma, but not in the tumour parenchyma, correlated with the number of Meflin ${ }^{+}$CAFs in human NSCLC patients. These data may provide a mechanistic clue to the cancer-restraining role of Meflin ${ }^{+}$rCAFs. Several studies have shown the involvement of CD4 ${ }^{+}$helper T cells in tumour immunity and response to ICB (49-52), which is distinct from their prominent role in inducing tumour-reactive cytotoxic T cells (53-56). Notably, the repertoire of TILs was comparable between tumours developed in WT and Meflin-KO mice in a xenograft tumour model, suggesting that $\mathrm{CD}^{+} \mathrm{T}$ cell infiltration depends on the overall function of Meflin ${ }^{+} \mathrm{CAFs}$, but not specifically the function of Meflin. 
We previously identified BMP7 as a ligand of Meflin and reported that it augments BMP7 signalling, which suppresses TGF- $\beta$ signalling and tissue fibrosis (32). Consistent with this, the induction of cardiac fibrosis and pancreatic carcinogenesis in Meflin-KO mice resulted in enhanced fibrosis and infiltration of $\alpha-\mathrm{SMA}^{+}$myofibroblasts or CAFs compared to WT mice (30, 32). Interestingly, Meflin-KO hearts exhibit an increased stiffness compared to WT hearts in a cardiac fibrosis model, leading to the hypothesis that the fundamental function of Meflin is to inhibit tissue fibrosis and interstitial pressure elevation in chronic disease conditions (32). Consistent with this, the present data showed that the tumour vessel areas correlated with Meflin expression in CAFs in both human NSCLC tissues and mouse models. Although not proven in the present study, an intriguing hypothesis is that Meflin-mediated suppression of tissue fibrosis or decrease in interstitial pressure facilitates tumour vessel perfusion and therapeutic antibody delivery. In addition, the involvement of Meflin in controlling the enhanced permeability and retention (EPR) effect, which refers to the ability of macromolecules such as anti-PD-1 antibodies to accumulate in the tumour tissue $(57,58)$, will be a subject of future research.

An appealing feature of Meflin is that none (0\%) of the NSCLC patients in our institution with low Meflin expression in CAFs responded to ICB therapy. These data suggest that the number of Meflin ${ }^{+}$CAFs could be a marker for identifying patients who will not benefit from ICB therapy. The present study also showed that the induced expression of Meflin in CAFs increased the anti-tumour effect of anti-mPD-1 antibody in a transgenic mouse model. The data implied that the augmentation of Meflin expression in CAFs could be a therapeutic strategy to increase the efficacy of ICB therapy. In relation to this issue, we previously showed that calcipotriol, a vitamin D analogue, induced the upregulation of Meflin expression in CAFs isolated from human pancreatic cancer (30). Our findings are consistent with a previous study that showed that calcipotriol administration induced changes in the TME with an increase in the delivery of chemotherapeutic agents to the tumour in a pancreatic cancer mouse model (59). Ongoing clinical trials have investigated the use of vitamin D analogues in combination with 
1 immune checkpoint inhibitors and chemotherapeutic agents (clinicaltriaol.gov) (60). It would be 2 interesting to study how Meflin is involved in vitamin D-mediated remodelling of the TME and 3 the increased efficiency of ICB therapy in clinical settings.

$4 \quad$ In conclusion, we identified a CAF subset marked by Meflin expression and found that its 5 prevalence is associated with a favourable response to ICB therapy in NSCLC patients and 6 xenograft tumour mouse models. Induction of Meflin expression in CAFs augmented the tumour 7 response to ICB therapy in mice. Together with other studies that identified CAF subsets that 8 suppress anti-tumour immunity and are associated with ICB treatment failure, we propose that 9 the heterogeneity of CAFs determines the net response of tumours to ICB therapy. 


\section{Acknowledgements}

2 The results shown here are in part based on data generated by the TCGA Research Network:

3 https://www.cancer.gov/tcga. We thank Kaori Ushida and Kozo Uchiyama (Nagoya University)

4 for their support in immunostaining and tissue preparation and the staff of the Division of

5 Experimental Animals (Nagoya University) for their support in animal experiments. This work

6 was supported by a Grant-in-Aid for Scientific Research (B) (18H02638 to A.E., 20H03467 to

7 M.T., $20 H 03528$ to Y.A.) and a Grant-in-Aid for Research Activity Start-up (20K22807 to Y.M.)

8 commissioned by the Ministry of Education, Culture, Sports, Science and Technology of Japan;

9 Nagoya University Hospital Funding for Clinical Research (to A.E.); AMED-CREST (Japan

10 Agency for Medical Research and Development, Core Research for Evolutional Science and

11 Technology; 20gm0810007h0105 and 20gm1210009s0102 to A.E.), the Project for Cancer

12 Research and Therapeutic Evolution (P-CREATE) from AMED (20cm0106377h0001 to A.E.),

13 and Aichi Cancer Research Foundation (to Y.M.). We thank Editage [http://www.editage.com]

14 for editing and reviewing this manuscript for English language.

\section{Author contributions}

17 Y.M. designed and performed the experiments, analysed the data, and wrote the manuscript. D.S.

18 designed and performed the experiments, and analysed the data. T.H., T.F., T.F.C-Y., and Y.H.

19 provided the clinical samples and intellectual input. N.A. and H.K. generated the TRE-Meflin

20 mouse line. T.T. S.M. and Y.S. generated the KP mice and assisted with histological analysis.

21 K.N. designed the statistical analyses and wrote the manuscript. H.N., Y.A., and M.T. directed

22 the project and provided intellectual input. A.E. directed the project and wrote the manuscript. 


\section{Competing Interests statement}

2 Y.M. is the primary inventor on a pending patent PCT/JP2019/004521 belonging to Nagoya

3 University. T.H. has received honoraria from AstraZeneca, Ono Pharmaceutical, Chugai

4 Pharmaceutical, and Bristol-Myers Squibb and received research funding from Boehringer

5 Ingelheim, and Taiho Pharmaceutical outside the scope of this work. Y.H. has received honoraria

6 from AstraZeneca, Ono Pharmaceutical, Chugai Pharmaceutical and Taiho Pharmaceutical, and

7 received research funding from Ono Pharmaceutical, Chugai Pharmaceutical, and Taiho

8 Pharmaceutical outside the scope of this work. H.N. received honoraria and research funding

9 from Ono Pharmaceutical, Chugai Pharmaceutical, MSD and Bristol-Myers Squibb, and research

10 funding from Taiho Pharmaceutical, Daiichi-Sankyo, Kyowa Kirin, Zenyaku Kogyo, Oncolys

11 BioPharma, Debiopharma, Asahi-Kasei, Sysmex, Fujifilm, SRL, Astellas Pharmaceutical,

12 Sumitomo Dainippon Pharma and BD Japan outside of this study. Y.A. has received honoraria

13 from Chugai Pharmaceutical and received research funding from Novartis, Ono Pharmaceutical,

14 Chugai Pharmaceutical, Bayer Yakuhin, and Yakult Honsha outside the scope of this work. The

15 other authors declare no competing interests. 


\section{References}

1. Smith, S. M. et al. Clinical Cancer Advances 2021: ASCO's Report on Progress Against Cancer. J. Clin. Oncol. JCO.20.03420 (2021).

2. House, I. G. et al. Macrophage-derived CXCL9 and CXCL10 are required for antitumor immune responses following immune checkpoint blockade. Clin. Cancer Res. 26, 487-504 (2020).

3. Mager, L. F. et al. Microbiome-derived inosine modulates response to checkpoint inhibitor immunotherapy. Science 369, 1481-1489 (2020).

4. Hegde, S. et al. Dendritic cell paucity leads to dysfunctional immune surveillance in pancreatic cancer. Cancer Cell 37, 289-307.e9 (2020).

11 5. Oh, S. A. et al. PD-L1 expression by dendritic cells is a key regulator of T-cell immunity in 12 cancer. Nat. Cancer 1, 681-691 (2020).

6. Kumagai, S. et al. An oncogenic alteration creates a microenvironment that promotes tumor progression by conferring a metabolic advantage to regulatory T cells. Immunity 53, 187-203.e8 15 (2020).

16 7. Rizvi, N. A. et al. Mutational landscape determines sensitivity to PD-1 blockade in non-small 17 cell lung cancer. Science 348, 124-128 (2015).

8. Yarchoan, M., Hopkins, A. \& Jaffee, E. M. Tumor mutational burden and response rate to PD1 inhibition. N. Engl. J. Med. 377, 2500-2501 (2017).

9. Kumagai, S. et al. The PD-1 expression balance between effector and regulatory T cells predicts the clinical efficacy of PD-1 blockade therapies. Nat. Immunol. 21, 1346-1358 (2020). 10. Carbognin, L. et al. Differential activity of nivolumab, pembrolizumab, and MPDL3280A according to the tumor expression of programmed death-ligand-1 (PD-L1): sensitivity analysis of trials in melanoma, lung, and genitourinary cancers. PLoS One 10, e0130142 (2015).

11. Amraee, A. et al. Efficacy of nivolumab as a checkpoint inhibitor drug on survival rate of patients with relapsed/refractory classical Hodgkin lymphoma: a meta-analysis of prospective clinical study. Clin. Transl. Oncol. 21, 1093-1103 (2019).

12. Iacovelli, R. et al. Immunotherapy versus standard of care in metastatic renal cell carcinoma A systematic review and meta-analysis. Cancer Treat. Rev. 70, 112-117 (2018).

13. Lin, Z. et al. PD-1 antibody monotherapy for malignant melanoma: A systematic review and meta-analysis. PLoS One 11, e0160485 (2016).

14. Voutsadakis, I. A. PD-1 inhibitors monotherapy in hepatocellular carcinoma: Meta-analysis and systematic review. Hepatobiliary Pancreat. Dis. Int. 18, 505-510 (2019).

34 15. Sahai, E. et al. A framework for advancing our understanding of cancer-associated 35 fibroblasts. Nat. Rev. Cancer 20, 174-186 (2020). 
16. Kalluri, R. The biology and function of fibroblasts in cancer. Nat. Rev. Cancer 16, 582-598 (2016).

17. Kobayashi, H. et al. Cancer-associated fibroblasts in gastrointestinal cancer. Nat. Rev. Gastroenterol. Hepatol. 16, 282-295 (2019).

18. Miyai, Y., Esaki, N., Takahashi, M. \& Enomoto, A. Cancer-associated fibroblasts that restrain cancer progression: Hypotheses and perspectives. Cancer Sci. 111, 1047-1057 (2020).

19. Piersma, B., Hayward, M. K. \& Weaver, V. M., Fibrosis and cancer: A strained relationship. Biochim. Biophys. Acta Rev. Cancer 1873, 188356 (2020)

20. Öhlund, D. et al. Distinct populations of inflammatory fibroblasts and myofibroblasts in pancreatic cancer. J. Exp. Med. 214, 579-596 (2017).

21. Elyada, E. et al. Cross-species single-cell analysis of pancreatic ductal adenocarcinoma reveals antigen-presenting cancer-associated fibroblasts. Cancer Discov. 9, 1102-1123 (2019)

22. Costa, A. et al. Fibroblast heterogeneity and immunosuppressive environment in human breast cancer. Cancer Cell 33, 463-479.e10 (2018).

23. Lambrechts, D. et al. Phenotype molding of stromal cells in lung tumor microenvironment Nat. Med. 24, 1277-1289 (2018).

24. Kieffer, Y. et al. Single-cell analysis reveals fibroblast clusters linked to immunotherapy resistance in cancer. Cancer Discov. 10, 1330-1351 (2020)

25. Barrett, R. L. \& Puré, E. Cancer-associated fibroblasts and their influence on tumor immunity and immunotherapy. eLife 9, e57243 (2020).

26. Baker, A. T., Abuwarwar, M. H., Poly, L., Wilkins, S. \& Fletcher, A. L. Cancer-associated fibroblasts and T cells: From mechanisms to outcomes. J. Immunol. 206, 310 (2021).

27. Dominguez, C. X., et al. Single-cell RNA sequencing reveals stromal evolution into LRRC15 + myofibroblasts as a determinant of patient response to cancer immunotherapy. Cancer Discov. 10, 232-253 (2020).

28. Mariathasan et al. TGF $\beta$ attenuates tumour response to PD-L1 blockade by contributing to exclusion of T cells. Nature 554, 544-548 (2018).

29. Chakravarthy, A., Khan, L., Bensler, N. P., Bose, P. \& De Carvalho, D. D. TGF- $\beta$-associated extracellular matrix genes link cancer-associated fibroblasts to immune evasion and immunotherapy failure. Nat. Commun. 9, 4692 (2018).

30. Mizutani, Y. et al. Meflin-positive cancer-associated fibroblasts inhibit pancreatic carcinogenesis. Cancer Res. 79, 5367-5381 (2019).

31. Kobayashi, H. et al. (In Press) The balance of stromal BMP signaling mediated by GREM1 and ISLR drives colorectal carcinogenesis. Gastroenterology (2020)

32. Hara, A. et al. Roles of the mesenchymal stromal/stem cell marker Meflin in cardiac tissue repair and development of diastolic dysfunction Circ. Res. 125, 414-430 (2019). 
33. Rhim, A. D. et al. Stromal elements act to restrain, rather than support, pancreatic ductal adenocarcinoma. Cancer Cell 25, 735-747 (2014).

34. Özdemir, B. C. et al. Depletion of carcinoma-associated fibroblasts and fibrosis induces immunosuppression and accelerates pancreas cancer with reduced survival. Cancer Cell 25, 719-734 (2014).

35. Lee, J. J. et al. Stromal response to Hedgehog signaling restrains pancreatic cancer progression Proc. Natl Acad. Sci. USA 111, E3091-E3100 (2014).

36. Shin, K. et al. Hedgehog signaling restrains bladder cancer progression by eliciting stromal production of urothelial differentiation factors. Cancer Cell 26, 521-533 (2014).

37. Taki, T. et al. CD109 regulates in vivo tumor invasion in lung adenocarcinoma through TGF$\beta$ signaling. Cancer Sci. 111, 4616-4628 (2020).

38. DuPage, M., Dooley, A. L. \& Jacks, T. Conditional mouse lung cancer models using adenoviral or lentiviral delivery of Cre recombinase. Nat. Protoc. 4, 1064-1072 (2009).

39. Han, C., Liu, T. \& Yin, R. Biomarkers for cancer-associated fibroblasts. Biomark. Res 8, 64 (2020).

40. Carbone, D. P. et al. First-line nivolumab in stage IV or recurrent non-small-cell lung cancer. N. Engl. J. Med. 376, 2415-2426 (2017).

41. Shen, X. \& Zhao, B. Efficacy of PD-1 or PD-L1 inhibitors and PD-L1 expression status in cancer: meta-analysis. BMJ 362, k3529 (2018).

42. Lu, S. et al. Comparison of biomarker modalities for predicting response to PD-1/PD-L1 checkpoint blockade: A systematic review and meta-analysis JAMA Oncol. 5, 1195-1204 (2019).

43. Zheng, X. et al. Increased vessel perfusion predicts the efficacy of immune checkpoint blockade. J. Clin. Invest. 128, 2104-2115 (2018).

44. Maeda, K. et al. Identification of Meflin as a potential marker for mesenchymal stromal cells Sci. Rep. 6, 22288 (2016).

45. Gros, A. et al. PD-1 identifies the patient-specific CD8+ tumor-reactive repertoire infiltrating human tumors. J. Clin. Invest. 124, 2246-2259 (2014).

46. Gros, A. et al. Prospective identification of neoantigen-specific lymphocytes in the peripheral blood of melanoma patients Nat. Med. 22, 433-438 (2016).

47. Xiao, Z., Mayer, A. T., Nobashi, T. W. \& Gambhir, S. S. ICOS is an indicator of T-cellmediated response to cancer immunotherapy. Cancer Res. 80, 3023-3032 (2020).

48. Dow, L. E. et al. Conditional reverse tet-transactivator mouse strains for the efficient induction of TRE-regulated transgenes in mice. PLoS One 9, e95236 (2014).

49. Liu, M. et al. TGF- $\beta$ suppresses type 2 immunity to cancer. Nature 587, 115-120 (2020).

50. Li, S. et al. Cancer immunotherapy via targeted TGF- $\beta$ signalling blockade in TH cells. Nature 587, 121-125 (2020). 
1 51. Zuazo, M. et al. Functional systemic CD4 immunity is required for clinical responses to PD-

2 L1/PD-1 blockade therapy. EMBO Mol. Med. 11, e10293 (2019).

3 52. Kagamu, H. et al. CD4 T-cell immunity in the peripheral blood correlates with response to

4 anti-PD-1 therapy. Cancer Immunol. Res. 8, 334-344 (2020).

5 53. Kreiter, S. et al. Mutant MHC class II epitopes drive therapeutic immune responses to cancer.

6 Nature 520, 692-696 (2015).

7 54. Sahin, U. et al. Personalized RNA mutanome vaccines mobilize poly-specific therapeutic

8 immunity against cancer. Nature 547, 222-226 (2017).

9 55. Borst, J., Ahrends, T., Bąbała, N., Melief, C. J. M. \& Kastenmüller, W. CD4+ T cell help in 10 cancer immunology and immunotherapy. Nat. Rev. Immunol. 18, 635-647 (2018).

11 56. Zuazo, M. et al. Systemic CD4 immunity as a key contributor to PD-L1/PD-1 blockade

12 immunotherapy efficacy. Front. Immunol. 11, 586907 (2020).

13 57. Matsumura, Y. \& Maeda, H. A new concept for macromolecular therapeutics in cancer 14 chemotherapy: Mechanism of tumoritropic accumulation of proteins and the antitumor agent

15 Smancs. Cancer Res. 46, 6387-6392 (1986).

16 58. Matsumura, Y. Cancer stromal targeting therapy to overcome the pitfall of EPR effect. Adv.

17 Drug Deliv. Rev. 154-155, 142-150 (2020).

18 59. Sherman, M. H. et al. Vitamin D receptor-mediated stromal reprogramming suppresses

19 pancreatitis and enhances pancreatic cancer therapy. Cell 159, 80-93 (2014).

20 60. Gong, J. et al. Combination systemic therapies with immune checkpoint inhibitors in 21 pancreatic cancer: overcoming resistance to single-agent checkpoint blockade. Clin. Transl. Med. 227, e32 (2018). 
(a) Tissue sections derived from non-invasive (AIS) and invasive (INV) LUAD tumours were

stained for H\&E (top panels) and Meflin mRNA by ISH (lower panels). Preinvasive lesions adjacent to the invasive tumour (PIL) and adjacent normal tissue (AN) were also examined.

Boxed areas were magnified in lower panels. Black and open arrowheads denote $\mathrm{Meflin}^{+}$stromal cells and Meflin' macrophages that phagocytise foreign material, respectively. Areas filled with yellow indicate tumour parenchyma comprised of tumour cells $(\mathrm{T})$.

(b) Preinvasive (PIL) and invasive (INV) lesions of a tumour developed in the autochthonous LUAD mouse model (KP mice) were examined for Meflin expression by ISH. Meflin ${ }^{+}$stromal $^{2}$ cells (arrowheads) were found in the INV and PIL lesion, but not in adjacent normal tissue (AN).

(c, d) Quantification of the percentage of Meflin $^{+}$cells in all cells with oval- to spindle-shaped nuclei found in the stroma of the normal (AN), non-invasive (AIS), preinvasive (PIL) and invasive (INV) cases and lesions of human LUAD cases (c) and the KP mouse model (d), respectively. All stromal cells found in high-power fields (400x) of the indicated number of cases were evaluated.

(e) Duplex ISH for Meflin (red) and other CAF markers (green) showed that Meflin is variably co-expressed with other established CAF markers (PDGFRA, ACTA2, FAP, and PDPN) in CAFs of human NSCLC. Green, yellow, and red arrow heads denote cells that are single-positive for the indicated CAF markers, double-positive for the indicated CAF markers and Meflin, and single-positive for Meflin, respectively. All stromal cells that were positive for ISH signals in high-power fields from 3 independent NSCLC tumours were evaluated and quantified.

(f) A single-cell analysis showing the specific expression of Meflin in fibroblasts in human NSCLC and distal non-malignant lung samples. The magnified image of the fibroblast fraction 
1 showed that Meflin is expressed in some but not all fibroblasts, and that $\mathrm{Meflin}^{+} \mathrm{CAFs}$ constitute 2 a unique CAF subset distinct from $\alpha-\mathrm{SMA}^{+} \mathrm{CAFs}$. 


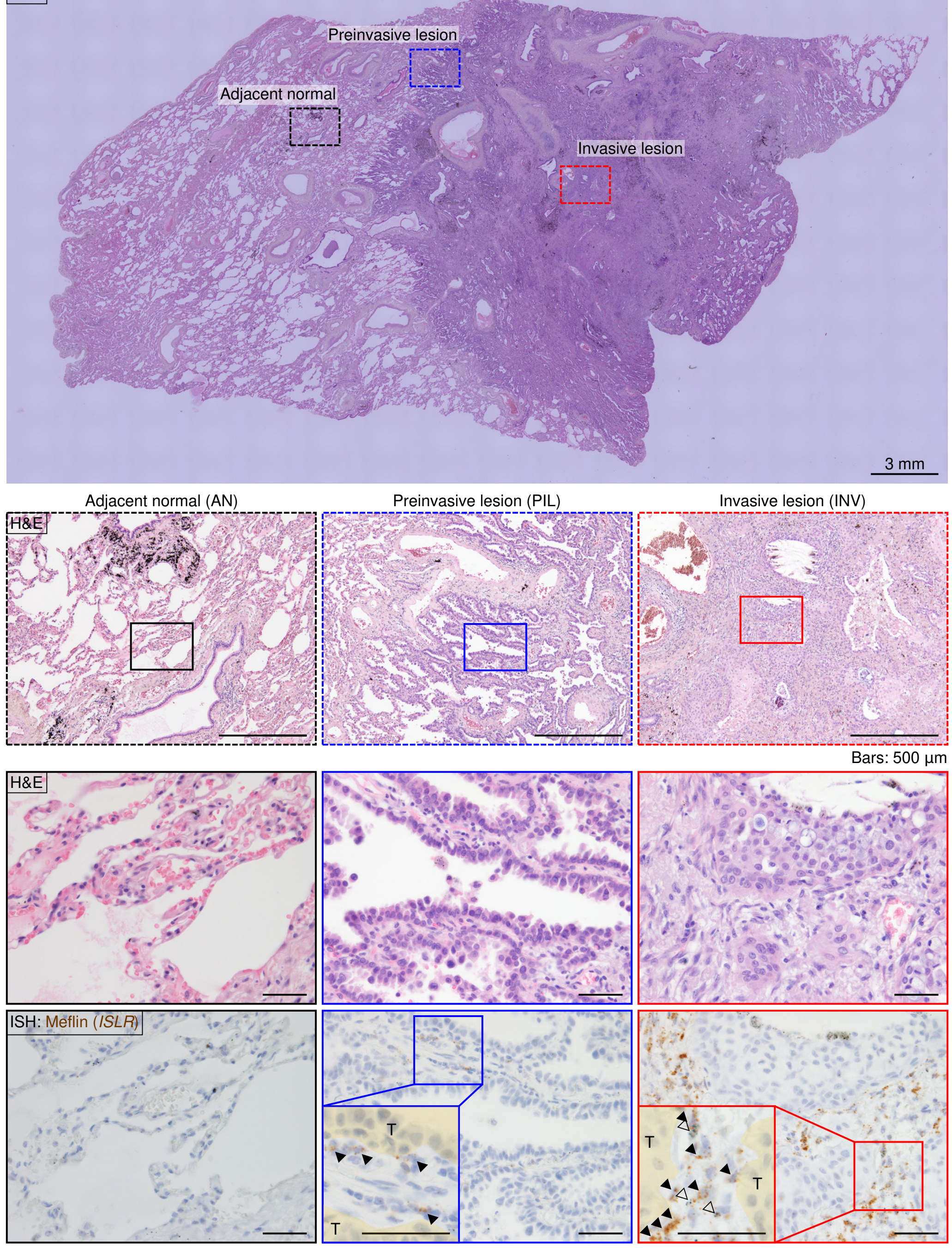

Bars: $50 \mu \mathrm{m}$

\section{ExFig. 1 Miyai et al.}


1 Extended Data Fig. 1. Meflin expression in a case of human LUAD

2 A tissue section from a human LUAD case was stained for H\&E staining (upper panels) and

3 Meflin mRNA by ISH (lowest panel). Dashed boxed areas (preinvasive and invasive lesions and

4 adjacent normal tissue) in the top panel are magnified in the lower panels. Solid-line boxed areas

5 in the middle panels are further magnified in the lower panels, which show serial sections stained

6 for H\&E and Meflin mRNA by ISH. Meflin ${ }^{+}$stromal cells were not found in the adjacent normal

7 tissue (far left lower panel), whereas a small number of Meflin ${ }^{+}$cells (arrow heads) was observed

8 in the stroma of the preinvasive lesion (lower middle panel, blue box). In the invasive lesion,

9 there were many Meflin ${ }^{+}$cells in the stroma, supporting the notion that the infiltration of Meflin

10 cells is associated with tumour invasion (far right lower panel). Open arrowheads denote

11 macrophages that phagocytize foreign materials.

12 
a

Non-CAF markers / Meflin (ISLR)

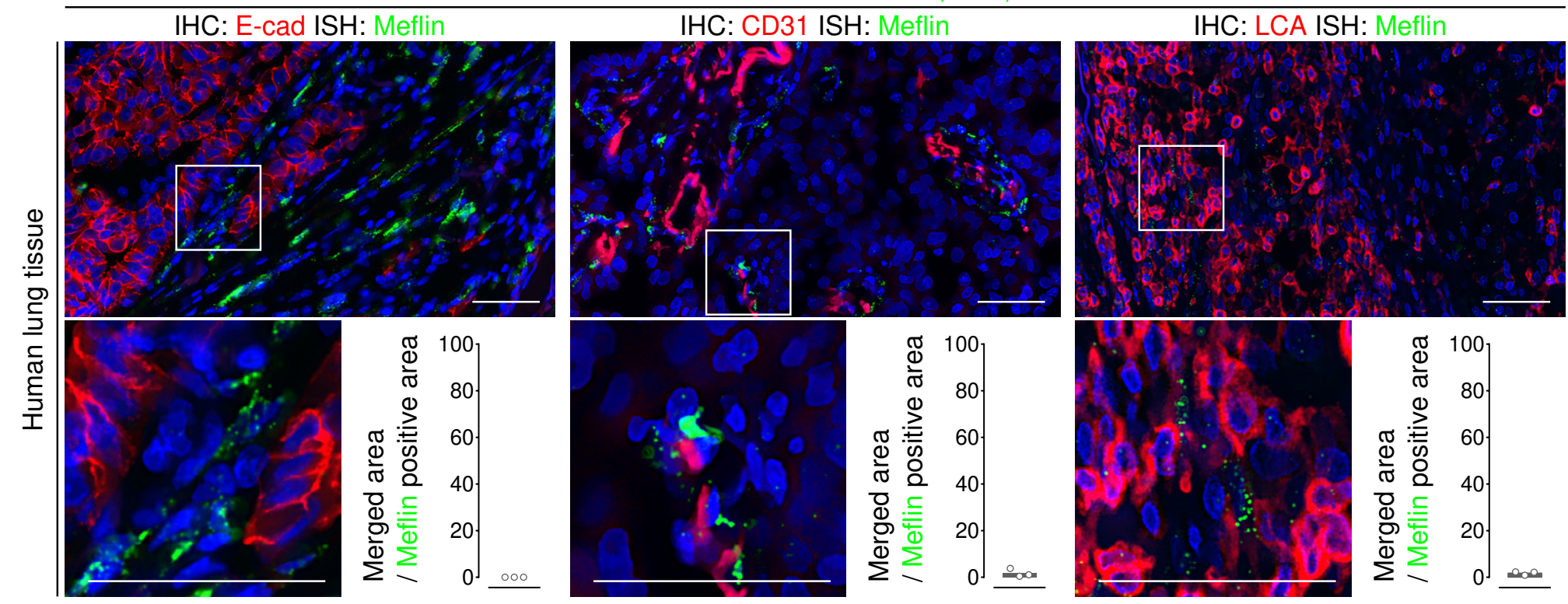

b

CAF markers / Meflin (ISLR)

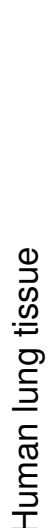

IHC: Col1a1 ISH: Meflin

IHC: aSMA ISH: Meflin
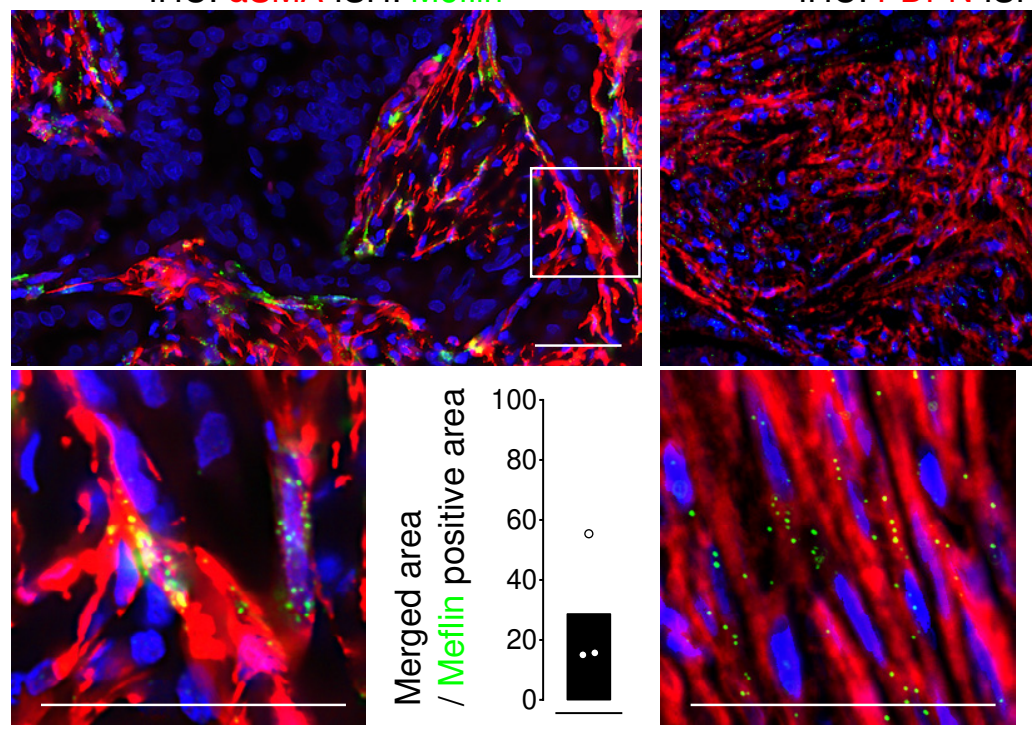

IHC: PDPN ISH: Meflin

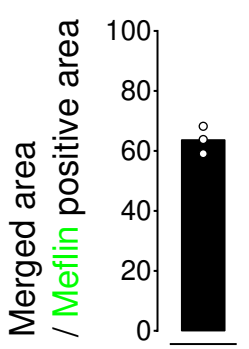

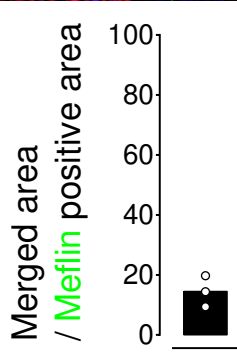

Bars: $50 \mu \mathrm{m}$

ExFig.2 Miyai et al. 
1 Extended Data Fig. 2. Meflin is expressed in stromal fibroblasts but not in other types of

2 cell in human NSCLC

3 (a) Tissue sections prepared from a human NSCLC sample were stained for the epithelial cell

4 marker E-cadherin, the endothelial cell marker CD31, and the leukocyte marker LCA by

5 immunofluorescence (IF) (red) and Meflin mRNA by ISH using RNAscope technology (green).

6 Note that no Meflin ${ }^{+}$cells were positive for E-cadherin, CD31, and LCA. The graphs in the

7 insets show the ratios of the merged areas to those of the Meflin ${ }^{+}$areas.

8 (b) Tissue sections prepared from a human NSCLC sample were stained for the CAF markers

9 Colla1, $\alpha$-SMA, and podoplanin by IF (red) with Meflin mRNA detection by ISH using

10 RNAscope technology (green). The graphs in the insets indicate the ratios of the merged areas to

11 those of the Meflin ${ }^{+}$areas.

12

13 
Fig. 2. Infiltration of Meflin ${ }^{+}$CAFs is associated with poor outcomes of NSCLC patients

(a) Representative images of Meflin-high and -low cases of invasive adenocarcinoma (left) and squamous cell carcinoma (right). Dashed boxed areas were magnified in middle panels, which showed serial sections stained for H\&E and Meflin mRNA by ISH. In the images of Meflin ISH, boxed areas were magnified in the lowest panels. Arrowheads indicate Meflin ${ }^{+}$CAFs.

(b) Distribution of NSCLC patients stratified by the percentage of Meflin ${ }^{+}$CAFs in all stromal cells. The number of Meflin ${ }^{+}$CAFs was counted in randomly selected five high-power microscopic fields. The proportion of Meflin ${ }^{+}$CAFs was represented as the ratio of Meflin ${ }^{+}$ CAFs to all stromal cells with oval to spindle-shaped nuclei. Meflin-high was defined as $\geq 20 \%$ of stromal cells positive for Meflin.

(c) Comparison of the outcomes of Meflin-high and -low NSCLC patients in our institution. Eighty-eight cases were classified according to Meflin expression, and OS (left) and DFS (right) of the cases were plotted by Kaplan-Meier analysis.

(d) Effects of Meflin expression on NSCLC patients' outcomes analysed by using data sets available in the TCGA database. Seven hundred fifty-eight cases were classified into Meflin-high and -low groups, and OS (left) and PFS (right) were plotted by Kaplan-Meier analysis. Cut-off values were set at the top 33 and 80 percentiles for the expression of Meflin in LUAD and LUSC, respectively. 
a

Patients with pathologically confirmed NSCLC those received operation at NUH in 2010: 147 pts

Adenocarcinoma in situ: 20 pts

Neoadjuvant chemotherapy: 11 pts

Multiple cancer: 26 pts

Inappropriate / shortage of specimens: 2 pts

Survival evaluable patients: 88 pts b

NSCLC Patients in TCGA-
LUAD cohort: 586 pts

NSCLC Patients in TCGA-

LUSC cohort: 511 pts

C

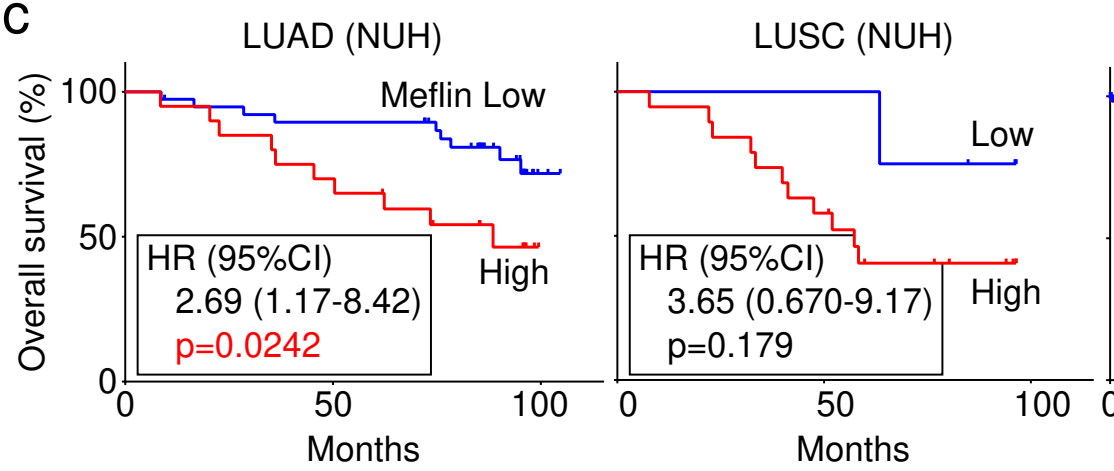

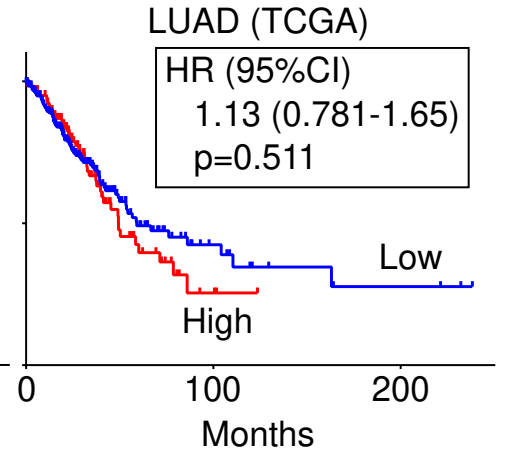

Months

\begin{tabular}{|rcr|}
\hline $69 \mathrm{pts}$ & No RNA-seq data & $10 \mathrm{pts}$ \\
$44 \mathrm{pts}$ & Positive surgical margins & $39 \mathrm{pts}$ \\
$93 \mathrm{pts}$ & Prior malignancy & $70 \mathrm{pts}$ \\
$26 \mathrm{pts}$ & Stage IV & $7 \mathrm{pts}$ \\
$3 \mathrm{pts}$ & Neoadjuvant therapy & $7 \mathrm{pts}$ \\
$2 \mathrm{pts}$ & Recurrent sample & $0 \mathrm{pts}$ \\
$71 \mathrm{pts}$ & No survival data & $13 \mathrm{pts}$ \\
\hline
\end{tabular}

371 pts Overall survival evaluable patients

387 pts

d

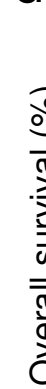

Stage I (TCGA)

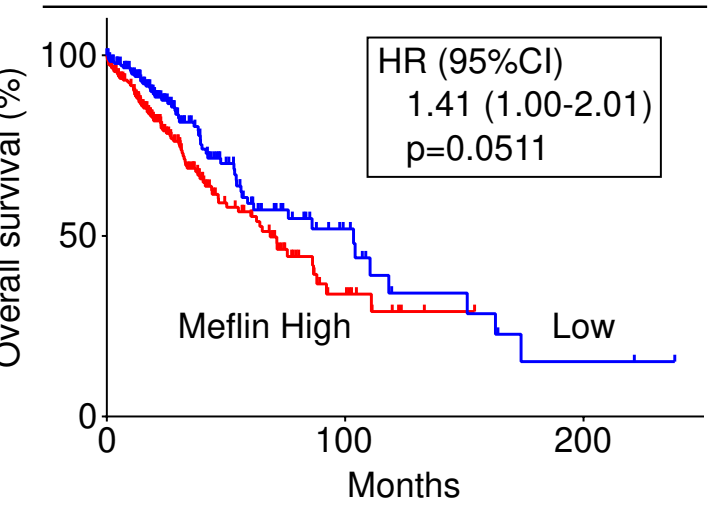

$\frac{2}{0}$

$\sum_{0}^{\infty}$

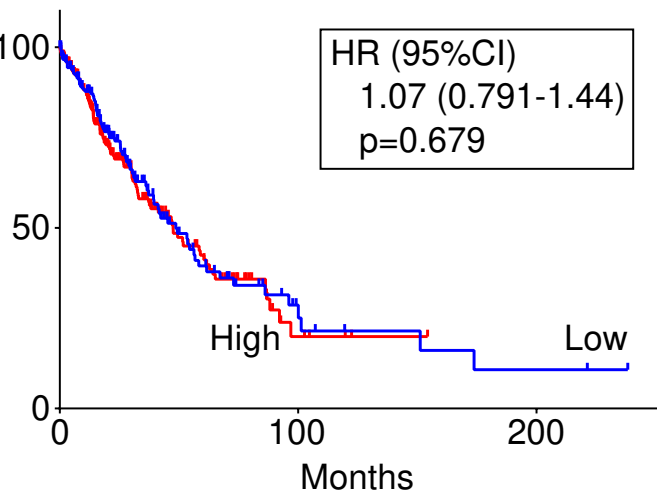

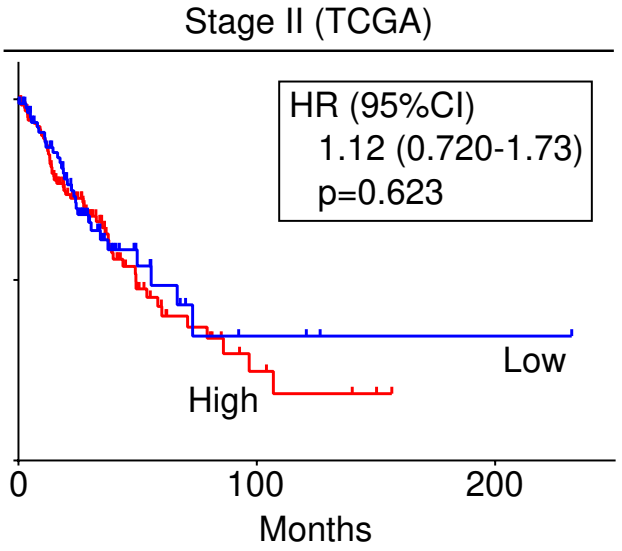

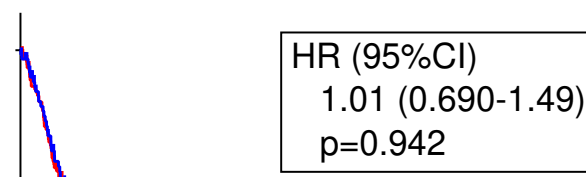

ExFig.3 Miyai et al.
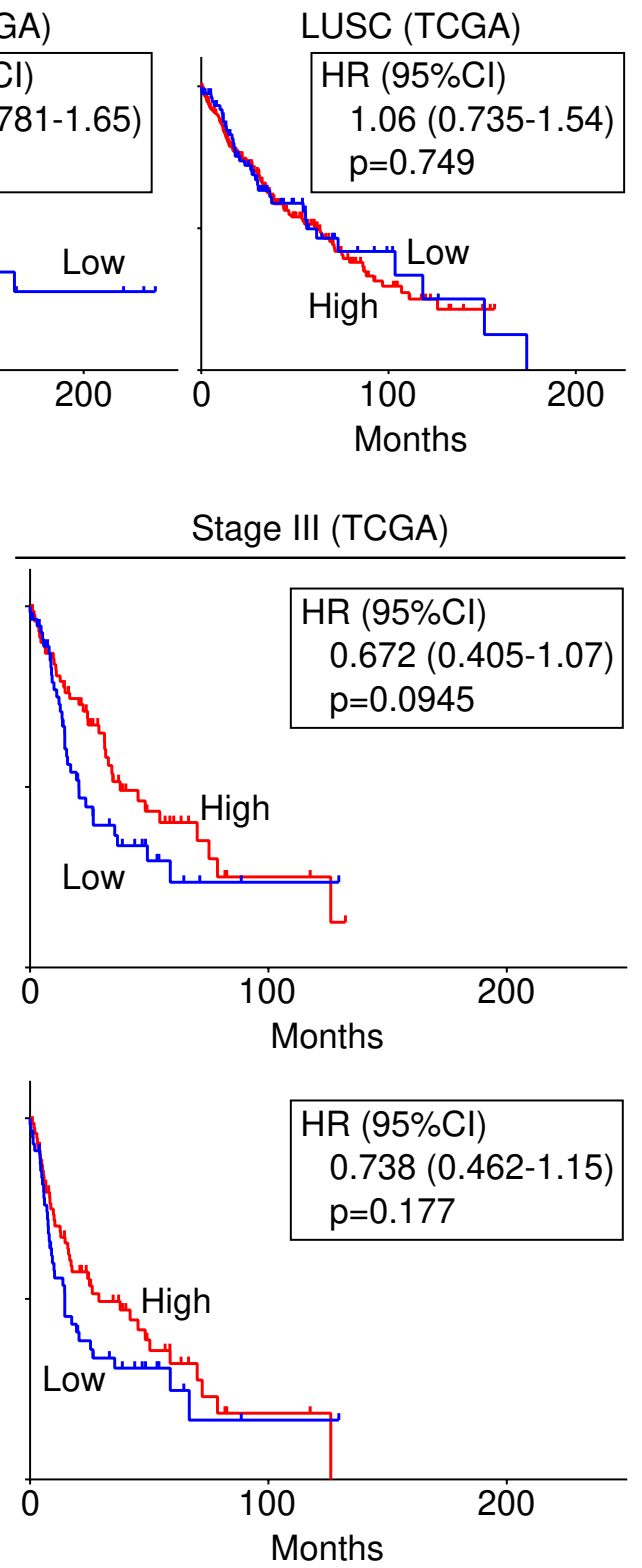

Stage III (TCGA)

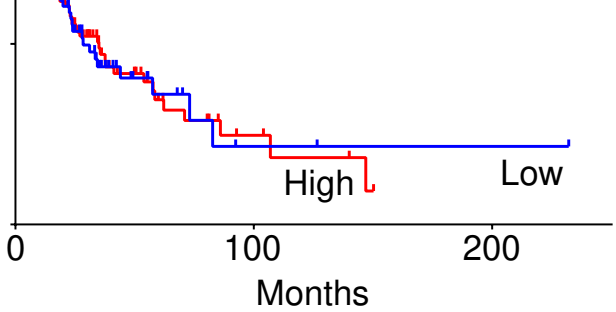


1 Extended Data Fig. 3. Variable effect of Meflin expression on NSCLC patients' outcomes

2 (a, b) Flow diagrams of selection of eligible NSCLC patients (pts) for analysis of OS and DFS in 3 our institution (NUH) (a) or those available in the TCGA database (b).

4 (c) OS of Meflin-high (red) and -low (blue) Stage I-III LUAD or LUSC samples obtained from 5 our institution (NUH, left) or the TCGA database (TCGA, right). The Meflin-high group showed 6 a poor outcome compared to the Meflin-low group in the LUAD cohort of NUH (far left panel).

7 (d) NSCLC samples available in the TCGA database were classified by stage, followed by 8 comparison of the OS (top panels) and PFS (lower panels) of the Meflin-high (red) and -low

9 (blue) groups. Note that the Meflin-high and -low groups' outcomes were comparable in Stage I 10 and II samples (left and middle panels), whereas the Meflin-high group tended to show a

11 favourable outcome compared to the Meflin-low group in Stage III samples, although the 12 difference was not statistically significant (right panel). 
a

Patients with NSCLC who received ICB monotherapy with
nivolumab, pembrolizumab, or atezolizumab at NUH
from 2016 to Aug 2019: 132 pts

No accessibility to specimens: 19 pts

Inappropriate / shortage of specimens: 15 pts

Survival evaluable patients: 98 pts

Response evaluable patients: 94 pts b

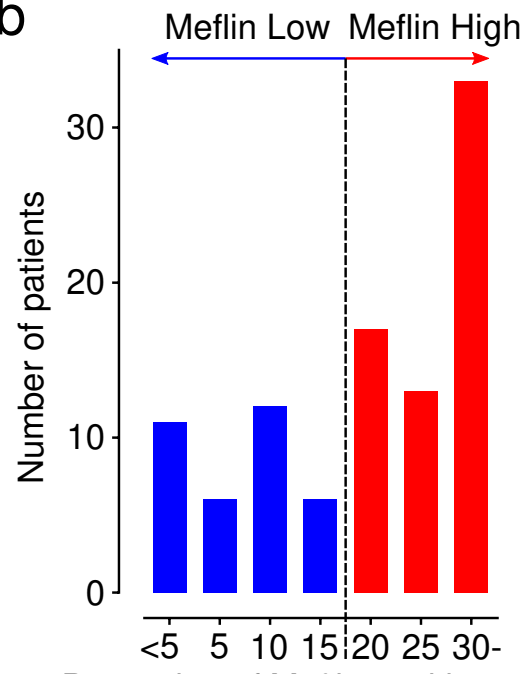

Proportion of Meflin positive cells (\%)

C

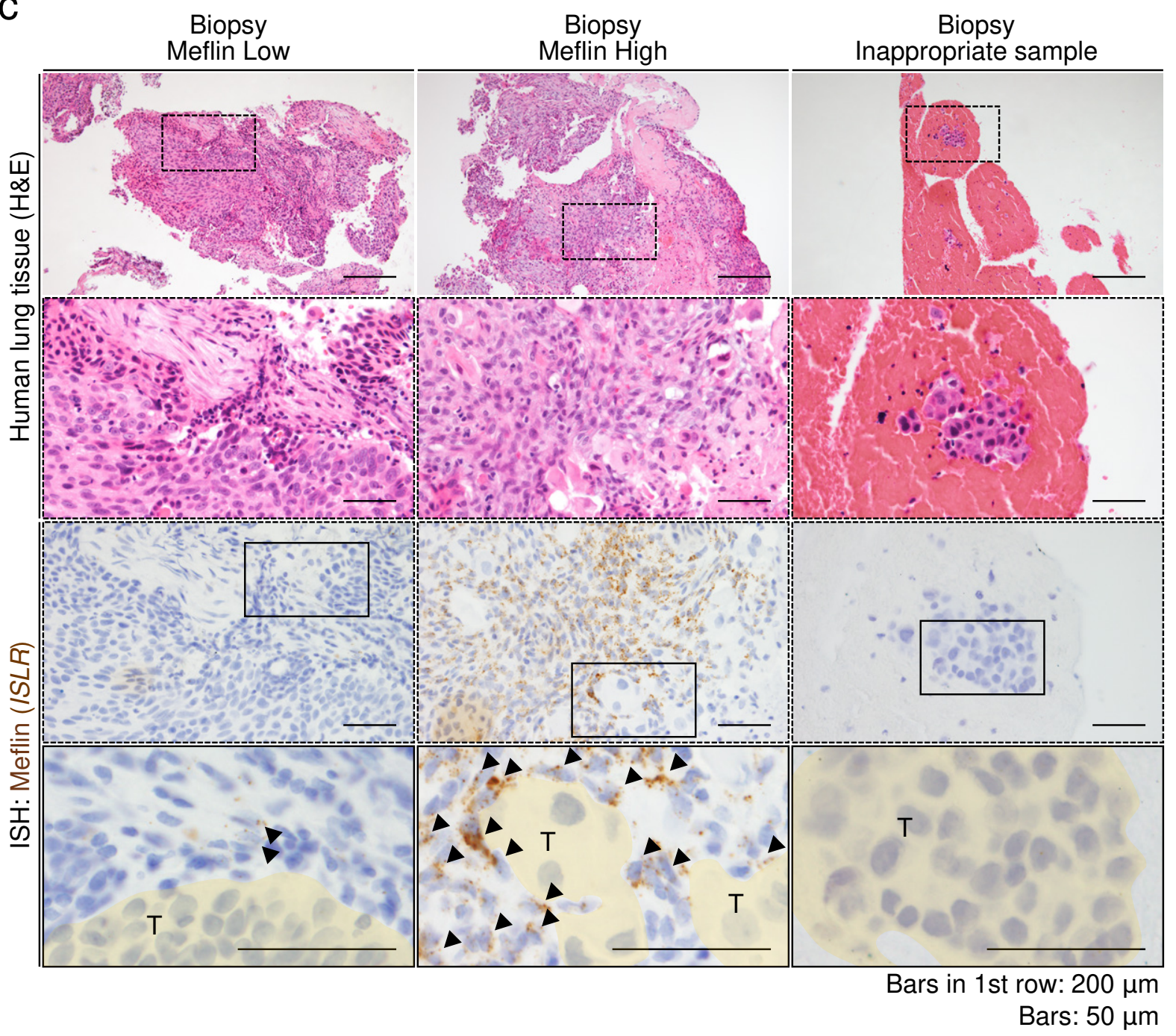

ExFig.4 Miyai et al. 
1 Extended Data Fig. 4. Analysis of Meflin expression in human NSCLC cases treated with 2 immune checkpoint blockade (ICB)

3 (a) Flow diagram of the selection of eligible NSCLC patients who received ICB monotherapy for 4 ORR analysis in NUH.

5 (b) Distribution of NSCLC patients stratified by the proportion of Meflin ${ }^{+}$CAFs determined by $6 \quad$ ISH analysis. The number of Meflin ${ }^{+}$CAFs was counted in randomly selected five high-power 7 microscopic fields. The proportion of Meflin ${ }^{+}$CAFs was calculated as the ratio of Meflin ${ }^{+}$cells 8 to all stromal cells with a spindle morphology. Meflin-high was defined as $\geq 20 \%$ of stromal $9 \quad$ cells stained for Meflin by ISH.

10 (c) Representative images of Meflin-high and -low NSCLC biopsy samples stained with H\&E 11 (upper panels) and Meflin mRNA by ISH (lower panels). The boxed areas are magnified in the 12 lower panels. Areas filled with yellow denote T cells. Arrow heads denote Meflin ${ }^{+}$CAFs in the 13 stroma. The far-right panels indicate an example of biopsy samples that are considered 14 inappropriate for evaluation, in which no stromal cells were found in the sample.

15 
a

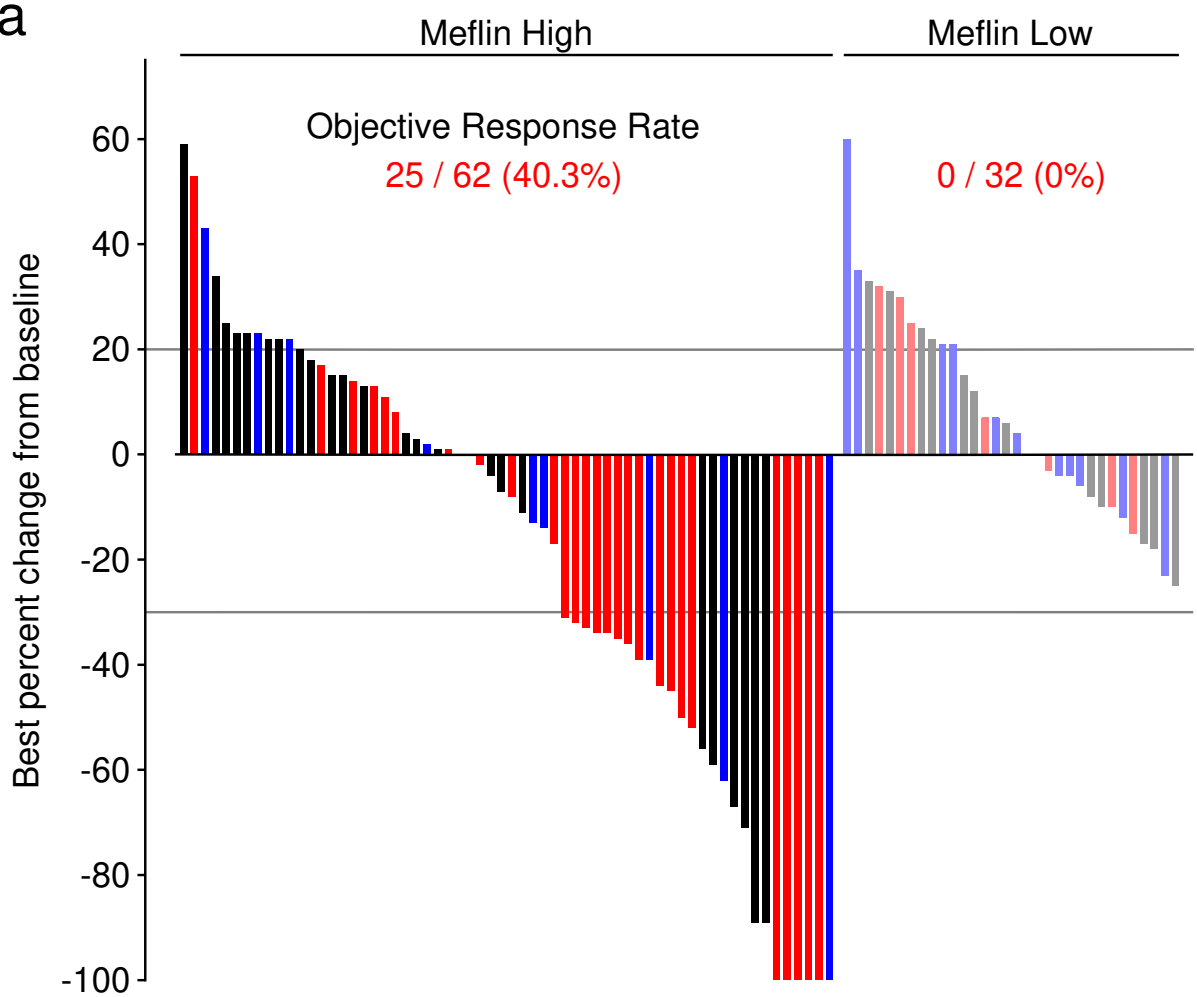

$\mathrm{p}<0.0001$ (Fisher's exact test)

Nivolumab

Pembrolizumab

Atezolizumab

b

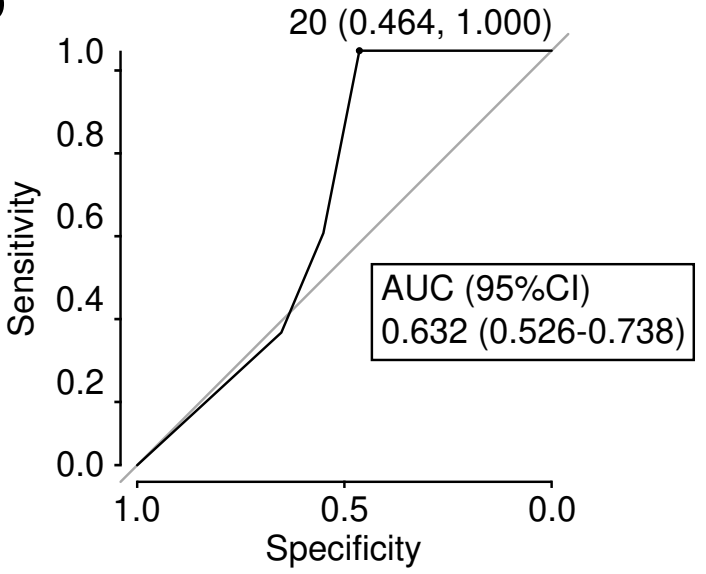

C

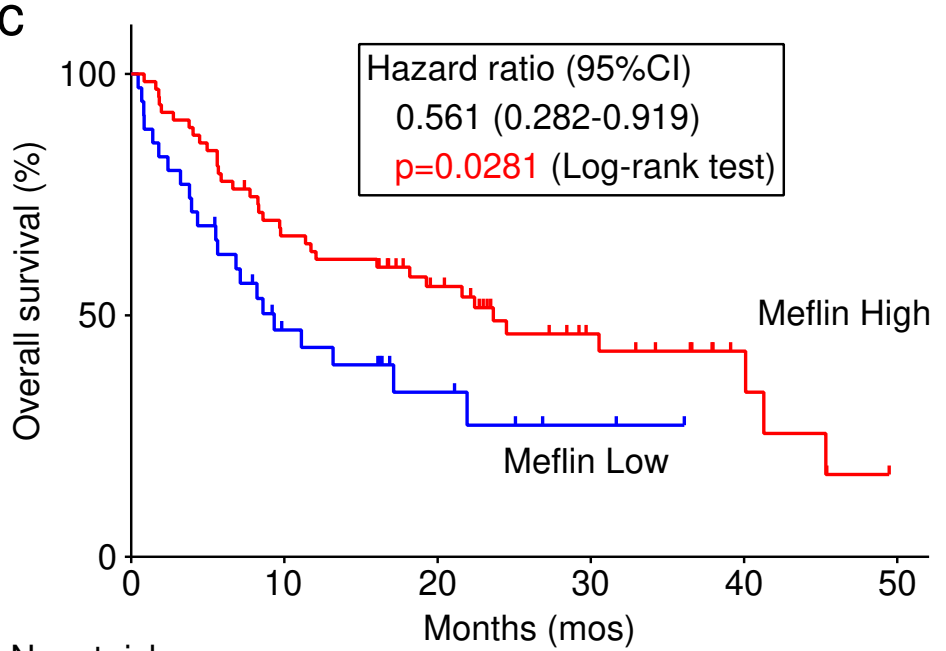

No. at risk

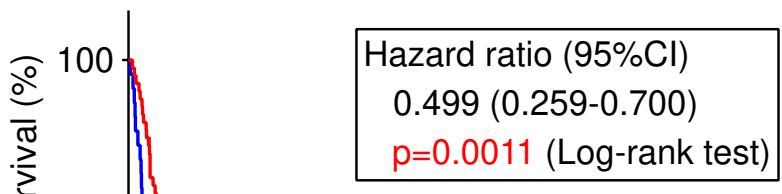

High 63 $41 \quad 27$

$27 \quad 13$

5

$0 \quad 0$ Low 34

No. at risk

Low 35

13

6

2

Meflin

High

$22(62.9 \%)$

No. of events $(\%)$

$35(55.6 \%)$

$9.34(5.52-21.9)$

\begin{tabular}{|lcc|}
\hline Meflin & High & Low \\
No. of events (\%) & $52(83.9 \%)$ & $33(97.1 \%)$ \\
Mos, median (95\%Cl) & $5.29(2.76-9.60)$ & $1.48(1.08-5.10)$ \\
\hline
\end{tabular}

Fig. 3 
Fig. 3. NSCLC patients with high $\mathrm{Meflin}^{+} \mathrm{CAF}$ infiltration exhibit favourable response to ICB therapy

3 (a) A waterfall plot showing changes in tumour size from baseline determined according to

4 iRECIST criteria in Meflin-high (left) and -low (right) NSCLC patients who receive ICB

5 monotherapy. Black, red and blue bars indicate patients treated with nivolumab, pembrolizumab, 6 and atezolizumab, respectively.

7 (b) A receiver operating characteristics (ROC) curve for the percentage of Meflin ${ }^{+}$CAFs in 8 tumour stroma. The ROC curve was obtained by plotting sensitivity against 1 - specificity at 9 each threshold setting. The area under the curve (AUC) $(0.632 ; 95 \%$ CI, $0.526-0.738)$ shown in 10 the plot summarises the performance of Meflin ${ }^{+}$CAFs in tumour stroma.

11 (c) OS (left) and PFS (right) of Meflin-high (red) and -low (blue) NSCLC patients treated with 12 ICB therapy. Meflin-high group showed a favourable response to ICB therapy compared to 13 Meflin-low group. Shown in the boxes below the plots are the observed numbers of events 14 (deaths or disease progression) and median survival (months) of Meflin-high and -low groups 15 over the follow-up periods. Mos, months. 
$a$
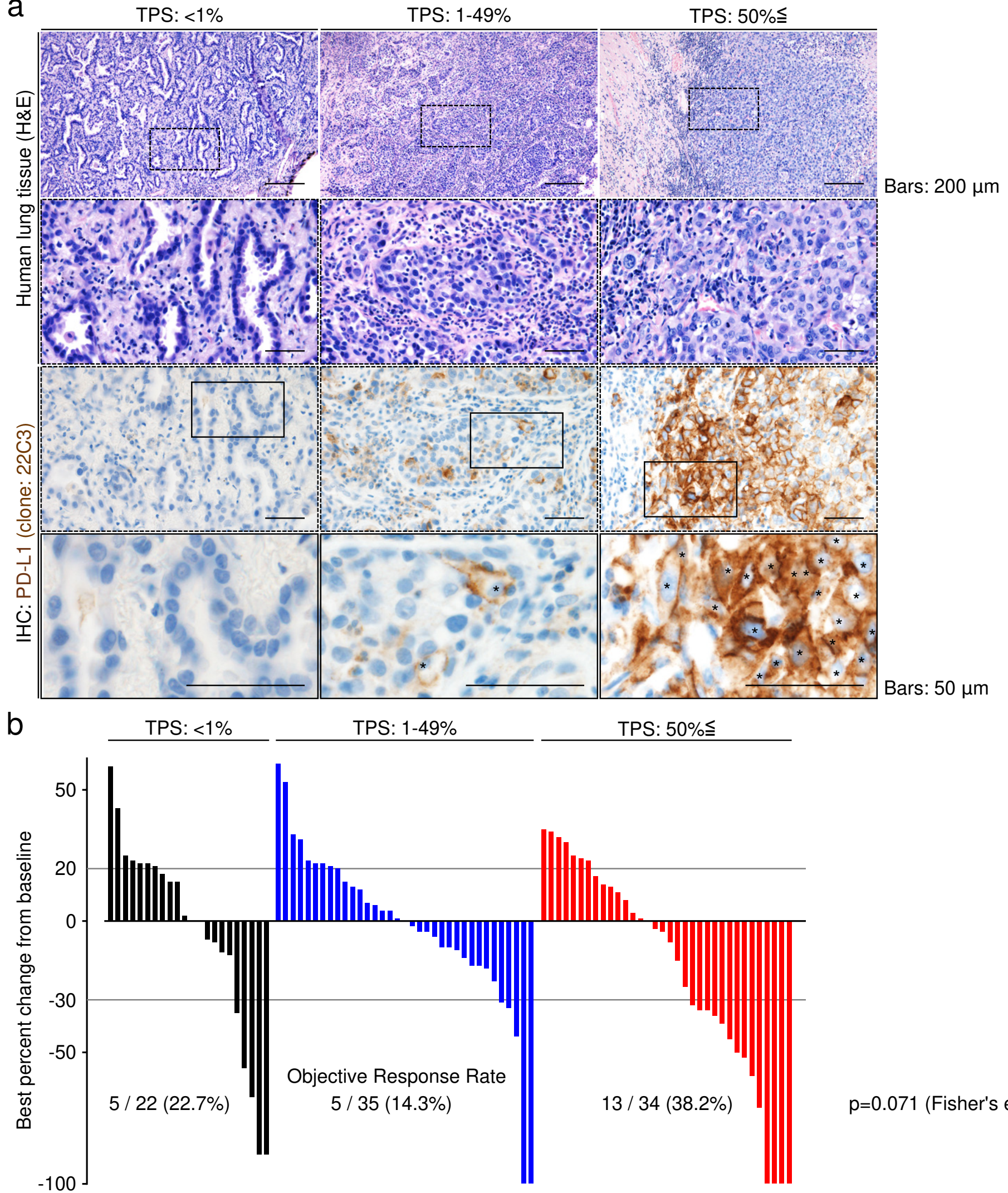

$\mathrm{p}=0.071$ (Fisher's exact test)

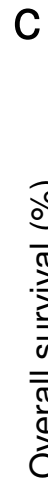

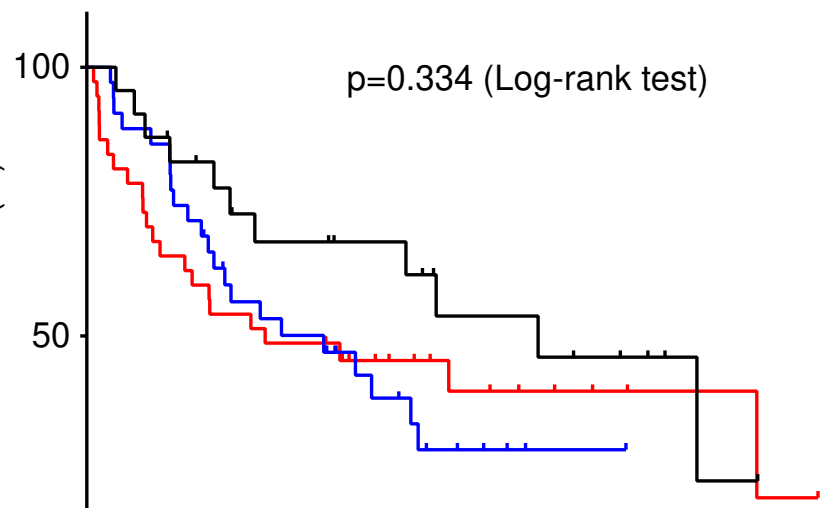

ExFig.5 Miyai et al. Months

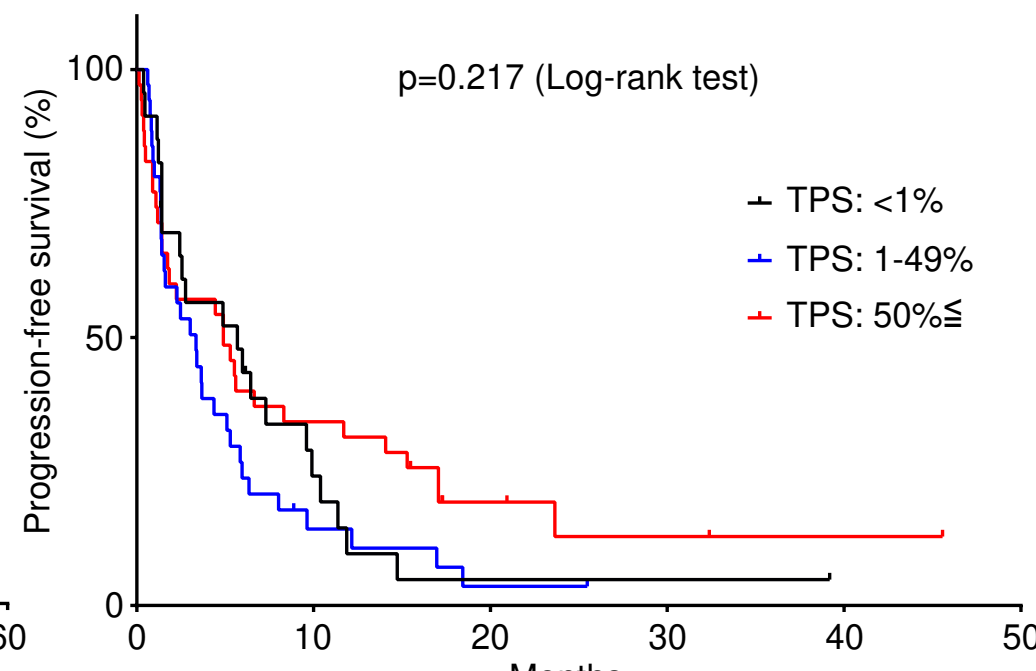


1 Extended Data Fig. 5. No significant correlation between PD-L1 expression in tumour cells

2 and response to ICB therapy in the NSCLC cohort of NUH

3 (a) Tissue sections prepared from resected samples of NSCLC patients who received ICB

4 monotherapy were stained for H\&E (upper panels) and PD-L1 by IHC (lower panels).

5 Representative images of cases with different tumour proportion scores (TPS) are shown. Boxed

6 regions are magnified in the lower panels.

7 (b) A waterfall plot showing tumour size changes from baseline determined according to the

$8 \quad$ iRECIST criteria in NSCLC patients with the indicated TPS.

9 (c) OS (left) and PFS (right) of NSCLC patients treated with ICB therapy using the indicated 10 TPS. 
1 Fig. 4. Meflin expression in CAFs correlates with tumour infiltration of $\mathrm{CD}^{+} \mathbf{T}$ cells and vascular area in NSCLC patients treated with ICB monotherapy

3 (a) Tissue sections prepared from tumours of NSCLC patients who received ICB monotherapy 4 were stained by multiplex immunofluorescence for the indicated TIL markers, nuclei and pan-

5 cytokeratin (Pan-CK), followed by imaging with a multispectral imaging system. Representative 6 images of Meflin-high (left, $n=25$ ) and -low (right, $n=7$ ) cases are shown. Boxed regions were 7 magnified in lower panels. The lower panels' graphs show the quantification of the percentage of $8 \mathrm{CD}^{+}, \mathrm{CD}^{+}$and $\mathrm{CD} 4^{+} \mathrm{FoxP}^{+} \mathrm{T}$ cells in all TILs infiltrated in intra-tumour (parenchyma) and 9 stroma regions of each group. Red dots in the graphs denote the responders to ICB monotherapy.

10 (b) Representative images of tissue sections of Meflin-high (upper panel) and -low (lower panel) 11 tumours stained for CD31. The graph on the right shows the quantification of tumour vessel 12 areas in each group following the method described in Extended Data Fig. 7. High magnification 13 views randomly selected from 25 Meflin-high and 7 Meflin-low cases were analysed and 14 quantified. Red dots in the graphs denote the responders to ICB monotherapy. 
1 Extended Data Fig. 6. Quantification of TILs in Meflin-high and -low cases of NSCLC

2 tumours treated with ICB monotherapy

3 Two alternate examples (upper and lower images) of Meflin-high (left) or -low (right) NSCLC

4 tumour sections stained by multiplex immunofluorescence for the indicated TIL markers, nuclei,

5 and pan-cytokeratin (Pan-CK), followed by imaging with a multispectral imaging system. Boxed

6 regions are magnified in the lower panels. The lowest panel graphs show the quantification of the

7 percentage of $\mathrm{CD}^{+} \mathrm{CD}^{+} 5 \mathrm{RO}^{+} \mathrm{T}$ cells, $\mathrm{CD}^{+} \mathrm{CD} 45 \mathrm{RO}^{+} \mathrm{T}$ cells, and $\mathrm{CD} 20^{+} \mathrm{B}$ cells in all TILs

8 infiltrated in the intra-tumour (tumour parenchyma) and stroma regions of Meflin-high $(\mathrm{n}=25)$

9 and -low $(n=7)$. Red dots in the graphs denote responders to ICB monotherapy. 


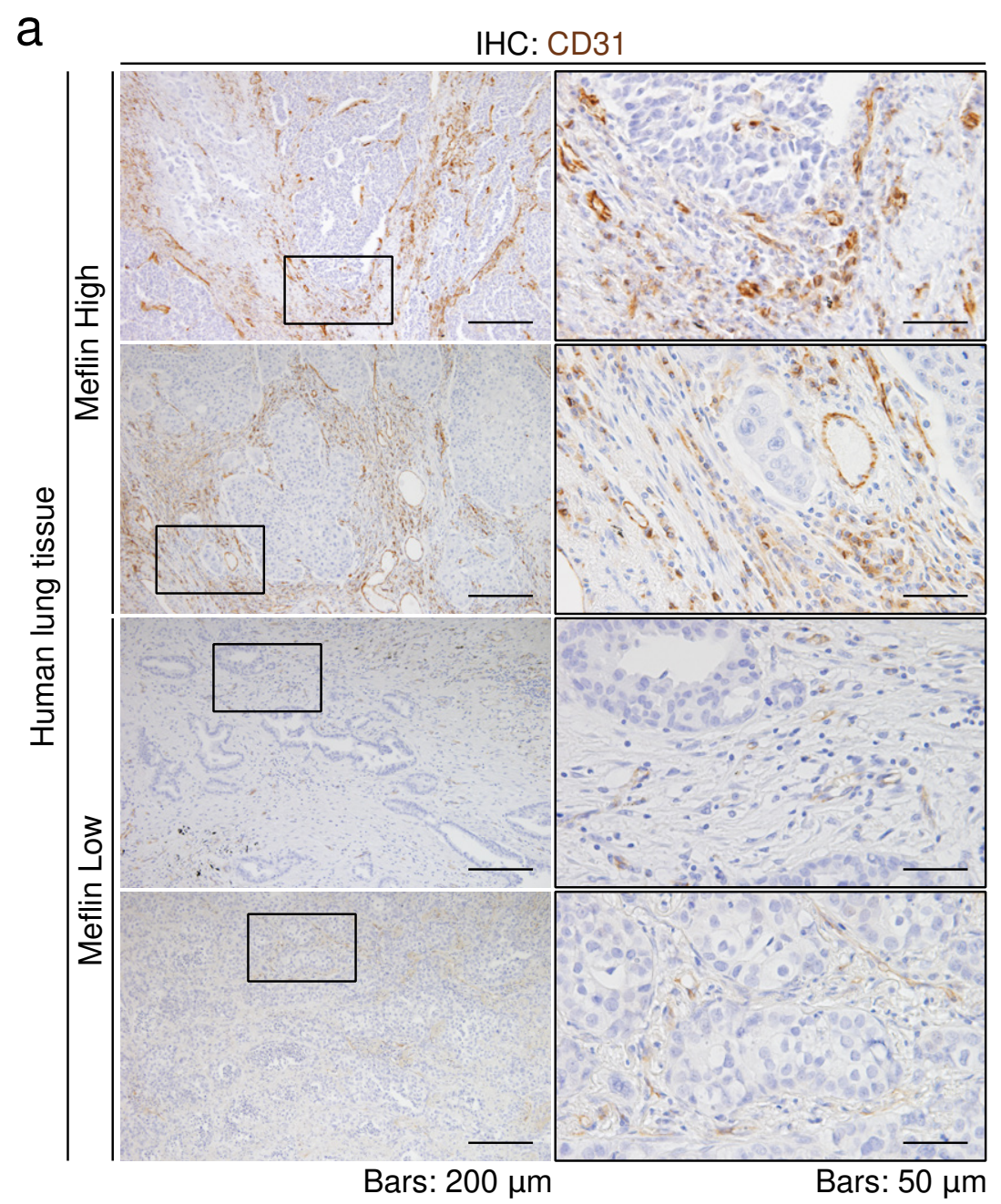

b
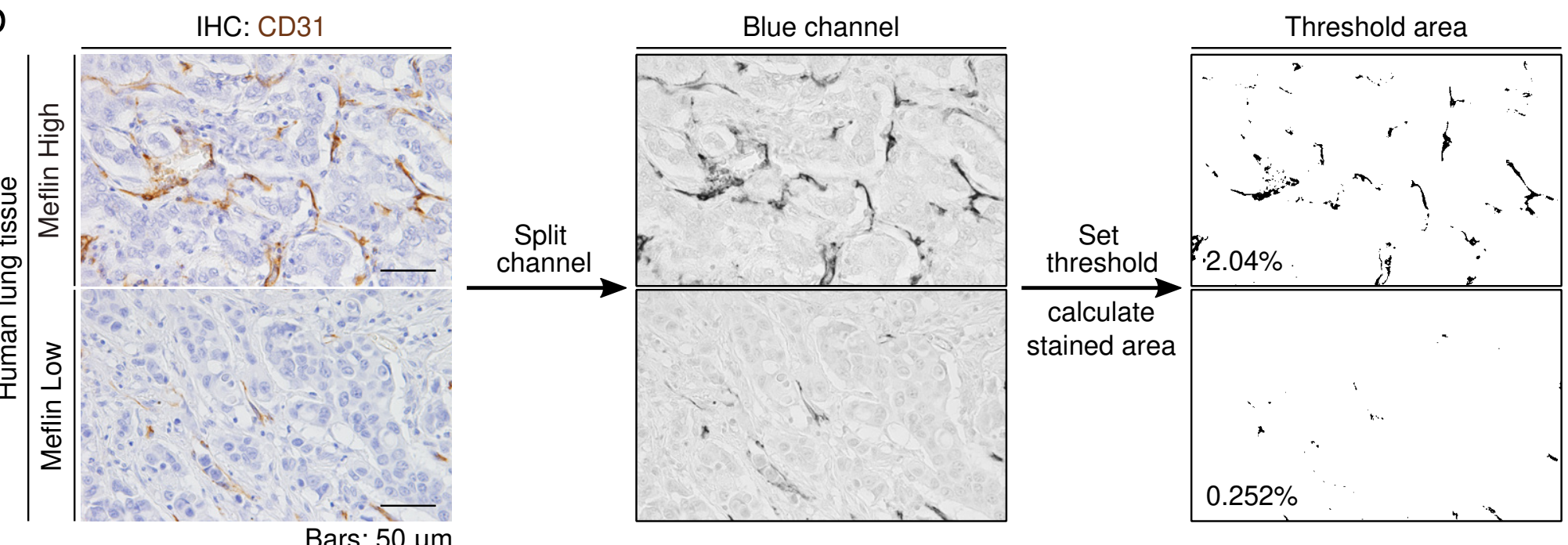

ExFig.7 Miyai et al. 
1 Extended Data Fig. 7. Meflin expression in CAFs correlates with tumour vascular area in

2 NSCLC patients treated with ICB monotherapy

3 (a) Examples of Meflin-high (left) or -low (right) NSCLC tumour sections stained for CD31 to

4 examine tumour vessel areas. The boxed regions are magnified in adjacent panels.

5 (b) Two examples of images used to quantify CD31-positive tumour vessel areas in tumour 6 sections. The brown colour that denotes the CD31-stained regions was extracted using ImageJ

7 software (middle panel), followed by setting a threshold to binarize the images (right panel) and 8 quantify the stained areas. 


\section{$a$}

6-week old female C57BL/6J

(wild-type, W) or

Meflin-KO (K)

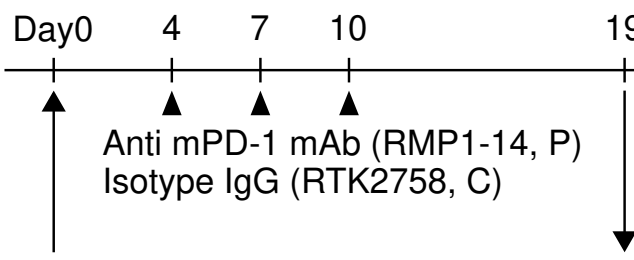

$\stackrel{\text { Survival }}{\longrightarrow}$ analysis

MC-38 (CRC, subcutaneously) Tumour growth $1.0 \times 10^{6}$ cells/body

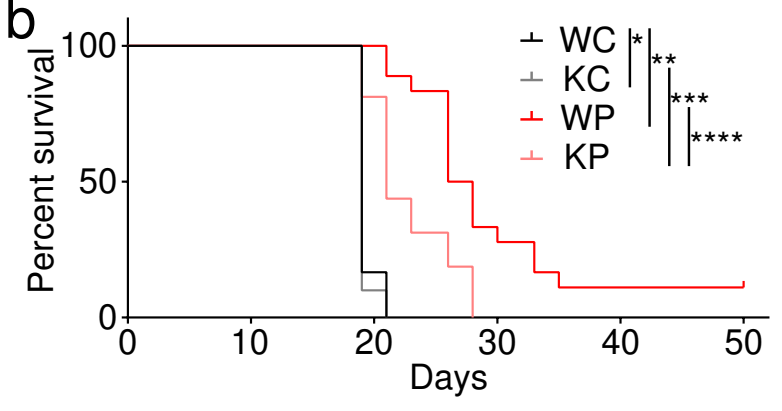

KP

C

WC
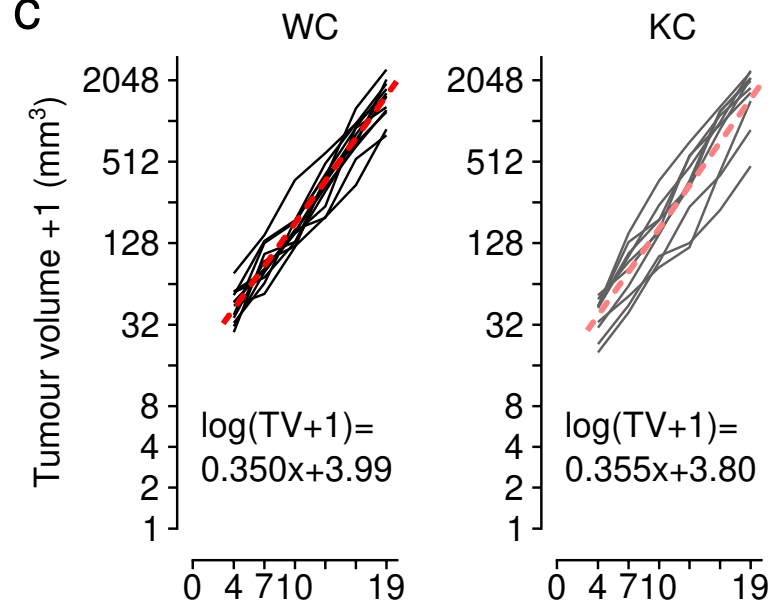

WP
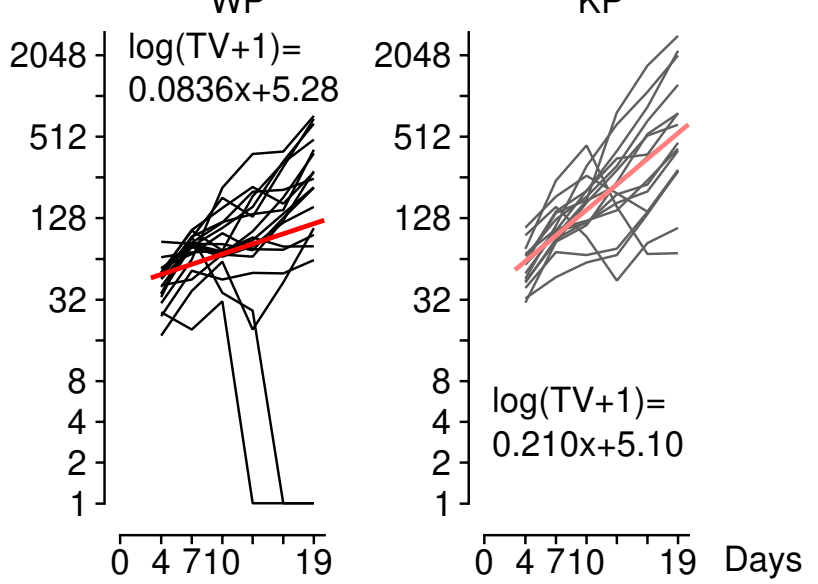

Estimate $(95 \% \mathrm{Cl})$ p-value

\begin{tabular}{lcc}
\hline Time & $0.350(0.283-0.417)$ & $<0.0001$ \\
Time $\times \mathrm{G}$ & $0.0054(-0.0785-0.0888)$ & 0.901 \\
Time $\times$ T & $-0.266(-0.346-0.187)$ & $<0.0001$ \\
Time $\times$ G $\times$ T & $0.121(0.0434-0.198)$ & 0.0041 \\
\hline
\end{tabular}

G: Genotype (wild-type (W) or Meflin-KO (K)) $\mathrm{T}$ : Treatment (control $(\mathrm{C})$ or mPD-1 $(\mathrm{P})$ )

e

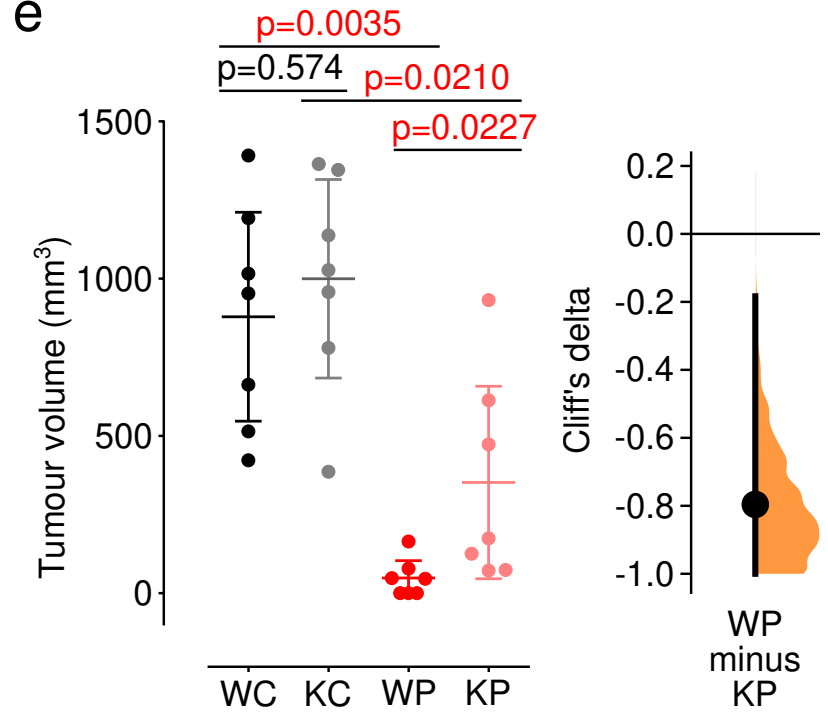

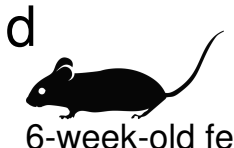
C57BL/6J (W) or Meflin-KO (K)

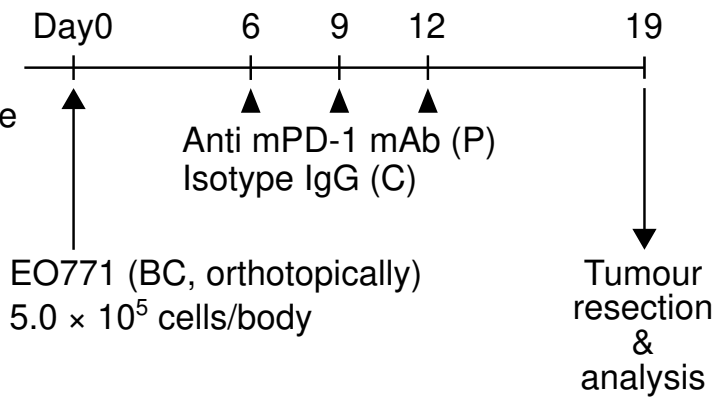

f
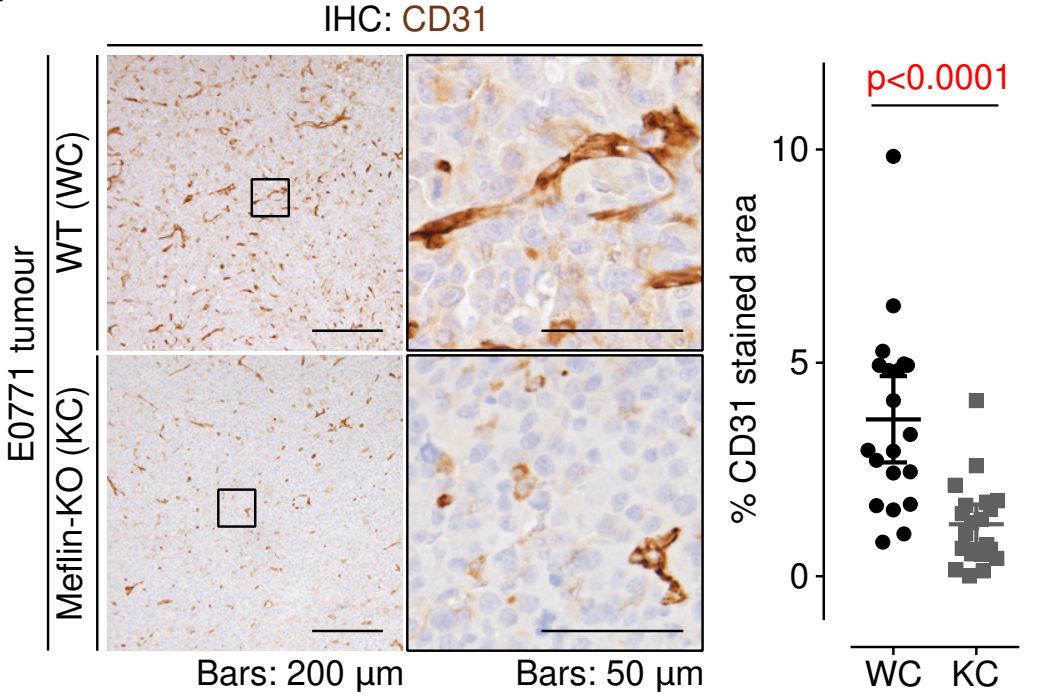

Fig. 5 
(a) An experimental protocol to test the anti-tumour effect of anti-mPD-1 therapy in mice. MC38 mouse CRC cells were transplanted into C57BL/6 wild-type (W) or Meflin-KO (K) mice at day 0 , followed by the intraperitoneal administration of anti-mPD-1 (P) or an isotype control antibody (C).

(b) Survival of wild-type (W) and Meflin-KO (K) mice non-treated (WC and KC, respectively) or treated with anti-PD-1 antibody (WP and KP, respectively). The numbers of mice tested for WC, KC, WP and KP groups were 12, 10, 18 and 16, respectively. *, $\mathrm{p}=0.658 ;{ }^{* *}, \mathrm{p}<0.0001$;

$9 \quad * * *, \mathrm{p}=0.0033 ; * * * *, \mathrm{p}=0.0033$

10 (c) Time courses of the volumes of tumours developed in the indicated groups (black and grey 11 lines). For the log transformation of tumour volumes, one was added to every tumour volume. 12 Red lines indicate linear approximations. The table shown under the graphs shows the restricted 13 maximum likelihood estimates of each parameter in a linear mixed-effects model that includes 14 the interactions of time, time and mouse genetic background $(\mathrm{G})$, time and anti-mPD-1 therapy 15 (T), and time and $\mathrm{G}$ and $\mathrm{T}$, while adding variable effects to the slope and intercept for each 16 individual.

17 (d, e) EO771 mouse BC cells were transplanted into wild-type (W) or Meflin-KO (K) mice at 18 day 0 , followed by the intraperitoneal administration of anti-mPD-1 antibody (P) or an isotype 19 control antibody (C). The number of mice tested for each group was 7. Shown in (e) are the quantification of tumour volume of each group (left) and the non-parametric estimate of effect

21 size calculated by Cliff's delta (right). (f) Tissue sections from EO771 tumours developed in wild-type (WC) and Meflin-KO (KC) were stained for CD31 to visualise tumour vessels (left), followed by the quantification of the 24 stained areas (right). Boxed regions were magnified in adjacent panels. 

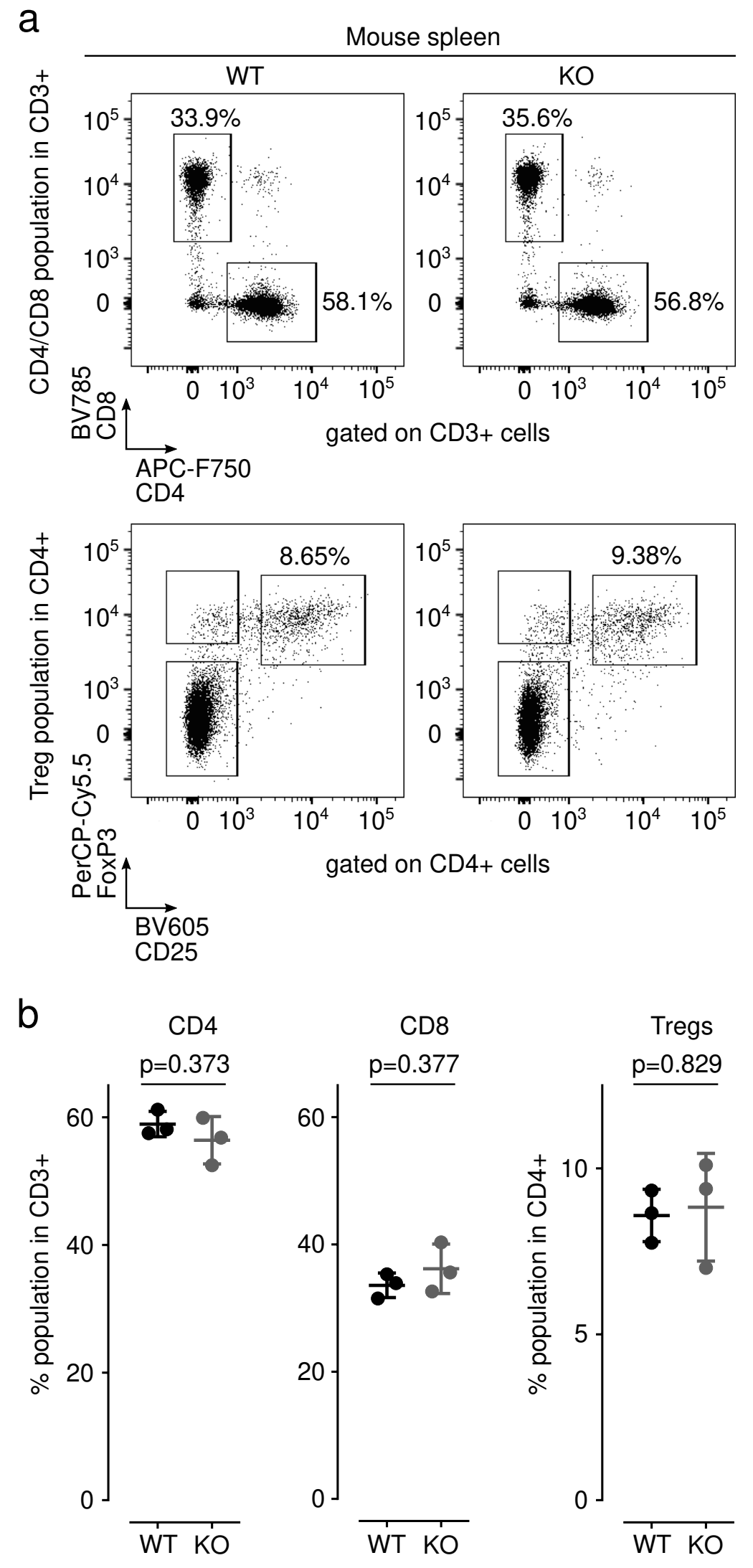

ExFig.8 Miyai et al. 
1 Extended Data Fig. 8. Analysis of $\mathbf{T}$ cells in the spleen of wild-type or Meflin-KO mice

(a) $\mathrm{CD}^{+} \mathrm{T}$ cells were first gated among splenocytes isolated from the spleens of wild-type (W)

3 or Meflin-KO $(\mathrm{K})$ mice by positive selection, and then these cells were analysed for expression

4 of the indicated T cell markers by flow cytometric (FCM) analysis.

5 (b) Quantification of $\mathrm{CD}^{+}$and $\mathrm{CD}^{+} \mathrm{T}$ cells and $\mathrm{CD} 4^{+} \mathrm{CD} 25^{+} \mathrm{FoxP} 3+$ regulatory $\mathrm{T}$ cells (Tregs)

6 in spleens isolated from WT and $\mathrm{KO}$ mice. 
a

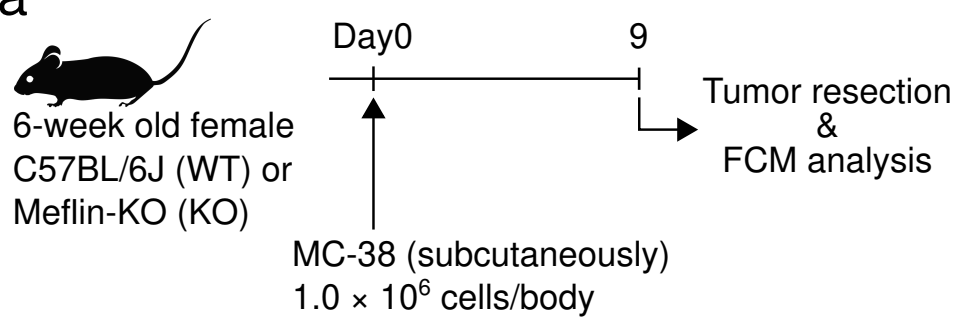

b

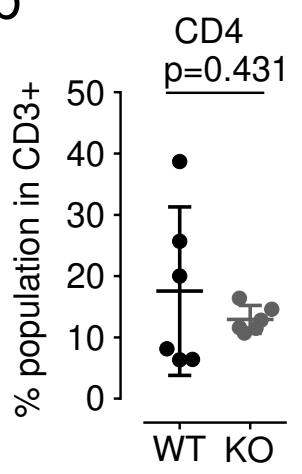

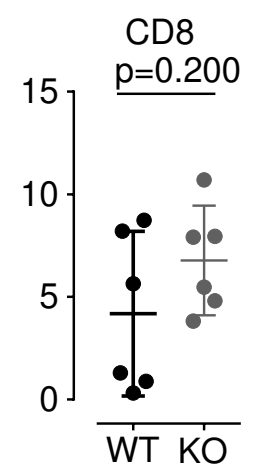

C

$\mathrm{CD}^{+}$cells (CD4+ $4^{+} \mathrm{CD} 25^{-}$FoxP3 ${ }^{-}$cells)

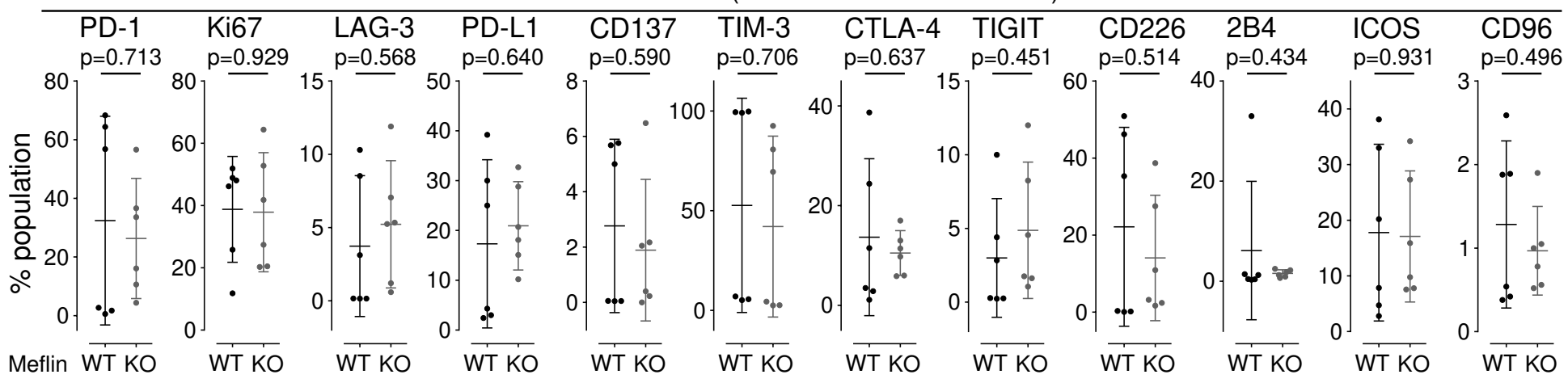

d $\mathrm{CD8}^{+}$cells

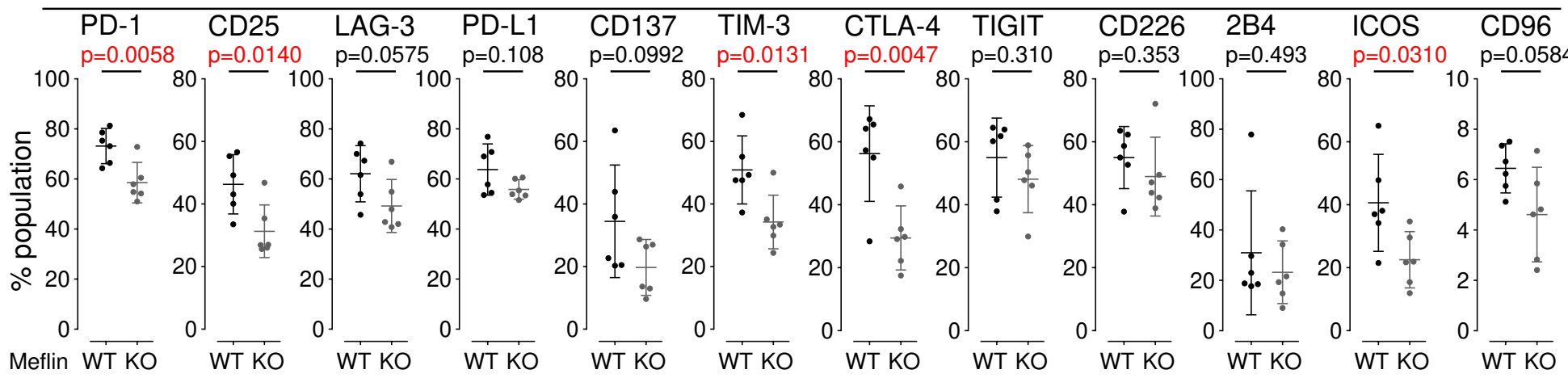

e Tregs (CD4 ${ }^{+} \mathrm{CD}_{25} \mathrm{FoxP}^{+}$cells)

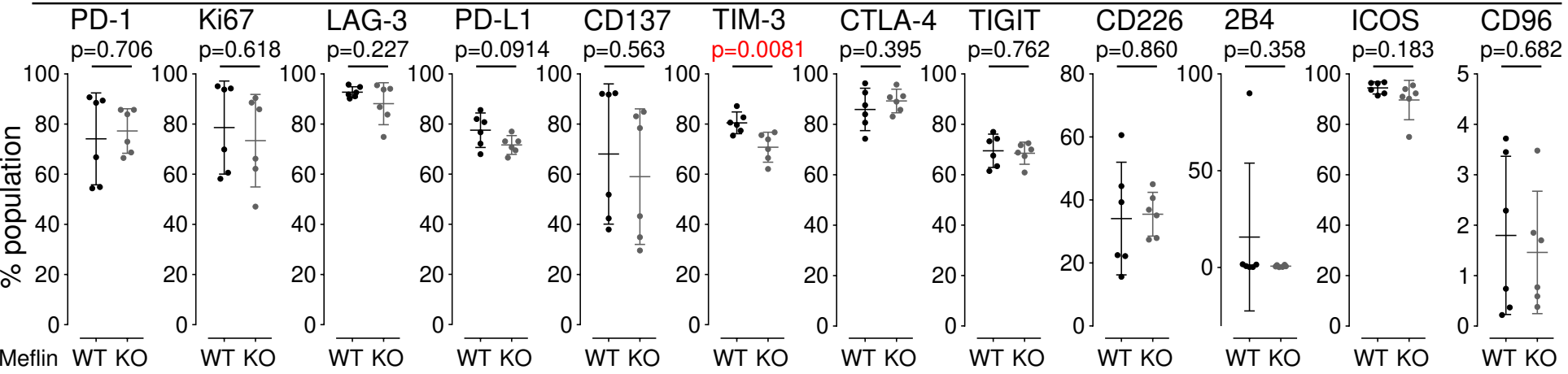

ExFig.9 Miyai et al. 
1 Extended Data Fig. 9. Analysis of the expression of various markers on TILs isolated from

2 tumours developed in wild-type or Meflin-KO mice

3 (a, b) MC-38 cells were transplanted into wild-type (WT) or Meflin-KO (KO) mice on day 0,

4 followed by tumour resection and flow cytometric (FCM) analysis on day 9 (a). For FCM

5 analysis, $\mathrm{CD}^{+} \mathrm{T}$ cells were first gated among TILs isolated from the tumours by positive

6 selection, and then these cells were analysed for expression of the indicated $\mathrm{T}$ cell markers. The

7 graphs on the right show the quantification of $\mathrm{CD} 4^{+}$and $\mathrm{CD} 8^{+} \mathrm{T}$ cells and $\mathrm{CD} 4^{+} \mathrm{CD} 25^{+} \mathrm{FoxP} 3^{+}$

8 regulatory T cells (Treg) in TILs isolated from tumours developed in WT and KO mice.

9 (c-e) $\mathrm{CD}^{+}{ }^{+} \mathrm{CD} 4^{+} \mathrm{CD} 25^{-}{ }^{-}$oxP $33^{-}, \mathrm{CD}^{+}{ }^{+} \mathrm{CD} 8^{+}$, and $\mathrm{CD}^{+}{ }^{+} \mathrm{CD} 25^{+} \mathrm{FoxP}^{+} \mathrm{T}$ cells sorted from TILs

10 isolated from tumours developed in wild-type (WT) or Meflin-KO (KO) mice were analysed for

11 the indicated markers by FCM analysis.

12 
Wild type allele

Meflin-Cre

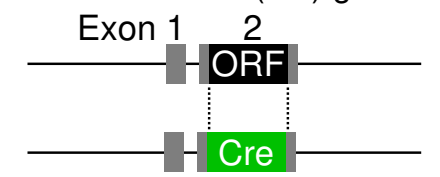

$+$

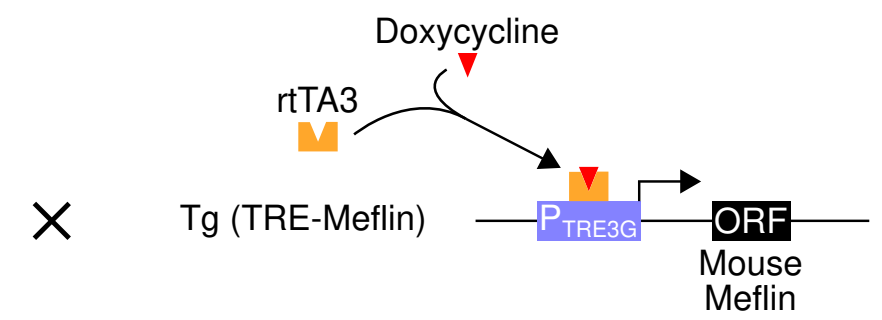

Rosa26-LSL-rtTA3 -CAG-4Stop-rtTA3 LoxP LoxP

b

Meflin-Cre ${ }^{+/+}$

R26-LSL-rtTA3 ${ }^{+/-}$

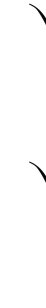

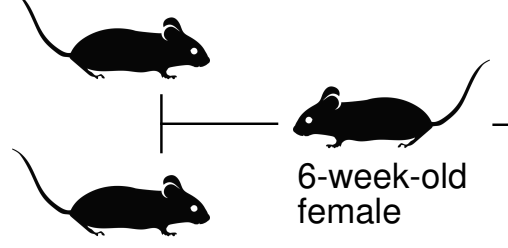

$\operatorname{Tg}$ (TRE-Meflin) female<smiles>C1CCCC1</smiles>

MC-38 (subcutaneously) $1.0 \times 10^{6}$ cells $/$ body

Fed with $5 \%$ sucrose water containing doxycycline $(2 \mathrm{mg} / \mathrm{mL})$

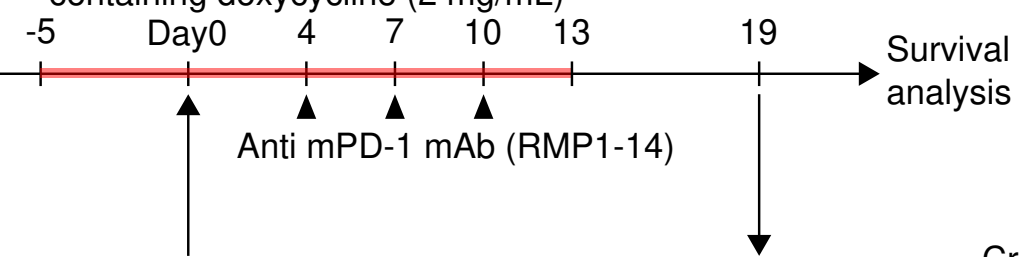

Tumor growth analysis e

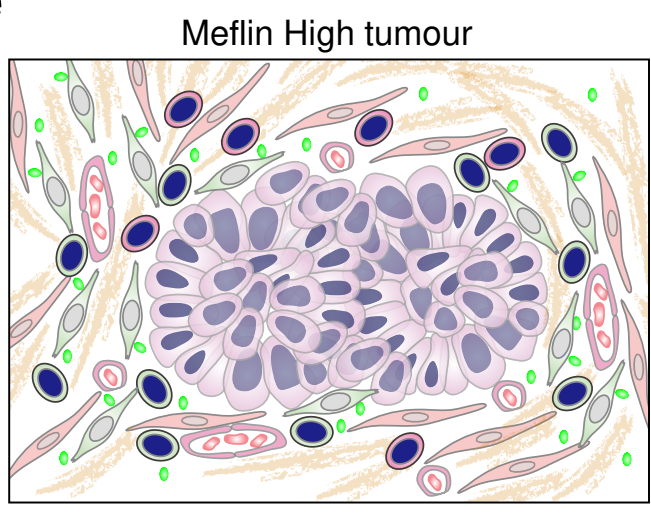

- Control $(\mathrm{n}=8)$

- TO $(n=5)$

p-value (log-rank test) 0.0026
C$$
\text { e }
$$

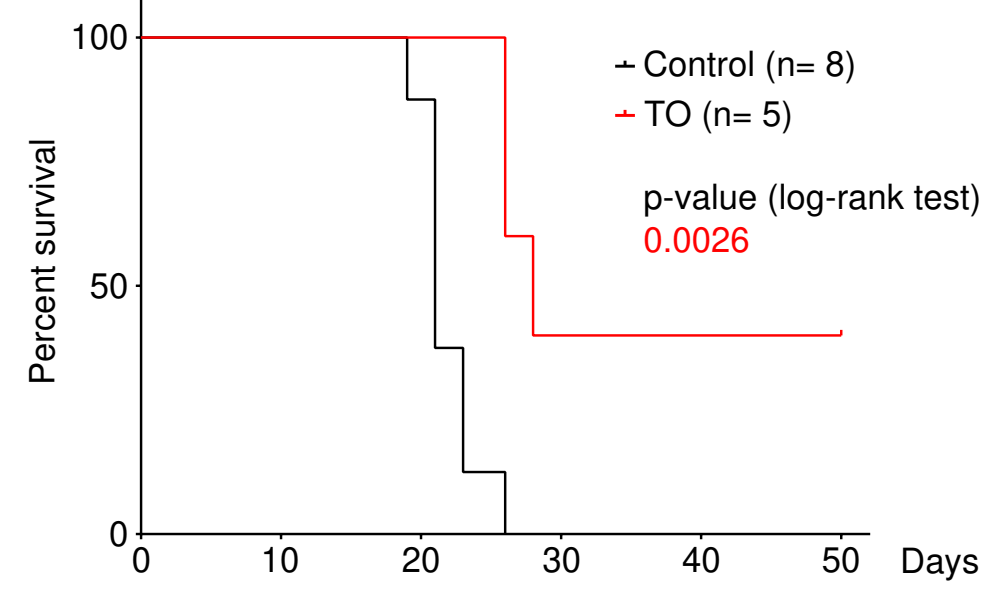

$\mathrm{Cre}^{+}, \mathrm{rtTA3}^{+}, \mathrm{Tg}^{+}:$Meflin-TO (TO) $\mathrm{Cre}^{+}$, rtTA3$^{-}, \mathrm{Tg}^{+}$: Control

d
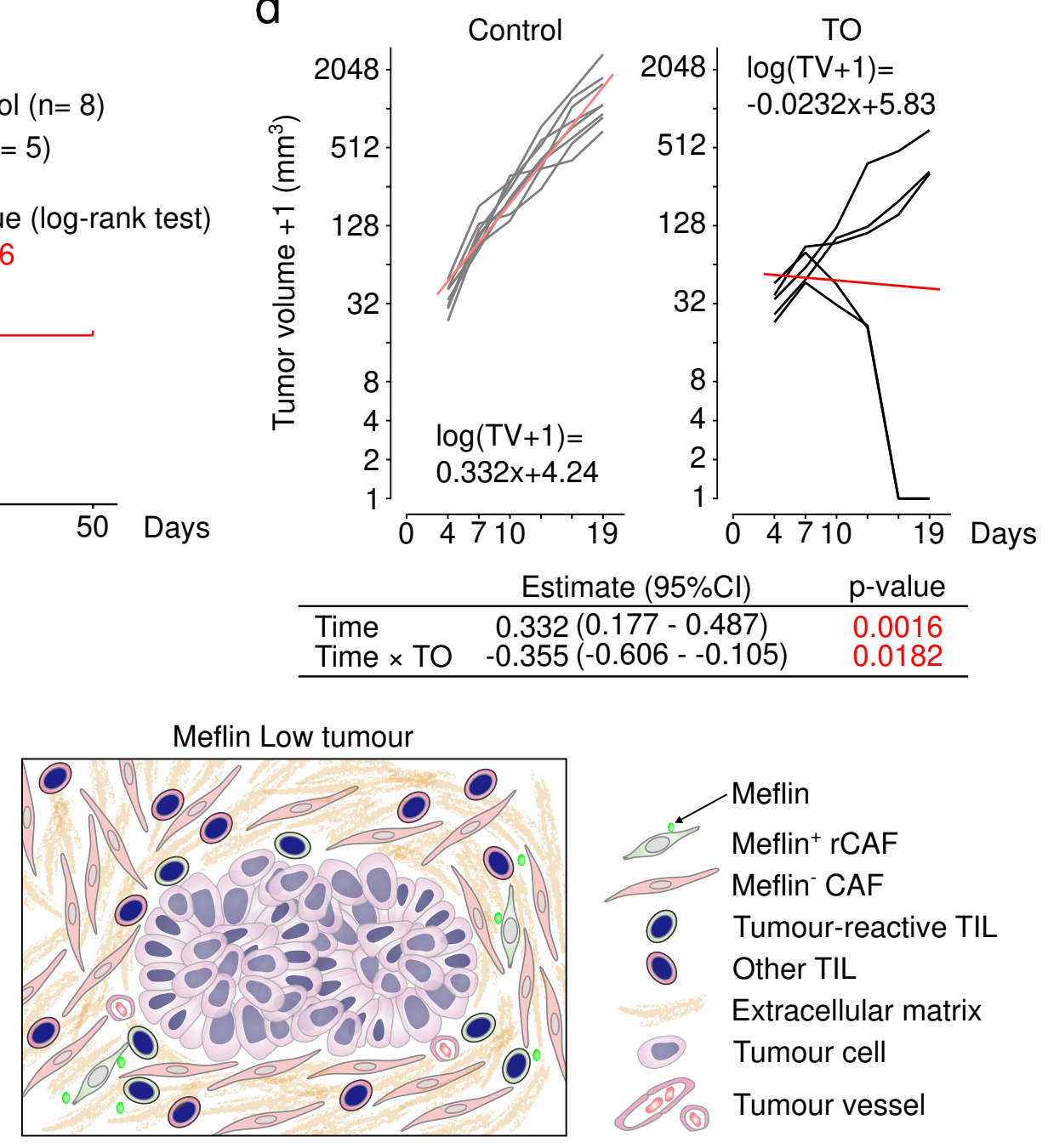

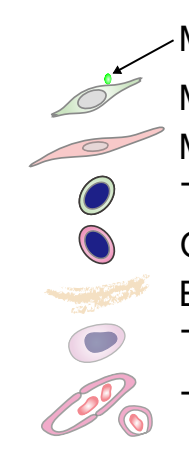

Meflin

Meflin- CAF

Tumour-reactive TIL

Other TIL

Extracellular matrix

Tumour cell

Tumour vessel
Meflin ${ }^{+}$rCAF

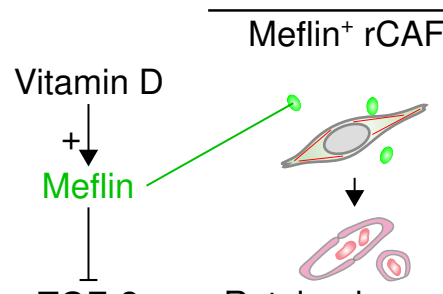

TGF- $\beta \quad$ Retained vessels Collasped vessels signaling

Fig. 6

Treatment success Treatment failure 
(a) A diagram of the generation of the mouse line carrying Meflin-Cre, which constitutively expresses Cre under the control of the Meflin promoter, Rosa26-LSL-rtTA3, and Tg (TREMeflin) alleles that exhibit induced Meflin expression in Meflin-lineage cells upon doxycycline administration. ORF; open reading frame, CAG; chicken 3 -actin promoter, Stop; stop element, rtTA3; the third-generation reverse tetracycline-regulated transactivator, Tg; transgenic, TRE; tetracycline-response element.

9 (b) Meflin-Cre ${ }^{+/+}$; Rosa26-LSL-rtTA3 ${ }^{+/-}$mice were crossed with TRE-Meflin transgenic mice.

10 The resultant 6-week-old female mice fed with doxycycline were subcutaneously implanted with

11 MC-38 cells, followed by anti-mPD-1 therapy, genotyping, and tumour analysis. Mice that

12 harbour all of the Meflin-Cre, Rosa26-LSL-rtTA3 and TRE-Meflin alleles were termed Meflin

13 TO (Tet-on) mice.

(c) Survival of control (Meflin-Cre ${ }^{+/-}$; Rosa26-LSL-rtTA3 ${ }^{-/}$; TRE-Meflin) and Meflin TO

15 (Meflin-Cre ${ }^{+/}$; Rosa26-LSL-rtTA3 ${ }^{+/-}$; TRE-Meflin) mice treated with anti-mPD-1 antibody.

16 (d) Time courses of the volumes of tumours developed in the indicated groups (black and grey

17 lines). For the log transformation of tumour volumes, one was added to every tumour volume.

18 Red lines indicate linear approximations. The table shown under the graphs shows the restricted

19 maximum likelihood estimates of each parameter in a linear mixed-effects model that includes

20 the interactions of time and time and induced Meflin expression (TO), while adding variable

21 effects to the slope and intercept for each individual.

22 (e) Graphical summary and working hypothesis for CAF heterogeneity and its role in ICB 23 response. Our data demonstrated that infiltration of $\mathrm{Meflin}^{+} \mathrm{CAFs}$ was associated with perfused 
1 tumour vessels, which may allow blood and immune cells and macromolecules like antibodies to 2 access the tumour.

3

4 
a

Meflin-Cre ${ }^{+/-}$

R26-LSL-rtTA3 ${ }^{+/+}$

Fed with $5 \%$ sucrose water

with (red) or without (blue)

doxycycline (DOXY; $2 \mathrm{mg} / \mathrm{mL}$ )
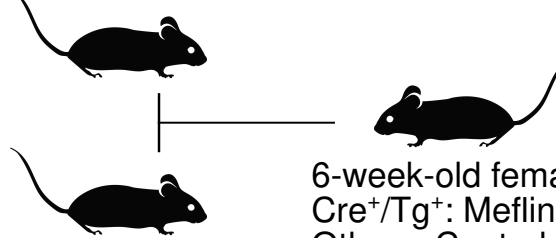

-5 day 0

6 -week-old female $\mathrm{Cre}^{+} / \mathrm{Tg}^{+}$: Meflin-TO (TO)

Tg (TRE-Meflin)

( $\mathrm{Tg})$ Others: Control (C)

MC-38 (subcutaneously) $1.0 \times 10^{6}$ cells/body EO771 (orthotopically) $5.0 \times 10^{5}$ cells/body

b
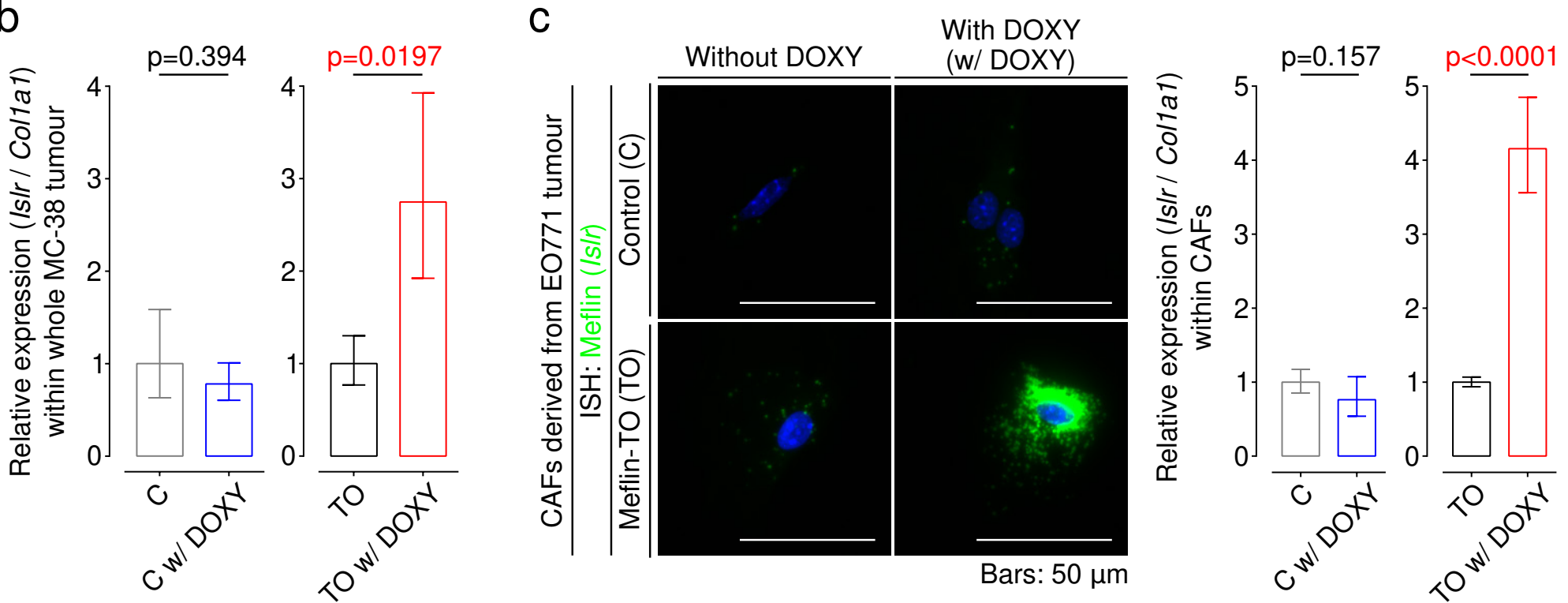

d
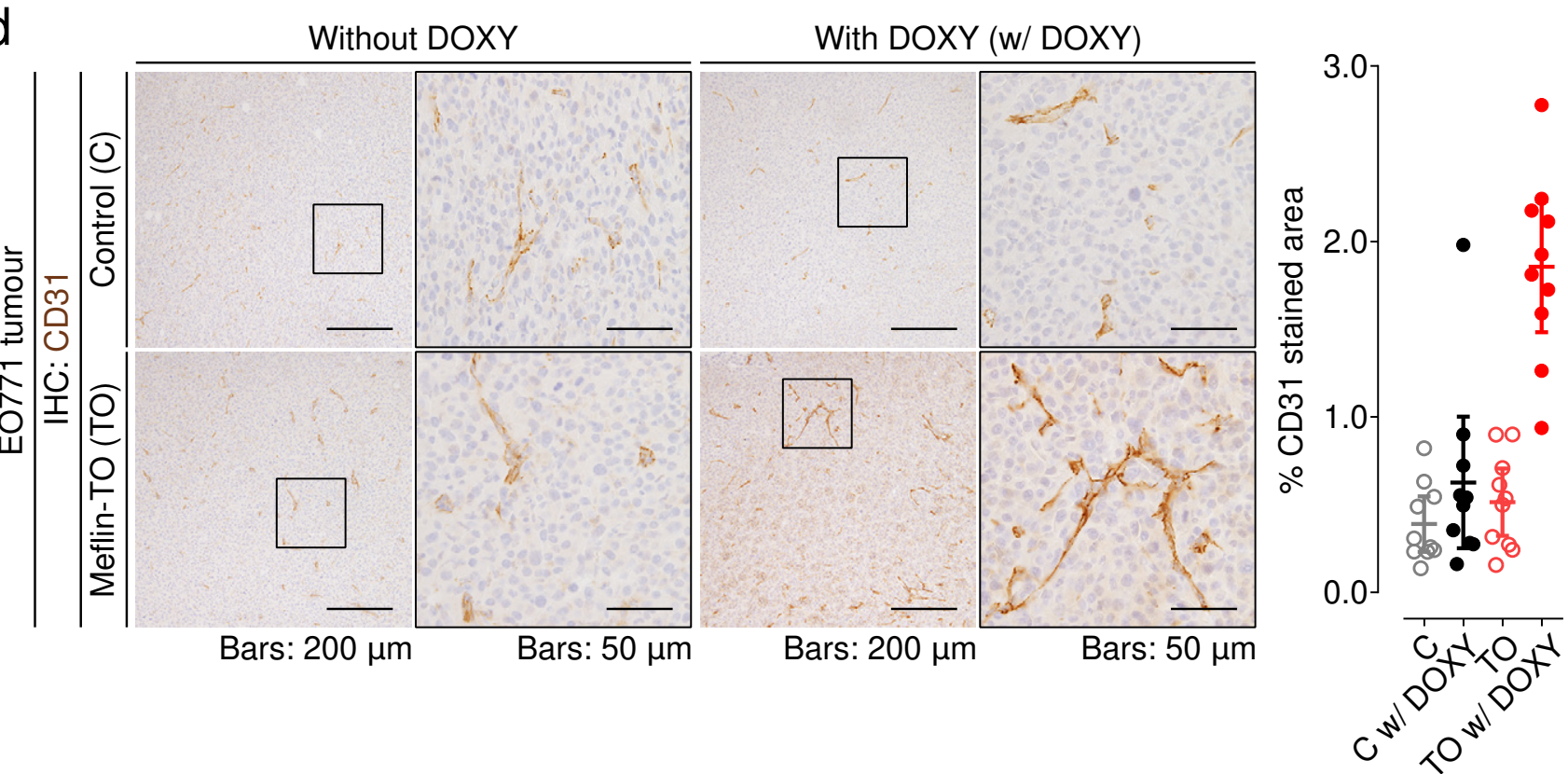

ExFig.10 Miyai et al. 

response to anti-mPD-1 therapy in mice

(a) Meflin-Cre ${ }^{+/+}$; Rosa26-LSL-rtTA3 ${ }^{+/-}$mice were crossed with TRE-Meflin transgenic (Tg) mice. The resultant 6-week-old female mice were fed with or without doxycycline (DOXY) supplementation, and subcutaneously or orthotopically implanted with MC-38 or EO771 cells, respectively, followed by the analysis of the developed tumours and isolation of CAFs. Mice that harboured all of the Meflin-Cre, Rosa26-LSL-rtTA3, and TRE-Meflin alleles were termed Meflin-TO (Tet-on) mice.

(b) Total RNA extracted from whole tumours of control (C) (Meflin-Cre-/-; Rosa26-LSL-rtTA3 ${ }^{+/-}$; TRE-Meflin) and Meflin TO (Meflin-Cre ${ }^{+/-}$; Rosa26-LSL-rtTA3 ${ }^{+/-}$; TRE-Meflin) mice fed with or without doxycycline supplementation were quantified by qPCR. The relative expression of Meflin (Islr) to Collal (Collal) was compared in the indicated mice.

(c) CAFs isolated from tumours developed in the indicated mice were cultured on Petri dishes for $24 \mathrm{~h}$ in the presence or absence of doxycycline. The cells were fixed and stained for Meflin mRNA using ISH. The graph on the right shows the quantification of Meflin mRNA in CAFs isolated from the indicated mice by qPCR.

(d) Tissue sections from EO771 tumours developed in control and Meflin TO mice were stained for CD31 to visualise tumour vessels (left), followed by quantification of the stained areas (right). The boxed regions are magnified in adjacent panels. 
Table 1. Characteristics of patients who received ICB monotherapies.

\begin{tabular}{|c|c|c|c|}
\hline \multirow[b]{2}{*}{ Variable } & \multicolumn{2}{|c|}{ Meflin expression } & \multirow[b]{2}{*}{ p-value } \\
\hline & High & Low & \\
\hline Median age - yr (range) & $69(43-80)$ & $70(42-85)$ & $0.920^{\dagger}$ \\
\hline Sex - no. (\%) & & & 0.663 \\
\hline Female & $23(36.5 \%)$ & $11(31.4 \%)$ & \\
\hline Male & $40(63.5 \%)$ & $24(68.6 \%)$ & \\
\hline Subtype - no. (\%) & & & 0.0813 \\
\hline Squamous cell carcinoma (LUSC) & $17(27.0 \%)$ & $3(8.6 \%)$ & \\
\hline Adenocarcinoma (LUAD) & $37(58.7 \%)$ & $27(77.1 \%)^{\|}$ & \\
\hline Others & $9(14.3 \%)$ & $5(14.3 \%)$ & \\
\hline Targetable EGFR mutation in LUAD - no. (\%) & & & $>0.999$ \\
\hline Not detected & $26(70.3 \%)$ & $17(68.0 \%)^{\|}$ & \\
\hline Detected & $11(29.7 \%)$ & $8(32.0 \%)$ & \\
\hline PD-L1 TPS ${ }^{\ddagger}$ - no. (\%) & & & 0.149 \\
\hline$<1 \%$ & $16(26.2 \%)$ & $7(20.6 \%)$ & \\
\hline $1-49 \%$ & $18(29.5 \%)$ & $17(50.0 \%)$ & \\
\hline $50 \% \leq$ & $27(44.3 \%)$ & $10(29.4 \%)$ & \\
\hline Brinkman index ${ }^{\S}$ - no. (\%) & & & 0.525 \\
\hline$<400$ & $26(41.3 \%)$ & $12(34.3 \%)$ & \\
\hline $400 \leq$ & $37(58.7 \%)$ & $23(65.7 \%)$ & \\
\hline ECOG-PS - no. (\%) & & & 0.255 \\
\hline $2 \leq$ (Poor) & $8(12.7 \%)$ & $8(22.9 \%)$ & \\
\hline 0 or 1 (Good) & $55(87.3 \%)$ & $27(77.1 \%)$ & \\
\hline Tx line - no. (\%) & & & 0.0046 \\
\hline $1 \mathrm{~L}$ or $2 \mathrm{~L}$ & $46(73.0 \%)$ & $15(42.9 \%)$ & \\
\hline $3 \mathrm{~L} \leq$ & $17(27.0 \%)$ & $20(57.1 \%)$ & \\
\hline
\end{tabular}

*: Fisher's exact test

$\dagger$ : Mann-Whitney U test

$\$$ : Tumour Proportion Score was the percentage of viable tumour cells showing partial or complete membrane staining at any intensity.

$\S$ : Brinkman index was defined as the number of cigarettes smoked per day multiplied by the number of years of smoking (e.g. if one smoked 20 cigarettes per day for 20 years, the Brinkman index is 400).

I: Only conventional cytotoxic chemotherapeutics were considered in the treatment line.

$10 \|$ : One patient with anaplastic lymphoma kinase (ALK)-rearranged NSCLC 
Table 2. Hazard ratios and p-values for multivariate Cox proportional hazard regression model analysis in NSCLC patients subjected to ICB monotherapies.

\begin{tabular}{|c|c|c|c|c|}
\hline Variable & $\begin{array}{l}\text { Hazard ratio }(95 \% \\
\text { CI )for OS }\end{array}$ & $\begin{array}{l}\mathrm{p} \text {-value for } \\
\text { OS }\end{array}$ & $\begin{array}{l}\text { Hazard ratio }(95 \% \quad \mathrm{CI}) \\
\text { for PFS }\end{array}$ & $\begin{array}{l}\text { p-value for } \\
\text { PFS }\end{array}$ \\
\hline Age & $0.960(0.933-0.989)$ & 0.0069 & $0.959(0.933-0.987)$ & 0.0038 \\
\hline Subtype & & $0.701^{*}$ & & $0.914^{*}$ \\
\hline LUSC & Reference & & Reference & \\
\hline LUAD & $0.796(0.300-1.62)$ & 0.401 & $0.827(0.320-2.14)$ & 0.696 \\
\hline Others & $0.746(0.264-2.11)$ & 0.580 & $0.907(0.319-2.58)$ & 0.856 \\
\hline PD-L1 TPS & & $0.105^{*}$ & & $0.856^{*}$ \\
\hline$<1 \%$ & Reference & & Reference & \\
\hline $1-49 \%$ & $1.02(0.460-2.24)$ & 0.971 & $1.08(0.576-2.04)$ & 0.804 \\
\hline $50 \% \leq$ & $1.92(0.906-4.08)$ & 0.0885 & $0.909(0.474-1.74)$ & 0.773 \\
\hline \multicolumn{5}{|c|}{ Targetable EGFR mutation } \\
\hline Not detected & $\mathrm{NI}^{\dagger}$ & & Reference & \\
\hline Detected & & & $2.25(0.987-5.12)$ & 0.0539 \\
\hline \multicolumn{5}{|l|}{ Brinkman index } \\
\hline$<400$ & Reference & & Reference & \\
\hline $400 \leq$ & $0.497(0.262-0.942)$ & 0.0320 & $0.684(0.351-1.33)$ & 0.264 \\
\hline \multicolumn{5}{|l|}{ ECOG-PS } \\
\hline $2 \leq$ (Poor) & Reference & & Reference & \\
\hline 0 or 1 (Good) & $0.0511(0.0224-0.117)$ & $<0.0001$ & $0.108(0.0479-0.242)$ & $<0.0001$ \\
\hline \multicolumn{5}{|l|}{ Tx line } \\
\hline $1 \mathrm{~L}$ or $2 \mathrm{~L}$ & Reference & & Reference & \\
\hline $3 \mathrm{~L} \leq$ & $1.09(0.586-2.02)$ & 0.791 & $0.854(0.485-1.50)$ & 0.583 \\
\hline \multicolumn{5}{|l|}{ Meflin } \\
\hline Low & Reference & & Reference & \\
\hline High & $0.420(0.220-0.800)$ & 0.0084 & $0.488(0.273-0.871)$ & 0.0152 \\
\hline
\end{tabular}

4 *: Wald test

$5 \uparrow$ : Targetable EGFR mutation status was not included in the multivariate analysis for OS because of the p-value in 6 the univariate analysis $(\mathrm{p}=0.290)$. 
1 Extended Data Table 1. Characteristics of NSCLC patients who underwent operations at 2 Nagoya University Hospital.

3

\begin{tabular}{|c|c|c|c|}
\hline \multirow[b]{2}{*}{ Variable } & \multicolumn{2}{|c|}{ Meflin expression } & \multirow[b]{2}{*}{ p-value* } \\
\hline & High & Low & \\
\hline Median age - yr (range) & $70(47-84)$ & $68.5(26-85)$ & $0.391^{\dagger}$ \\
\hline Sex - no. $(\%)$ & & & 0.659 \\
\hline Female & $14(33.3 \%)$ & $18(39.1 \%)$ & \\
\hline Male & $28(66.7 \%)$ & $28(60.9 \%)$ & \\
\hline Subtype - no. (\%) & & & 0.0002 \\
\hline Squamous cell carcinoma (LUSC) & $19(45.2 \%)$ & $4(8.7 \%)$ & \\
\hline Adenocarcinoma (LUAD) & $20(47.6 \%)$ & $39(84.8 \%)$ & \\
\hline Others & $3(7.1 \%)$ & $3(6.5 \%)$ & \\
\hline Targetable EGFR mutation in LUAD - no. (\%) & & & 0.0467 \\
\hline Not detected & $16(75.0 \%)$ & $19(50.0 \%)$ & \\
\hline Detected & $4(25.0 \%)$ & $19(50.0 \%)$ & \\
\hline Stage - no. $(\%)$ & & & 0.217 \\
\hline $\mathrm{I}$ & $24(57.1 \%)$ & $34(73.9 \%)$ & \\
\hline II & $11(26.2 \%)$ & $9(19.6 \%)$ & \\
\hline III & $7(16.7 \%)$ & $3(6.5 \%)$ & \\
\hline
\end{tabular}

4 *: Fisher's exact test

5 †: Mann-Whitney U test 
1 Extended Data Table 2. Hazard ratios and p-values for multivariate Cox proportional 2 hazard regression model analysis in NSCLC patients who underwent operations at Nagoya 3 University Hospital.

4

\begin{tabular}{|c|c|c|c|c|}
\hline Variable & $\begin{array}{l}\text { Hazard ratio }(95 \% \mathrm{CI}) \\
\text { for OS }\end{array}$ & $\begin{array}{l}p \text {-value } \\
\text { for OS }\end{array}$ & $\begin{array}{l}\text { Hazard ratio }(95 \% \mathrm{CI}) \\
\text { for DFS }\end{array}$ & $\begin{array}{l}\mathrm{p} \text {-value } \\
\text { for DFS }\end{array}$ \\
\hline Age & $1.036(0.995-1.08)$ & 0.0857 & $1.03(0.992-1.07)$ & 0.123 \\
\hline Subtype & & $0.856^{*}$ & & $0.889^{*}$ \\
\hline LUSC & Reference & & Reference & \\
\hline LUAD & $0.854(0.374-1.95)$ & 0.707 & $0.843(0.389-1.83)$ & 0.664 \\
\hline Others & $1.19(0.323-4.39)$ & 0.794 & $1.04(0.288-3.77)$ & 0.951 \\
\hline \multicolumn{5}{|c|}{ Targetable EGFR mutation } \\
\hline Not detected & Reference & & Reference & \\
\hline Detected & $0.836(0.298-2.35)$ & 0.733 & $1.33(0.555-3.18)$ & 0.523 \\
\hline Stage & $1.62(1.02-2.57)$ & 0.0404 & $1.66(1.07-2.58)$ & 0.0245 \\
\hline \multicolumn{5}{|l|}{ Meflin expression } \\
\hline Low & Reference & & Reference & \\
\hline High & $2.15(0.988-4.70)$ & 0.0537 & $1.60(0.806-3.18)$ & 0.179 \\
\hline
\end{tabular}


1 Extended Data Table 3. Characteristics of NSCLC patients from the TCGA-LUAD and 2 LUSC dataset.

3

\begin{tabular}{|c|c|c|c|}
\hline \multirow[b]{2}{*}{ Variable } & \multicolumn{2}{|c|}{ Meflin expression } & \multirow[b]{2}{*}{ p-value* } \\
\hline & High & Low & \\
\hline Median age - yr (range) & $68(38-87)$ & $66(39-88)$ & $0.0105^{\dagger}$ \\
\hline Sex - no. $(\%)$ & & & 0.115 \\
\hline Female & $160(37.0 \%)$ & $138(42.7 \%)$ & \\
\hline Male & $273(63.0 \%)$ & $185(57.3 \%)$ & \\
\hline Subtype -no. (\%) & & & $<0.0001$ \\
\hline LUSC & $309(71.0 \%)$ & $78(24.1 \%)$ & \\
\hline LUAD & $126(29.0 \%)$ & $245(75.9 \%)$ & \\
\hline Stage - no. (\%) & & & 0.580 \\
\hline I & $231(53.5 \%)$ & $183(56.7 \%)$ & \\
\hline II & $127(29.4 \%)$ & $84(26.0 \%)$ & \\
\hline III & $74(17.1 \%)$ & $56(17.3 \%)$ & \\
\hline
\end{tabular}

4 *: Fisher's exact test

$5 \uparrow$ : Mann-Whitney U test

6 
1 Extended Data Table 4. Hazard ratios and p-values for multivariate Cox proportional 2 hazard regression model analysis in TCGA-LUAD and -LUSC.

3

\begin{tabular}{|c|c|c|c|c|}
\hline Variable & $\begin{array}{l}\text { Hazard ratio }(95 \% \mathrm{CI}) \\
\text { for OS }\end{array}$ & $\begin{array}{l}\mathrm{p} \text {-value } \\
\text { for OS }\end{array}$ & $\begin{array}{l}\text { Hazard ratio }(95 \% \mathrm{CI}) \\
\text { for PFS }\end{array}$ & $\begin{array}{l}\mathrm{p} \text {-value } \\
\text { for DFS }\end{array}$ \\
\hline Age & $1.01(1.00-1.03)$ & 0.0385 & $1.01(1.00-1.02)$ & 0.0372 \\
\hline \multicolumn{5}{|l|}{ Subtype } \\
\hline LUSC & Reference & & Reference & \\
\hline LUAD & $0.941(0.722-1.23)$ & 0.653 & $1.16(0.917-1.46)$ & 0.218 \\
\hline Stage & $1.43(1.24-1.66)$ & $<0.0001$ & $1.34(1.17-1.52)$ & $<0.0001$ \\
\hline \multicolumn{5}{|c|}{ Meflin expression } \\
\hline Low & Reference & & Reference & \\
\hline High & $1.03(0.784-1.34)$ & 0.853 & $0.998(0.789-1.26)$ & 0.987 \\
\hline
\end{tabular}




\section{Methods}

2 Subject details This study was conducted in accordance with the Declaration of Helsinki and approved by the Ethics Committee of Nagoya University Graduate School of Medicine (approval number 2017-0127-3). We retrospectively enrolled two cohorts of non-small cell lung cancer (NSCLC) patients at Nagoya University Hospital to identify the effects of Meflin ${ }^{+}$cancer associated fibroblasts (CAFs) on patient outcomes. One cohort included patients with resectable NSCLC who underwent surgery (surgery cohort, $\mathrm{n}=147$; Extended Table 1). The other was a cohort of patients with advanced or recurrent NSCLC who received programmed cell death 1 (PD-1) or programmed cell death 1 ligand 1 (PD-L1) antibody-based immune checkpoint blockade (ICB) monotherapy (ICB cohort, $\mathrm{n}=132$, Table 1). All patients consented to the Institutional Review Board-approved protocols permitting specimen collection.

Clinical efficacy analysis Overall survival (OS) was defined as the time from surgery or the start of ICB therapy until death from any cause. Disease-free survival (DFS) was defined as the time from surgery until recurrence of cancer or death from any cause. Progression-free survival (PFS) was defined as the time from ICB monotherapy initiation until disease progression or death from any cause. Patient follow-up ended when an outcome was recorded or censored as of the database lock on 1st November 2018 for the surgery cohort or 30th April 2020 for the ICB cohort. Response to ICB was determined according to immunotherapy Response Evaluation Criteria in Solid Tumours (iRECIST) at each time point, which included iCR (complete response), iSD (stable disease), and iPR (partial response), as well as unconfirmed PD (iUPD) and confirmed PD (iCPD) (61). ORR was defined as the ratio of patients who achieved iPR or iCR. 
1 Tumour samples 88 of 147 patients from the surgery cohort and 98 of 132 patients from the ICB

2 cohort were included for further investigation due to their characteristics described in Extended

3 Data Figures 3A and 4A. All tumour tissues from the surgery cohort and 38 of 98 selected

4 patients from the ICB cohort were obtained at the time of surgery, and 60 tumour tissues from

5 the ICB cohort were obtained at the time of diagnostic biopsy or re-biopsy before ICB

6 monotherapy.

7

TCGA analysis We downloaded gene expression and clinical outcome data of TCGA lung adenocarcinoma (LUAD) and lung squamous cell carcinoma (LUSC) from cBioPortal for Cancer Genomics (https://www.cbioportal.org/) (62, 63). RNA-seq by Expectation-Maximization (RSEM) values were used to classify the patients into Meflin-high and -low groups with a cut-off value so that the ratios of Meflin-high and -low patients were similar to those of the surgery cohort in our institution.

Mice All mice were kept in specific pathogen-free conditions in the Division of Experimental Animals, Nagoya University Graduate School of Medicine. All experimental protocols were approved by the Animal Care and Use Committee of Nagoya University Graduate School of Medicine. The generation of an autochthonous lung adenocarcinoma mouse model (KP mice), Meflin-KO mice, and Meflin-Cre mice have been described previously $(30,37,44)$.

We generated a transgenic mouse line carrying a third-generation tetracycline-response element (TRE)-Meflin to induce Meflin expression in CAFs. To this end, the open reading frame (ORF) of the mouse Meflin gene was inserted into the multiple cloning site of a tetracyclineinducible expression vector pTRE3G (Clontech, 631168), followed by microinjection into fertilised eggs of C57BL/6 mice. We established four lines of TRE-Meflin with germline 
transmission. We selected one line for further experiments based on the confirmed doxycyclinemediated induction of Meflin expression in mouse embryonic fibroblasts (data not shown). The TRE-Meflin mice were crossed with Meflin-Cre and Rosa26-CAGs-LSL (LoxP-Stop-LoxP)rtTA3 knock-in mice (hereafter termed R26-rtTA3, JAX stock\# 029617, ref. 48).

Genomic DNA extracted from mouse tails was used for PCR-based genotyping. The primer sequences were as follows: Meflin-KO forward, 5'-GCTGCATTTGAGCTGAGCCTCTGG-3'; Meflin-KO reverse, 5'-AACCCCTTCCTCCTACATAGTTGG-3'; Meflin-Cre forward, 5'TAGGTGGTATTGGATTCTGGCTGGG-3'; Meflin-Cre reverse, 5'TTGAAGTAGTCGACGATGTCCTGG-3'; R26-rtTA3 forward, 5'TACTCAATGGAGTCGGTATCGAAGGC-3; R26-rtTA3 reverse, 5'CCAATACGCAGCCCAGTGTAAAGTGG-3'; TRE-Meflin forward, 5'GATCGCCTGGAGCAATTCCACAAC-3'; TRE-Meflin reverse, 5'CTGTTGGCtGACAGGCTCAGTGTGG-3'. The PCR product sizes from Meflin-KO, MeflinCre, R26-rtTA3, and TRE-Meflin alleles were 267, 385, 393, and 315 bp, respectively.

Cell lines MC-38 (Kerafast, ENH204), a murine colon cancer cell line, and EO771 (CH3 BioSystems, 94A001), a murine breast cancer cell line, were maintained in Dulbecco's modified Eagle's medium (DMEM, Gibco) and RPMI 1640 (Gibco), respectively, supplemented with 10\% heat-inactivated foetal bovine serum (FBS). All cell lines used were routinely screened for Mycoplasma contamination by 4, 6-diamidino-2-phenylindole (DAPI) staining.

Syngeneic tumour studies In vivo tumour studies were performed as follows: 6-week-old WT control and Meflin-KO female mice were inoculated subcutaneously in their right flanks with 1.0 $\times 10^{6} \mathrm{MC}-38$ cells suspended in $100 \mu \mathrm{L}$ of phosphate-buffered saline (PBS) or orthotopically in 
1 their fourth right mammary fat pads with $5.0 \times 10^{5}$ EO771 cells suspended in $50 \mu \mathrm{L}$ of PBS. The

2 volumes of MC-38 tumours were measured and calculated two to three times per week using the

3 modified ellipsoid formula as follows: $1 / 2 \times\left(\right.$ length $\times$ width $\left.^{2}\right)$. Mice with tumour volumes

$4>2,000 \mathrm{~mm}^{3}$ were sacrificed. Animals whose tumours were ulcerated with bleeding before

5 progression were terminated and included in the study.

7 In vivo antibody treatment To investigate the efficacy of ICB therapy in the MC-38

8 subcutaneous tumour model, anti-mPD-1 (BioLegend, RMP1-14) and isotype control

9 (BioLegend, RTK2758) antibodies were administered intraperitoneally to mice on day 4, which

10 was four days after tumour inoculation at a dose of $200 \mu \mathrm{g} / \mathrm{body}$, followed by subsequent

11 antibody administration on day 7 and 10 at the same dose. For EO771 orthotopic tumour models,

12 antibody administration was initiated on day 6 after palpable tumours had formed, followed by

13 antibody administration on day 9 and 12 at the same dose via the same route.

15 Tumour growth/tumour volume analysis A linear mixed-effects model was used to examine

16 repeated measurement data to investigate the effect of genotype (G: WT or Meflin-KO),

17 treatment (T: control or PD-1), and interaction between $\mathrm{G}$ and $\mathrm{T}$ on tumour volume over time.

18 The following model was used in the analysis:

19

$\log (\mathrm{TV})=\beta_{0}+\mathrm{b}_{0}+\left(\beta_{1}+\mathrm{b}_{1}\right) \times$ days $+\beta_{2} \times \mathrm{G}+\beta_{3} \times \mathrm{T}+\beta_{12} \times$ days $\times \mathrm{G}+\beta_{13} \times$ days $\times \mathrm{T}+\beta_{123} \times$

21 days $\times \mathrm{G} \times \mathrm{T}$

$22\left(\mathrm{~b}_{0}, \mathrm{~b}_{1}\right) \sim \operatorname{Normal}(0, \Phi), \Phi=\left(\sigma^{2}{ }_{\mathrm{B} 0}, \sigma_{\mathrm{B} 01}, \sigma^{2}{ }_{\mathrm{B} 1}\right)$ 
Here, $\beta_{0}, \beta_{1}, \beta_{2}, \beta_{3}, \beta_{12}, \beta_{13}$, and $\beta_{123}$ are the coefficients of the fixed effects. $b_{0}$ is the random

effect of the intercept, and $b_{1}$ is the random effect of the slope. $\sigma^{2}$ в0 is the variance of the individual difference at the baseline, $\sigma^{2}{ }_{\mathrm{B} 1}$ is the variance of the individual difference of the slope, and $\sigma_{\mathrm{B} 01}$ is the covariance of the individual difference of the baseline and the individual difference of the slope. Regression lines were used to fit a linear profile to the time courses of logarithm-transformed tumour volumes in each group. Fitting was performed using customised functions in R v.3.6.3, which integrates software from open-source packages, including lme4 and lmerTest $(64,65)$. Visualisation of the growth curves was performed using GraphPad Prism 6.

For EO771 orthotopic tumour models, we measured and analysed tumour volumes on the day of termination. Because the data showed non-normal distribution and heteroscedasticity, we chose a non-parametric Brunner-Munzel test with permutation to analyse tumour volumes in each group. The effect size was calculated using Cliff's delta statistic method and visualised using functions in R v.3.6.3, which integrates software from an open-source package, including dabestr (66).

Tumour processing. To isolate cells from tumours for FCM analysis, tumours were mechanically dissociated, followed by filtering through 100 and $40 \mu \mathrm{m}$ cell strainers and centrifugation to collect the cells. For quantitative PCR, total RNA was extracted from tumours using TRI-Reagent (Molecular Research Center), following the manufacturer's protocol. To isolate CAFs from tumours, tumours were mechanically minced and digested using the tumour dissociation kit (Miltenyi Biotec, 130-096-730) in gentleMACS C-Tubes (Miltenyi Biotec, 130093-237) according to the manufacturer's instructions. Tumour samples were minced and incubated in digestion media at $37^{\circ} \mathrm{C}$ for $30 \mathrm{~min}$ in a gentleMACS Octo dissociator (Miltenyi Biotec, 130-096-427). Following the digestion period, cells were suspended in a cold FACS 
1 buffer $(0.5 \%$ BSA and $2 \mathrm{mM}$ EDTA in PBS), filtered through $70 \mu \mathrm{m}$ filters, and centrifuged to 2 collect the cells.

4 Flow cytometry analysis FCM staining and analysis were performed using conventional procedures. Cells were washed using a FACS buffer (0.5\% BSA and 2 mM EDTA in PBS) and stained with cell-surface antibodies and Fixable Viability Dye eFlour 506 (eBioscience). For the intracellular staining of Foxp3 and Ki-67, cells were fixed and permeabilized using the Foxp3/Transcription Factor Staining Buffer Set (eBioscience) according to the manufacturer's instructions, followed by staining with monoclonal antibodies against Foxp3 (1:50 dilution) and Ki-67 (1:100 dilution). After washing, cells were analysed with a BD LSRFortessa X-20 flow

11 cytometer (BD Biosciences) and FlowJo (TreeStar) software. In this study, the following anti12 mouse antibodies labelled with fluorescent dyes were used: CD3-Alexa Fluor (AF) 700 (clone 17A2), CD4-APC-Fire 750 (clone RM4-4), CD8a-Brilliant Violet (BV) 785 (clone 53-6.7), CD25-BV605 (clone PC61), PD-1-BV421 (clone 29F.1A12), PD-L1-APC (clone 10F.9G2),

CD137-PE (clone 17B5), Tim-3-PE-Cyanine7 (clone RMT3-23), TIGIT-BV421 (clone 1G9), CD96-PE (clone 3.3), CD226-BV711 (clone TX42.1) (all from BioLegend); FoxP3-PerCPCyanine5.5 (clone FJK-16s), Ki-67-FITC (clone SolA15), ICOS-APC (clone 7E.17G9) (all from eBioscience); LAG-3-BV711 (BD Bioscience, clone C9B7W); 2B4-AF488 (Novus Biologicals, clone 244F4).

Quantitative PCR (qPCR) Total RNA was purified from whole tumours and cultured CAFs using the RNeasy Mini Kit (Qiagen) according to the manufacturer's instructions. Purified RNA samples were reverse-transcribed using ReverTra Ace (Toyobo) with oligo dT and random primers. qPCR of the generated cDNAs was performed with TaqMan Gene Expression Master Mix (Applied Biosystems) using a StepOnePlus thermal cycler (Applied Biosystems). Applied 
Biosystems synthesises customised TaqMan probes and primers for the mouse Meflin (Islr) coding sequence. Cycling conditions were as follows: $50^{\circ} \mathrm{C}$ for $2 \mathrm{~min}, 95^{\circ} \mathrm{C}$ for $10 \mathrm{~min}, 40$ cycles of $95^{\circ} \mathrm{C}$ for $15 \mathrm{~s}$, and $60^{\circ} \mathrm{C}$ for $1 \mathrm{~min}$. The data were analysed using the $2^{-\Delta \Delta \mathrm{Ct}}$ method and normalised to Colla1 (Mm00801666_g1).

RNA in situ hybridisation To detect single mRNA molecules, an RNA in situ hybridisation (ISH) assay based on RNAscope technology (ACD, Advanced Cell Diagnostics) was performed on formalin-fixed and paraffin-embedded (FFPE) human and mouse tissue samples and fixed cultured cells. Sample preparation was performed according to the manufacturer's protocol for RNAscope on tissue samples (ACD, document number 322452) or the technical note for fixed cultured cells (ACD, document number MK-50010). ISH was performed according to the protocol of the RNAscope 2.5 HD Detection Reagent-BROWN (ACD, 322310), the 2.5 HD Duplex Detection Kit (ACD, 322500), or Multiplex Fluorescent Reagent Kit v2 (ACD, 323100). Briefly, FFPE sections were deparaffinized in xylene and 100\% ethanol and dried completely for 5 min at room temperature (RT). After incubation with $\mathrm{H}_{2} \mathrm{O}_{2}$ solution for $10 \mathrm{~min}$ at RT, slides were treated in a boiling target retrieval solution $\left(>98^{\circ} \mathrm{C}\right)$ for $15 \mathrm{~min}$, washed in distilled water, dehydrated in 100\% ethanol, and dried completely. Finally, the slides were incubated with Protease Plus (ACD) at $40^{\circ} \mathrm{C}$ for $30 \mathrm{~min}$. After washing in distilled water, slides were incubated with the relevant probes at $40^{\circ} \mathrm{C}$ for $2 \mathrm{~h}$, followed by amplification through sequential amplification using AMP-1 to AMP-6 reagents (ACD). Staining was visualised with 3,3diaminobenzidine (DAB) followed by counterstaining with haematoxylin. To detect the expression of more than two mRNAs on the same slides using bright field microscopy, we used the 2.5 HD Duplex Detection Kit (Fast Green and Fast Red, ACD), staining with alkaline phosphatase (ALP), and DAB followed by counterstaining with haematoxylin. To detect the expression of more than two mRNAs on the same slides by fluorescent microscopy, we used Multiplex Fluorescent Reagent Kit v2 (ACD), staining with fluorophores TSA (tyramide signal 
amplification)- cyanine 3 and - cyanine 5 (AKOYA Bioscience), followed by counterstaining with DAPI. In this study, the following six different probes against genes of interest were used: Hs-ISLR (human Meflin) (NM_005545.3, region 275 - 1322; Cat No. 455481), Mm-Islr (mouse Meflin) (NM_012043.4, region 763 - 1690; Cat No. 450041), Hs-ACTA2 (NM_001613.2, region 10 - 1341; Cat No. 311811), Hs-PDGFRA (NM_006206.4, region 844-1774; Cat No. 604481), and Hs-PDPN (NM_001006625.1, region 911-2045; Cat No. 539751).

\section{IHC assay using the Opal-IHC kit and immunofluorescent staining combined with}

RNAscope For IHC, FFPE tissue sections were deparaffinized and subjected to antigen retrieval using a target retrieval solution (Dako or Novocastra) at $\mathrm{pH} \mathrm{6,7,} \mathrm{or} 9$ for $30 \mathrm{~min}$ in an electric kettle, followed by IHC using conventional procedures. For multiplex immunofluorescent (IF) staining of TILs, the Opal 7 Tumor Infiltrating Lymphocyte Kit (Akoya Bioscience, Cat No. OP7TL3001KT) was used to stain CD4, CD8, CD20, FoxP3, CD45RO, and pan-cytokeratin, according to the manufacturer's instructions. The sections were mounted using PermaFluor Aqueous Mounting Medium (Thermo Fisher Scientific, TA-030-FM), followed by scanning using a Vectra slide scanner (Akoya Bioscience). Five randomly selected multispectral highpowered field images of each section were captured using an automated imaging system (Vectra ver. 3.0, Akoya Bioscience), and loaded into user-trainable image analysis software (InForm, Akoya Bioscience), which allows the automated recognition of tissue regions and individual cells to perform cell classification and phenotyping.

For the combined detection of mRNAs by ISH and proteins by IF, we first stained FFPE sections by ISH with the fluorophore TSA-Cyanine 5 for visualisation. The ISH-stained slides were washed with PBS twice and incubated with blocking buffer containing $10 \%$ serum (of the host from which secondary antibodies were derived) for $30 \mathrm{~min}$ at RT, followed by incubation with primary antibodies diluted in PBS at $4{ }^{\circ} \mathrm{C}$ overnight. After washing with PBS, the slides 
were incubated with Alexa Fluor 488/594-conjugated secondary antibodies (Thermo Scientific) for 30 min at RT, followed by incubation with DAPI for $30 \mathrm{~s}$ at RT and mounting with PermaFluor aqueous mounting medium (Thermo Scientific).

For proteins for which corresponding antibodies that work for IF after performing ISH are unavailable, such as LCA and podoplanin (Extended Data Fig. 2A and B), we first performed IF using Opal fluorophores (Akoya Biosciences, NEL810001KT) according to the manufacturer's instructions. Briefly, FFPE sections were deparaffinized, followed by antigen retrieval in AR6 or AR9 (Akoya Biosciences, AR600250ML, AR900250ML) for 15 min at $98^{\circ} \mathrm{C}$. After blocking with Antibody Diluent/Blocking (Akoya Biosciences, ARD1001EA), the sections were incubated with primary antibodies for $2 \mathrm{~h}$ at RT or overnight at $4{ }^{\circ} \mathrm{C}$, washed three times in Tris-buffered saline (150 mM NaCl, $25 \mathrm{mM}$ Tris- $\mathrm{HCl})$ with $0.05 \%$ polyoxyethylene (20) sorbitan monolaurate (Tween 20) (TBST), and incubated in Opal Polymer HRP secondary antibody (Akoya Biosciences, ARH1001EA) for 10 min at RT. After three washes in TBST, Opal 570 in 1X Plus Amplification Diluent (1: 125) was added and reacted for $10 \mathrm{~min}$ at RT. After three washes in TBST, we performed ISH using RNAscope Multiplex Fluorescent Reagent Kit v2 (ACD, 323100) according to the manufacturer's instructions. For mRNA retrieval and the removal of antibody-HRP complexes, the slides were immersed in $1 \mathrm{X}$ Target Retrieval Buffer using a pressure cooker (SR-MP300, Panasonic) for $15 \mathrm{~min}$, followed by washing the sections in distilled water and dehydrating them in $100 \%$ ethanol. After drying the slides for $5 \mathrm{~min}$ at $60{ }^{\circ} \mathrm{C}$, they were treated with Proteinase Plus (ACD, 322331) for $30 \mathrm{~min}$ at $40{ }^{\circ} \mathrm{C}$. The sections were washed once in deionised water, incubated with target probes for $2 \mathrm{~h}$ at $40{ }^{\circ} \mathrm{C}$, washed twice in 1X wash buffer, and then incubated in amplification reagents (AMP1-3), followed by HRP-C1 Reagent (ACD, 323104). The signals were amplified with a TSA-Plus Cyanine 5 System (NEL745001KT, PerkinElmer), followed by mounting the sections with PermaFluor Aqueous Mounting Medium. Fluorescence was examined using an inverse immunofluorescence microscope BZ-X710 (Keyence) with optical sectioning. 
The following antibodies were used in the present study: mouse monoclonal anti-E-cadherin (BD Biosciences, clone 36, dilution 1:500), mouse monoclonal anti-human CD31 (Dako, clone JC70A, dilution 1:250), mouse monoclonal anti-LCA (Dako, clone 2B11, PD7/26), rabbit polyclonal anti-Col1a1 (Novus, NB600-408, dilution 1:500), mouse monoclonal anti- $\alpha-S M A$ (Dako, clone 1A4, dilution 1:500), mouse monoclonal anti-Podoplanin (Abcam, clone D2-40, dilution 1:100), and rat monoclonal anti-mouse CD31 (Dianova, clone SZ31, dilution 1:100).

Statistical analysis We used GraphPad Prism 6 or R v.3.6.3 for statistical analysis. Patient characteristics and binary outcomes were compared between the two groups using Fisher's exact test or the Mann-Whitney U test. Survival was analysed using the Kaplan-Meier approach and the log-rank Mantel-Cox test, as well as the Cox proportional hazards regression model. Variables with p-values $<0.2$, on the univariate Cox models, were included in the multivariate analyses. Also, Meflin expression status, PD-L1 TPS, and the variables with p-values $<0.1$ on the analyses of patient characteristics were included in the final model, irrespective of their statistical significances. The magnitudes of the associations were summarised using hazard ratios with $95 \%$ confidence intervals (CIs). To evaluate the predictive value for responders, a receiver operating characteristic curve (ROC) for discrete variables was created by plotting the truepositive rate against the false-positive rate at each threshold setting. The area under the curve (AUC) shown in the plot summarises the performance of discrete variables. The cut-off value of discrete variables in which the sum of sensitivity and specificity was the maximum was detected. For murine experiments, all data are representative of at least two to three independent experiments with three to six mice in each in vivo experiment. The data were expressed as means with 95\% CIs, unless otherwise specified. The relationships between groups were compared using a two-tailed unpaired Student's t-test with Welch's correction, unless otherwise specified. For multiple testing, The Holm-Bonferroni method was employed. Survival was analysed using 
1 the Kaplan-Meier approach and the log-rank Mantel-Cox test. Statistical significance was set at P $2<0.05$. 


\section{References}

2 61. Seymour, L. et al. iRECIST: Guidelines for response criteria for use in trials testing

3 immunotherapeutics. Lancet Oncol. 18, e143-e152 (2017).

4 62. Cerami, E. et al. The cBio Cancer Genomics Portal: An Open Platform for Exploring

5 Multidimensional Cancer Genomics Data. Cancer Discov. 2, 401-404 (2012).

6 63. Gao, J. et al. Integrative analysis of complex cancer genomics and clinical profiles using the $7 \quad$ cBioPortal. Sci. Signal. 6, pl1 (2013).

8 64. Bates, D., Mächler, M., Bolker, B. \& Walker, S. Fitting linear mixed-effects models using 9 lme4. J. Stat. Softw. 67, 1-48 (2015).

10 65. Kuznetsova, A., Brockhoff, P. B. \& Christensen, R. H. B. lmerTest package: Tests in linear 11 mixed effects models. J. Stat. Softw. 82, 1-26 (2017).

12 66. Ho, J., Tumkaya, T., Aryal, S., Choi, H. \& Claridge-Chang, A. Moving beyond P values: data 13 analysis with estimation graphics. Nat. Methods 16, 565-566 (2019). 
Figures

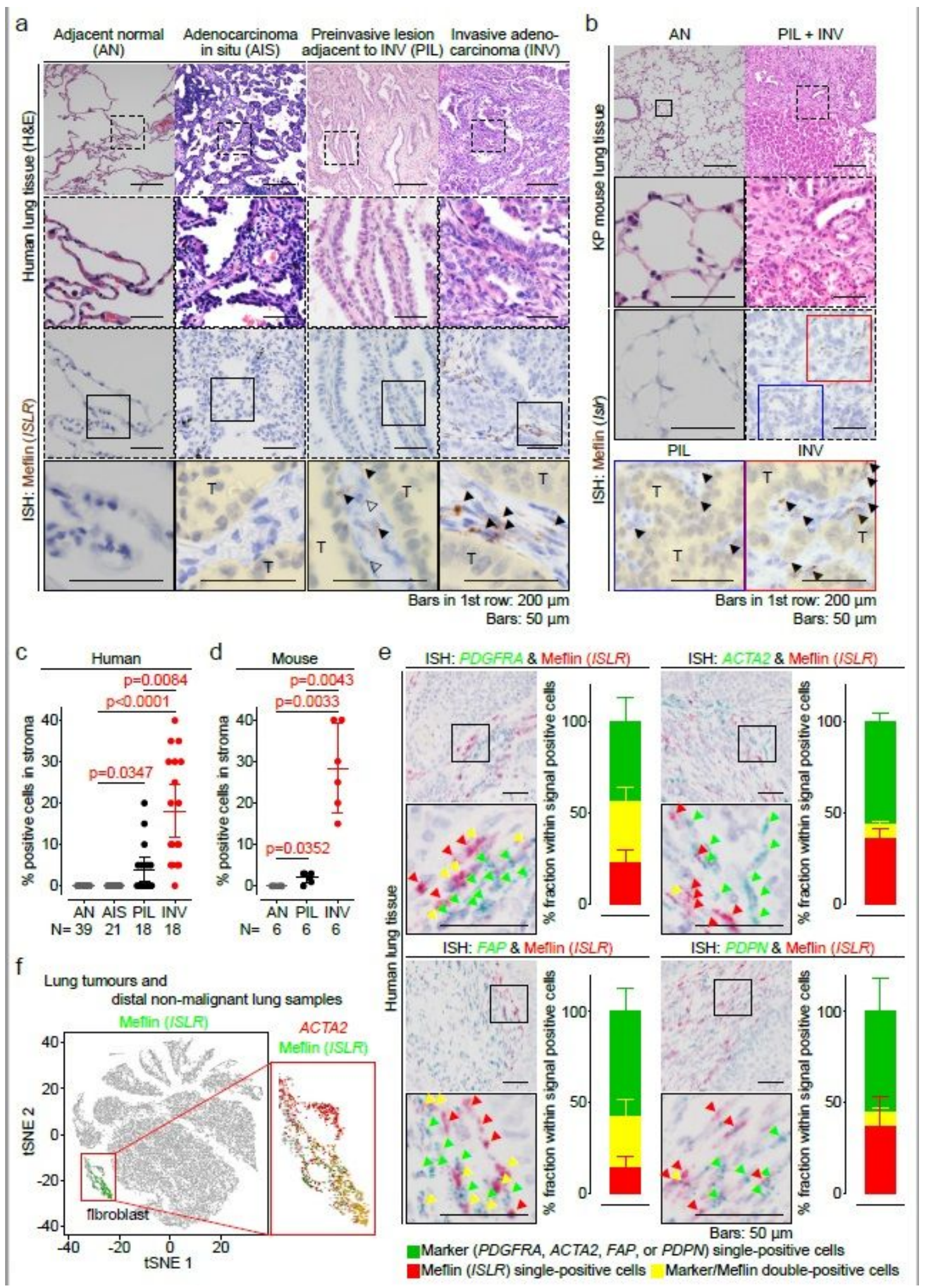

\section{Figure 1}

Meflin is a marker of CAFs that appear in invasive human NSCLC (a) Tissue sections derived from noninvasive (AIS) and invasive (INV) LUAD tumours were stained for H\&E (top panels) and Meflin mRNA by ISH (lower panels). Preinvasive lesions adjacent to the invasive tumour (PIL) and adjacent normal tissue 
(AN) were also examined. Boxed areas were magnified in lower panels. Black and open arrowheads denote Meflin+ stromal cells and Meflin- macrophages that phagocytise foreign material, respectively. Areas filled with yellow indicate tumour parenchyma comprised of tumour cells ( $T$ ). (b) Preinvasive (PIL) and invasive (INV) lesions of a tumour developed in the autochthonous LUAD mouse model (KP mice) were examined for Meflin expression by ISH. Meflin+ stromal cells (arrowheads) were found in the INV and PIL lesion, but not in adjacent normal tissue (AN). (c, d) Quantification of the percentage of Meflin+ cells in all cells with oval- to spindle-shaped nuclei found in the stroma of the normal (AN), non-invasive (AIS), preinvasive (PIL) and invasive (INV) cases and lesions of human LUAD cases (c) and the KP mouse model (d), respectively. All stromal cells found in high-power fields (400x) of the indicated number of cases were evaluated. (e) Duplex ISH for Meflin (red) and other CAF markers (green) showed that Meflin is variably co-expressed with other established CAF markers (PDGFRA, ACTA2, FAP, and PDPN) in CAFs of human NSCLC. Green, yellow, and red arrow heads denote cells that are single-positive for the indicated CAF markers, double-positive for the indicated CAF markers and Meflin, and single-positive for Meflin, respectively. All stromal cells that were positive for ISH signals in high-power fields from 3 independent NSCLC tumours were evaluated and quantified. (f) A single-cell analysis showing the specific expression of Meflin in fibroblasts in human NSCLC and distal non-malignant lung samples. The magnified image of the fibroblast fractionshowed that Meflin is expressed in some but not all fibroblasts, and that Meflin+ CAFs constitute a unique CAF subset distinct from a-SMA+CAFs. 

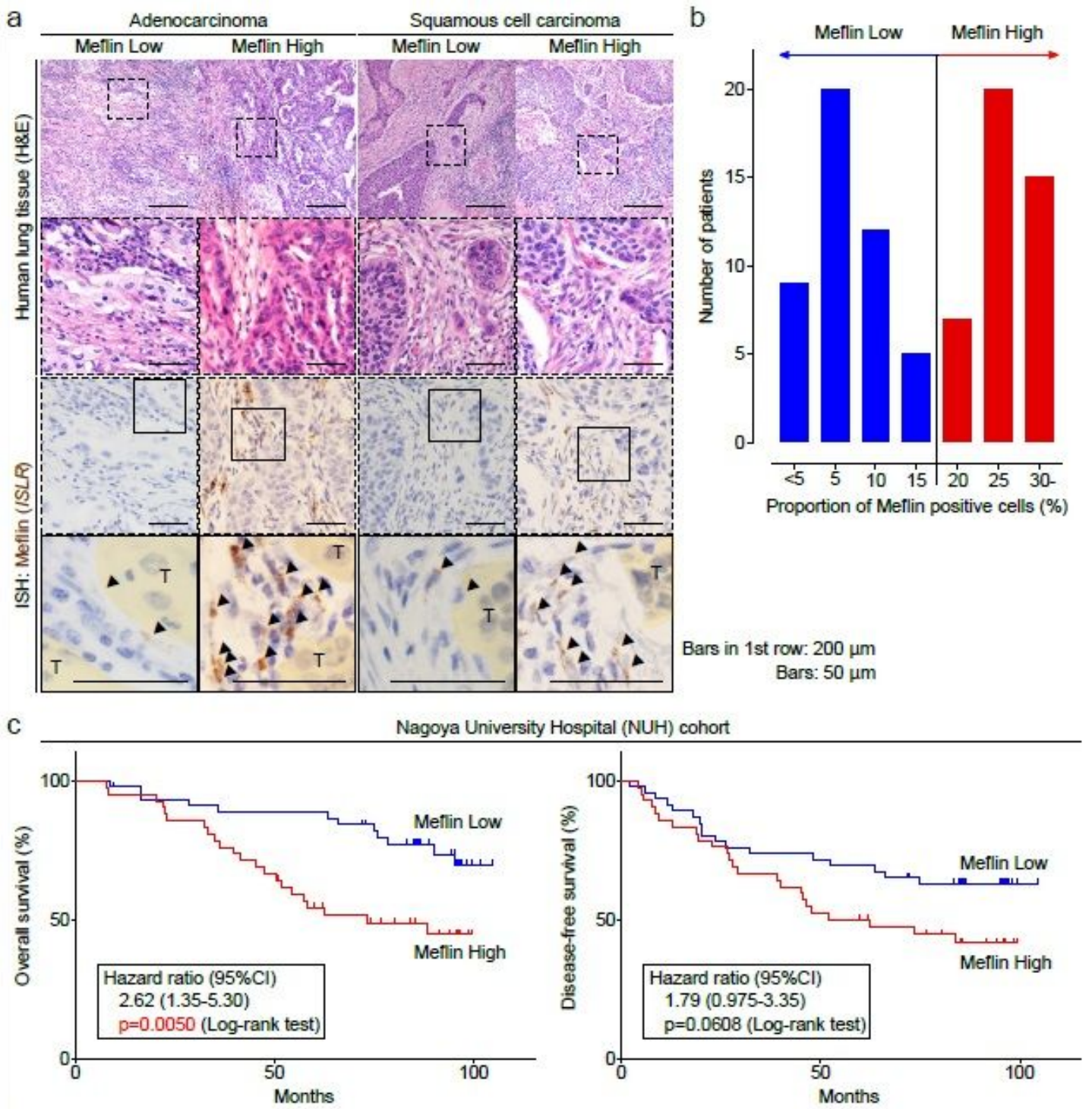

d

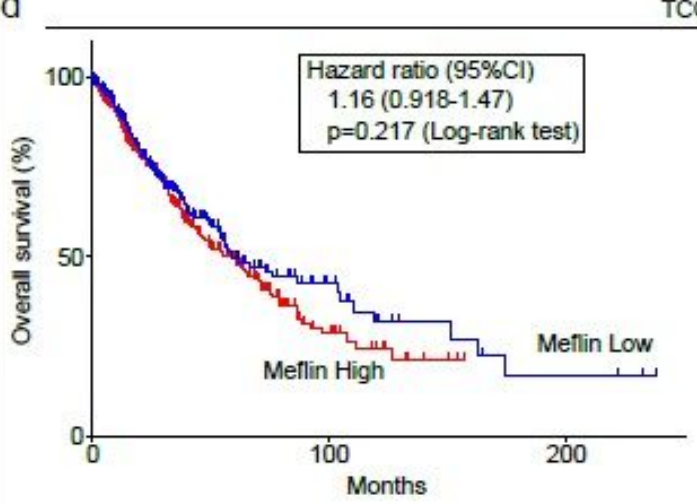

TCGA cohort

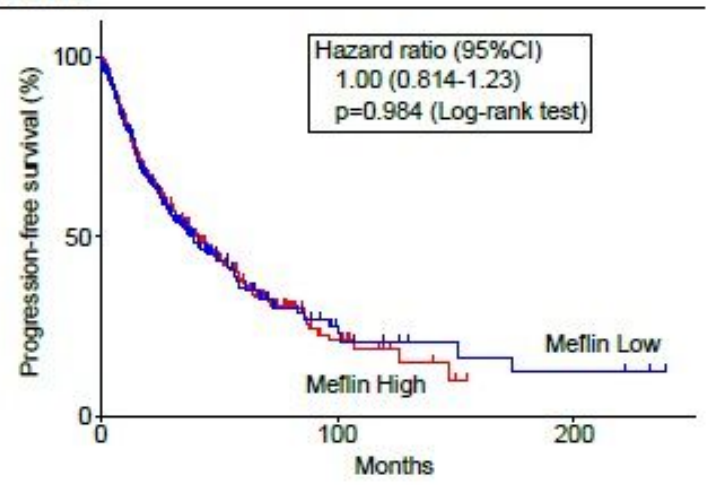

Figure 2

Infiltration of Meflin + CAFs is associated with poor outcomes of NSCLC patients (a) Representative images of Meflin-high and -low cases of invasive adenocarcinoma (left) and squamous cell carcinoma (right). Dashed boxed areas were magnified in middle panels, which showed serial sections stained for H\&E and Meflin mRNA by ISH. In the images of Meflin ISH, boxed areas were magnified in the lowest panels. Arrowheads indicate Meflin+ CAFs. (b) Distribution of NSCLC patients stratified by the percentage 
of Meflin+ CAFs in all stromal cells. The number of Meflin+ CAFs was counted in randomly selected five high-power microscopic fields. The proportion of Meflin+ CAFs was represented as the ratio of Meflin+ CAFs to all stromal cells with oval to spindle-shaped nuclei. Meflin-high was defined as $\geq 20 \%$ of stromal cells positive for Meflin. (c) Comparison of the outcomes of Meflin-high and -low NSCLC patients in our institution. Eighty-eight cases were classified according to Meflin expression, and OS (left) and DFS (right) of the cases were plotted by Kaplan-Meier analysis. (d) Effects of Meflin expression on NSCLC patients' outcomes analysed by using data sets available in the TCGA database. Seven hundred fiftyeight cases were classified into Meflin-high and -low groups, and OS (left) and PFS (right) were plotted by Kaplan-Meier analysis. Cut-off values were set at the top 33 and 80 percentiles for the expression of Meflin in LUAD and LUSC, respectively. 
a

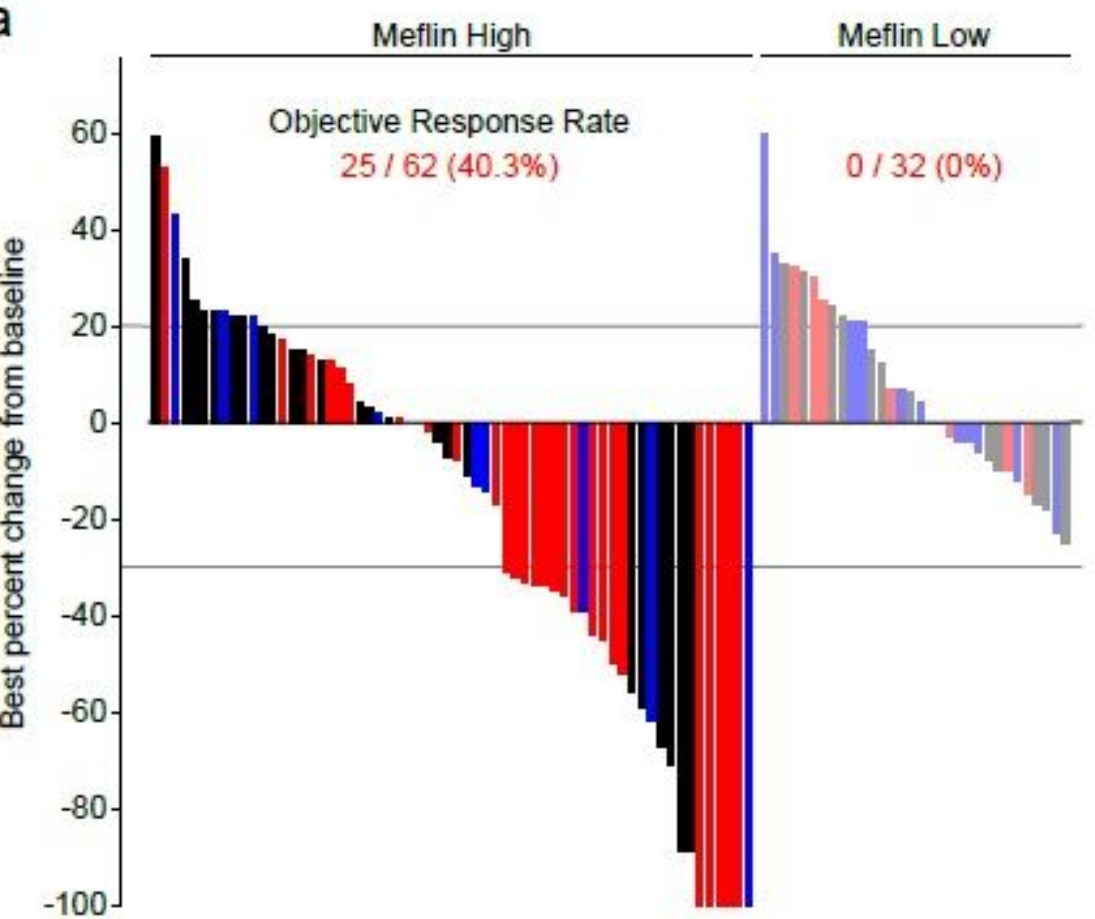

$p<0.0001$ (Fisher's exact test)

Nivolumab
Pembrolizumab

Atezolizumab

b

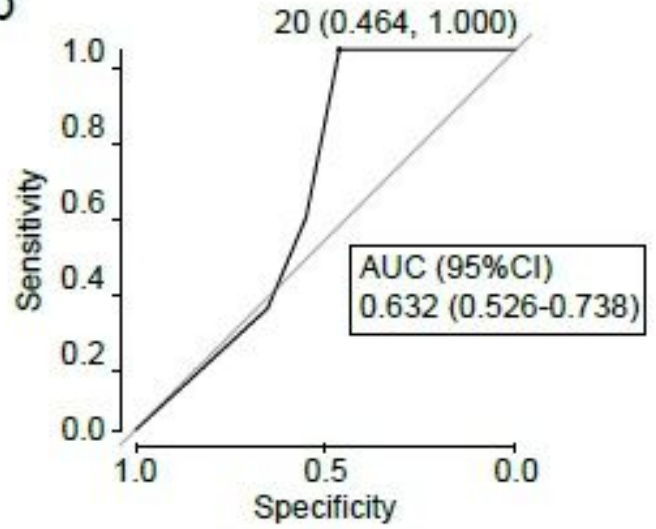

C

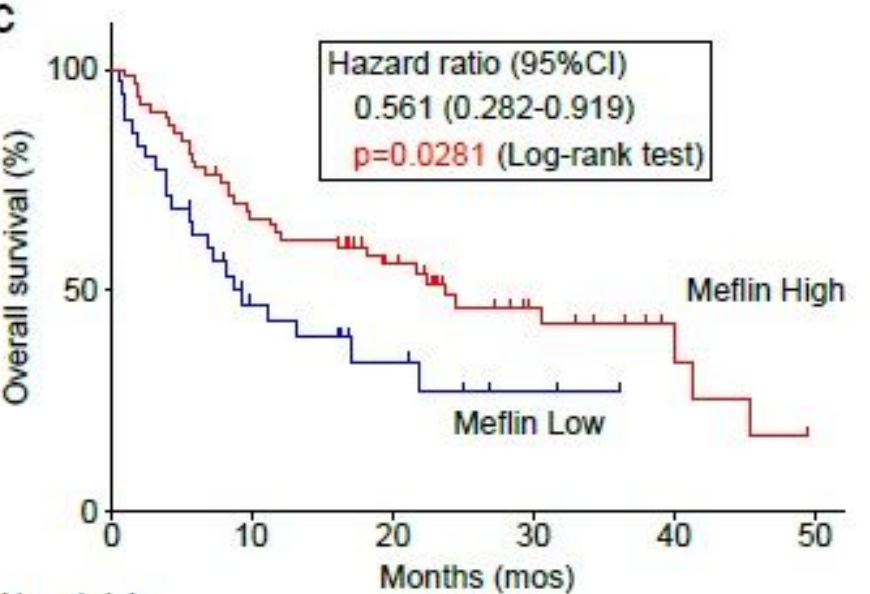

No. at risk

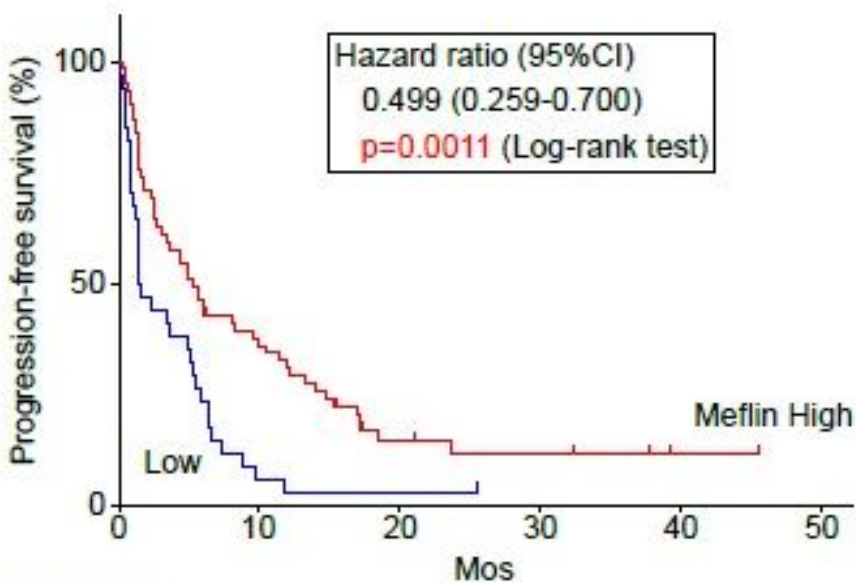

High 63

$\begin{array}{lll}41 & 27 & 13\end{array}$

50

Low 35

13

62

$0 \quad 0$

No. at risk

$\begin{array}{ccccccc}\text { High } & 62 & 21 & 6 & 4 & 1 & 0 \\ \text { Low } & 34 & 2 & 1 & 0 & 0 & 0\end{array}$

\begin{tabular}{|lcc|}
\hline Meflin & High & Low \\
No. of events (\%) & $35(55.6 \%)$ & $22(62.9 \%)$ \\
Mos, median (95\%Cl) & $23.6(12.1-41.3)$ & $9.34(5.52-21.9)$ \\
\hline
\end{tabular}

\begin{tabular}{|lcc|}
\hline Meflin & High & Low \\
No. of events $(\%)$ & $52(83.9 \%)$ & $33(97.1 \%)$ \\
Mos, median $(95 \% \mathrm{Cl})$ & $5.29(2.76-9.60)$ & $1.48(1.08-5.10)$ \\
\hline
\end{tabular}

Figure 3

NSCLC patients with high Meflin + CAF infiltration exhibit favourable response to ICB therapy (a) A waterfall plot showing changes in tumour size from baseline determined according to iRECIST criteria in Meflin-high (left) and -low (right) NSCLC patients who receive ICB monotherapy. Black, red and blue bars indicate patients treated with nivolumab, pembrolizumab, and atezolizumab, respectively. (b) A receiver operating characteristics (ROC) curve for the percentage of Meflin+ CAFs in tumour stroma. The ROC curve was obtained by plotting sensitivity against 1 - specificity at each threshold setting. The area under 
the curve (AUC) $(0.632 ; 95 \% \mathrm{Cl}, 0.526$ - 0.738) shown in the plot summarises the performance of Meflin+ CAFs in tumour stroma. (c) OS (left) and PFS (right) of Meflin-high (red) and -low (blue) NSCLC patients treated with ICB therapy. Meflin-high group showed a favourable response to ICB therapy compared to Meflin-low group. Shown in the boxes below the plots are the observed numbers of events (deaths or disease progression) and median survival (months) of Meflin-high and -low groups over the follow-up periods. Mos, months.

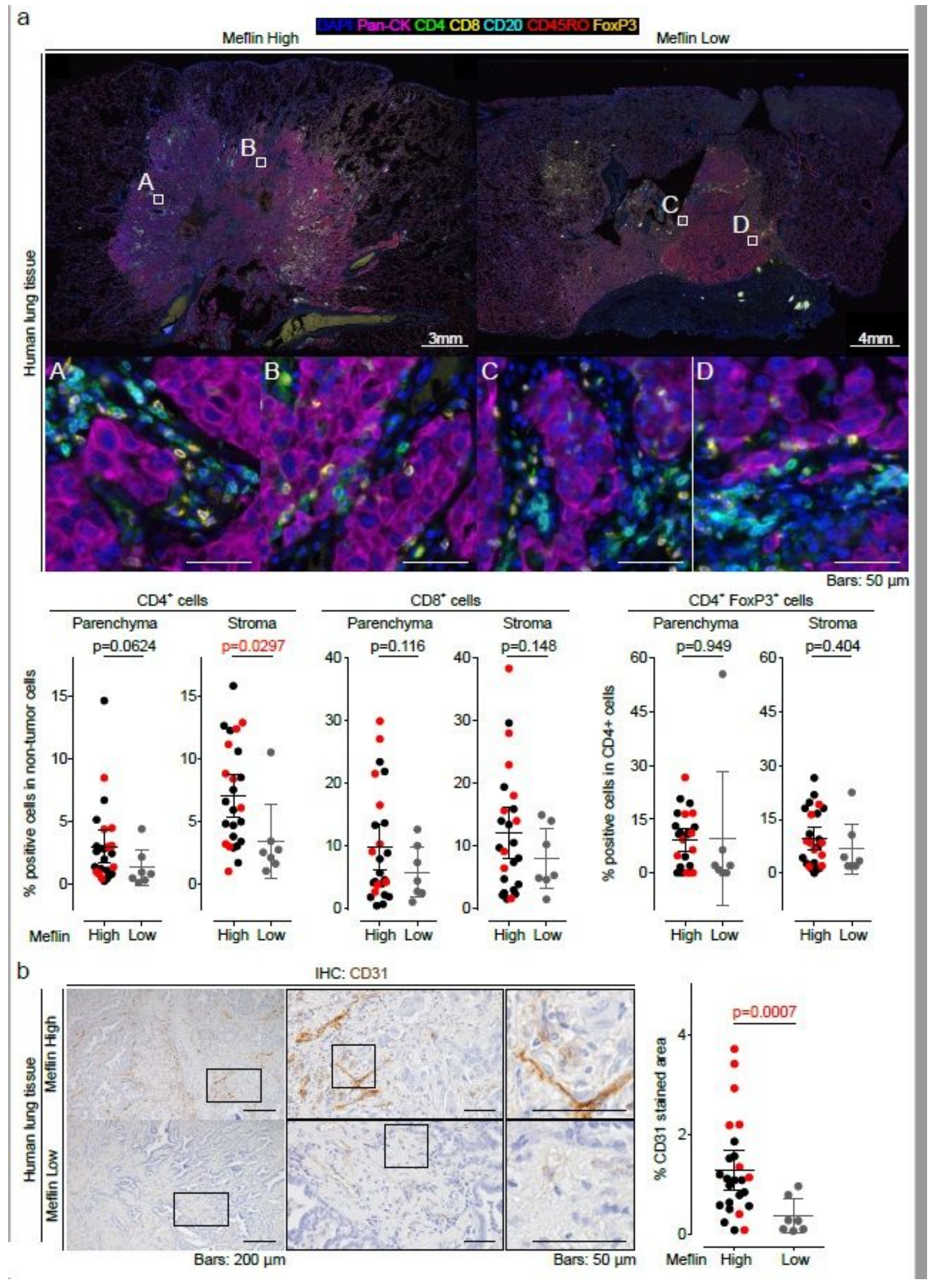

Figure 4 
Meflin expression in CAFs correlates with tumour infiltration of CD4+ T cells and vascular area in NSCLC patients treated with ICB monotherapy(a) Tissue sections prepared from tumours of NSCLC patients who received ICB monotherapy were stained by multiplex immunofluorescence for the indicated TIL markers, nuclei and pan ytokeratin (Pan-CK), followed by imaging with a multispectral imaging system.

Representative images of Meflin-high (left, $n=25$ ) and -low (right, $n=7$ ) cases are shown. Boxed regions were magnified in lower panels. The lower panels' graphs show the quantification of the percentage of CD4+, CD8+ and CD4+FoxP3+ T cells in all TILs infiltrated in intra-tumour (parenchyma) and stroma regions of each group. Red dots in the graphs denote the responders to ICB monotherapy. (b) Representative images of tissue sections of Meflin-high (upper panel) and -low (lower panel) tumours stained for CD31. The graph on the right shows the quantification of tumour vessel areas in each group following the method described in Extended Data Fig. 7. High magnification views randomly selected from 25 Meflin-high and 7 Meflin-low cases were analysed and quantified. Red dots in the graphs denote the responders to ICB monotherapy. 
a

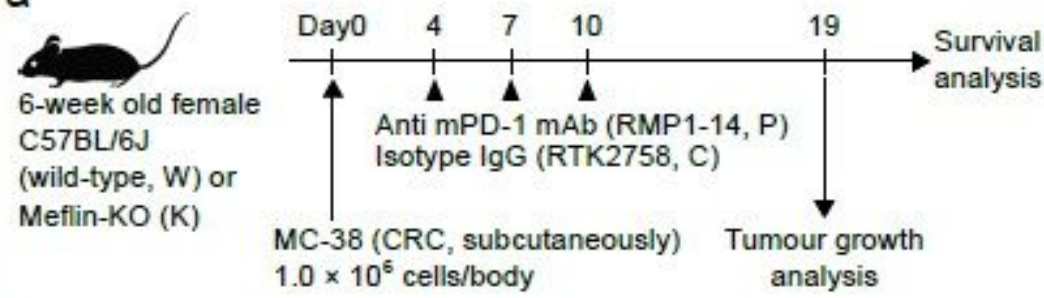

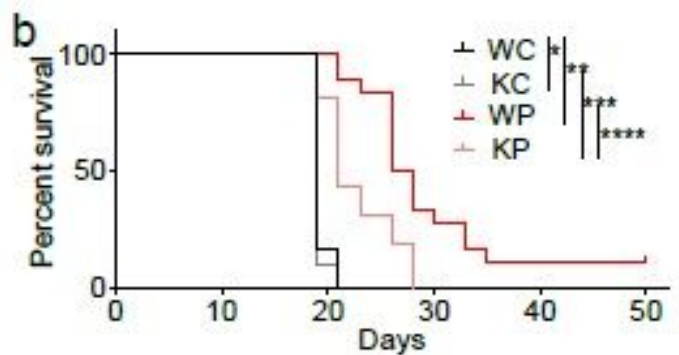

c

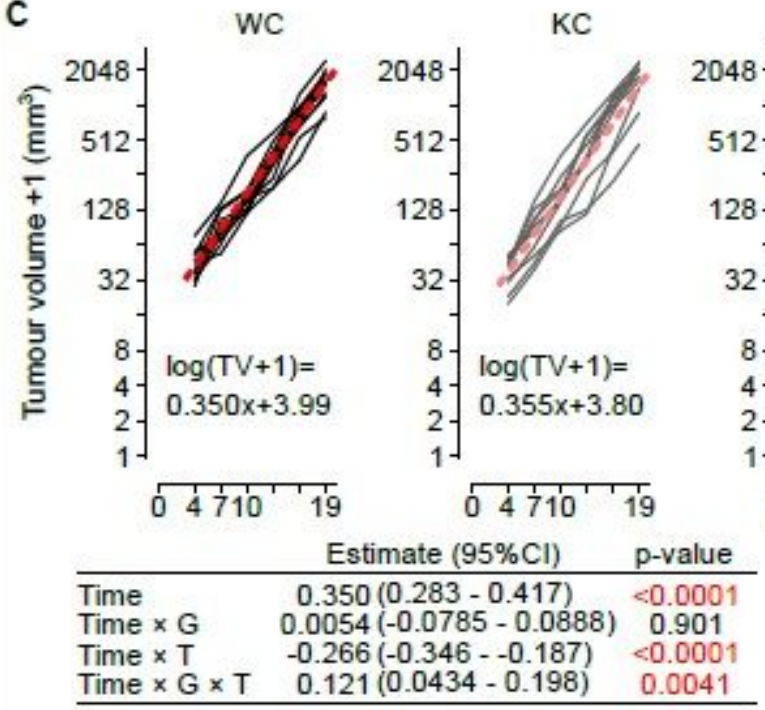

G: Genotype (wild-type (W) or Meflin-KO $(K)$ )

$\mathrm{T}$ : Treatment (control (C) or mPD-1 (P))

e
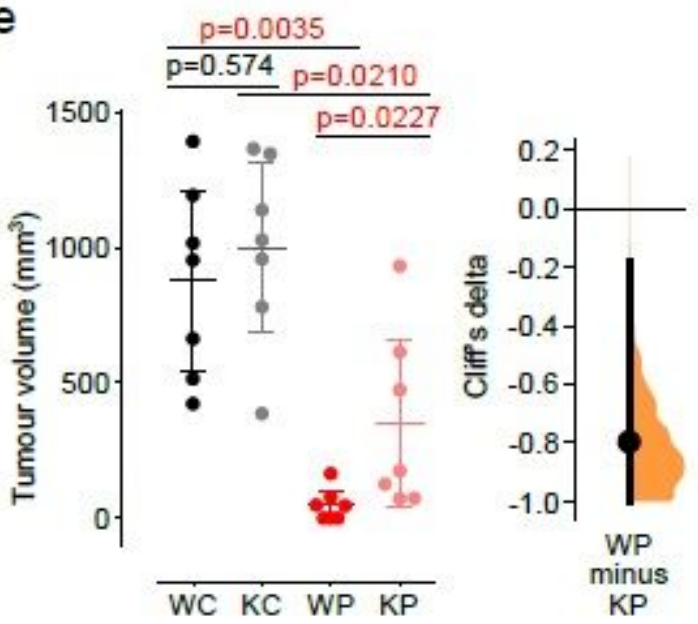

WP $\log (T V+1)=$

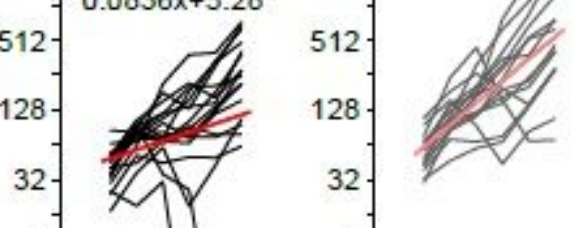

$\log (T V+1)=$

$0.210 x+5.10$ $0.0836 x+5.28$ 2048

$$
0 4 \frac { 1 7 0 } { 0 1 9 } \longdiv { 4 7 1 0 } 1 9 \text { Days }
$$

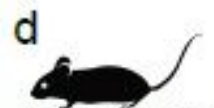

6-week-old female C57BL/6J (W) or Meflin-KO (K)

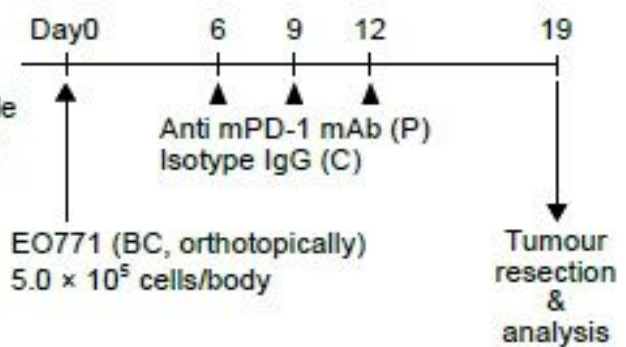

IHC: CD31
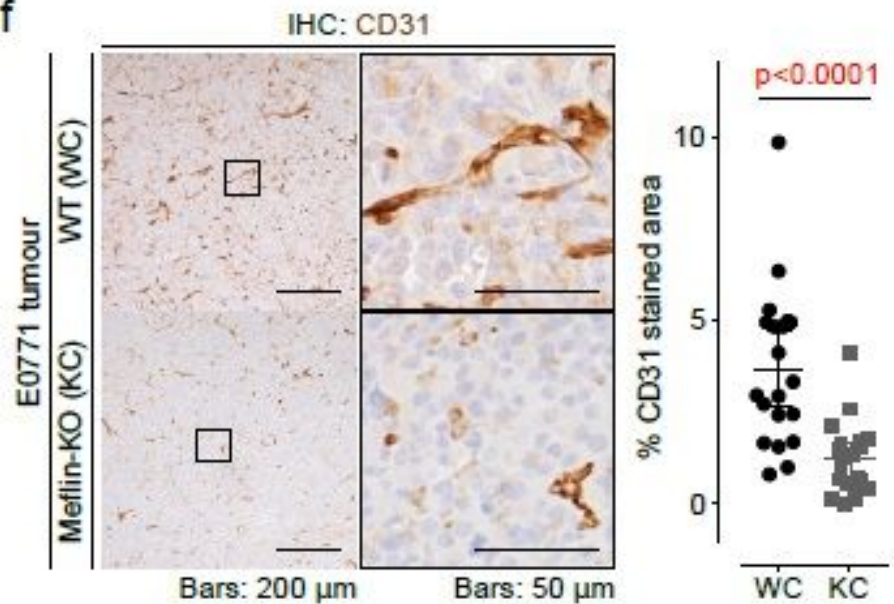

Figure 5

Tumours developed in Meflin-KO mice exhibit poor response to anti-mPD-1 therapy (a) An experimental protocol to test the anti-tumour effect of anti-mPD-1 therapy in mice. MC-38 mouse CRC cells were transplanted into C57BL/ 6 wild-type $(\mathrm{W})$ or Meflin-KO $(\mathrm{K})$ mice at day 0 , followed by the intraperitoneal administration of anti-mPD-1 (P) or an isotype control antibody (C). (b) Survival of wild-type (W) and Meflin-KO (K) mice non-treated (WC and KC, respectively) or treated with anti-PD-1 antibody (WP and KP, respectively). The numbers of mice tested for WC, KC, WP and KP groups were 12, 10, 18 and 16, 
respectively. ${ }^{\star}, p=0.658 ; * \star, p<0.0001 ; * \star \star, p=0.0033 ; * \star \star \star, p=0.0033$. (c) Time courses of the volumes of tumours developed in the indicated groups (black and grey lines). For the log transformation of tumour volumes, one was added to every tumour volume. Red lines indicate linear approximations. The table shown under the graphs shows the restricted maximum likelihood estimates of each parameter in a linear mixed-effects model that includes the interactions of time, time and mouse genetic background (G), time and anti-mPD-1 therapy $(T)$, and time and $G$ and $T$, while adding variable effects to the slope and intercept for each individual. (d, e) E0771 mouse BC cells were transplanted into wild-type (W) or Meflin-KO (K) mice at day 0 , followed by the intraperitoneal administration of anti-mPD-1 antibody (P) or an isotype control antibody (C). The number of mice tested for each group was 7. Shown in (e) are the quantification of tumour volume of each group (left) and the non-parametric estimate of effect size calculated by Cliff's delta (right). (f) Tissue sections from E0771 tumours developed in wild-type (WC) and Meflin-KO (KC) were stained for CD31 to visualise tumour vessels (left), followed by the quantification of the stained areas (right). Boxed regions were magnified in adjacent panels. 


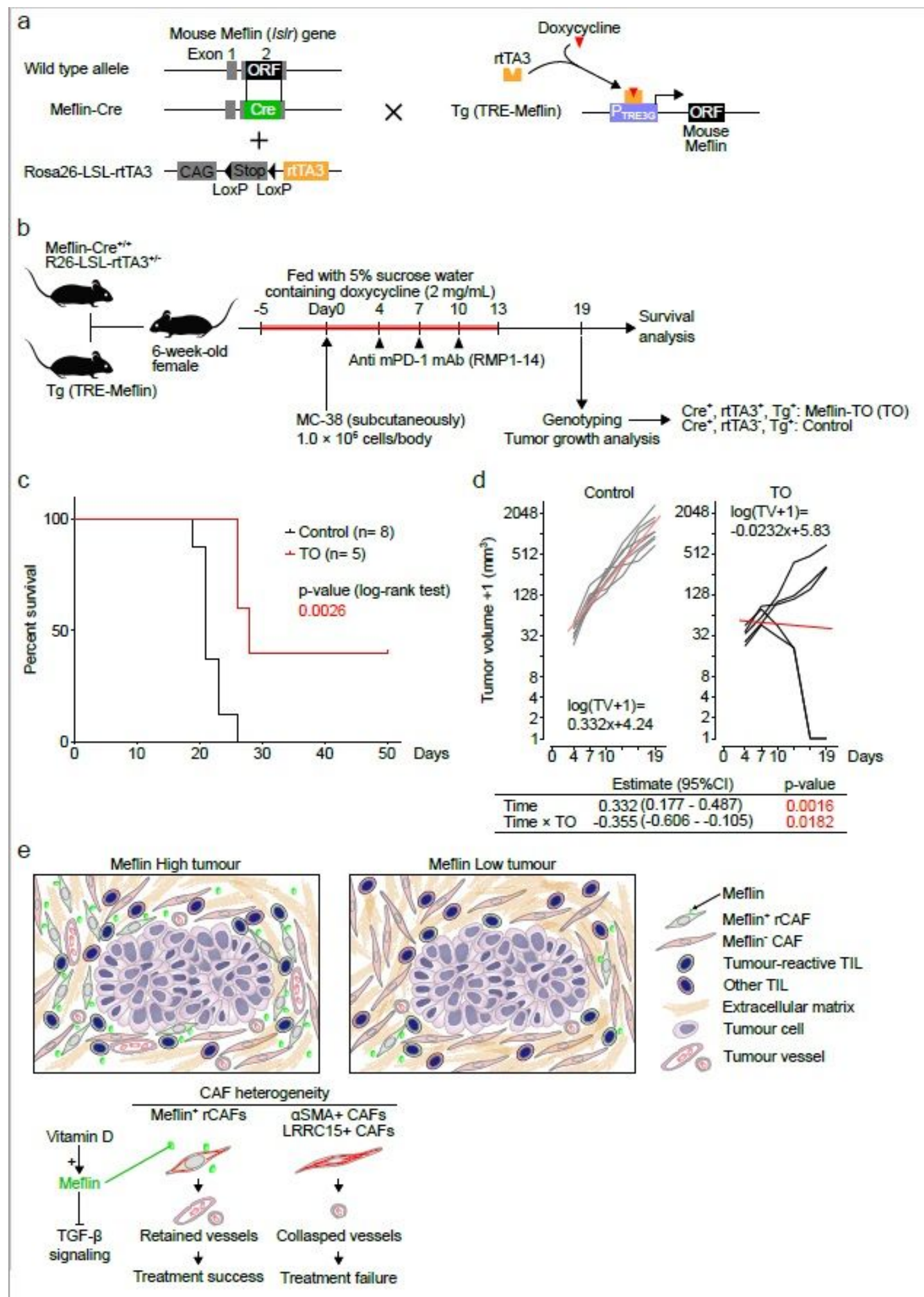

\section{Figure 6}

Induced expression of Meflin in CAFs improves tumour response to anti-mPD-1 therapy (a) A diagram of the generation of the mouse line carrying Meflin-Cre, which constitutively expresses Cre under the control of the Meflin promoter, Rosa26-LSL-rtTA3, and Tg (TRE Meflin) alleles that exhibit induced Meflin expression in Meflin-lineage cells upon doxycycline administration. ORF; open reading frame, CAG; chicken ß-actin promoter, Stop; stop element, rtTA3; the third-generation reverse tetracycline-regulated 
transactivator, Tg; transgenic, TRE; tetracycline-response element. (b) Meflin-Cre+/+; Rosa26-LSL-rtTA3+/mice were crossed with TRE-Meflin transgenic mice. The resultant 6-week-old female mice fed with doxycycline were subcutaneously implanted with MC-38 cells, followed by anti-mPD-1 therapy, genotyping, and tumour analysis. Mice that harbour all of the Meflin-Cre, Rosa26-LSL-rtTA3 and TREMeflin alleles were termed Meflin TO (Tet-on) mice.(c) Survival of control (Meflin-Cre+/-; Rosa26-LSLrtTA3-/-; TRE-Meflin) and Meflin TO (Meflin-Cre+/-; Rosa26-LSL-rtTA3+/-; TRE-Meflin) mice treated with anti-mPD-1 antibody. (d) Time courses of the volumes of tumours developed in the indicated groups (black and grey lines). For the log transformation of tumour volumes, one was added to every tumour volume. Red lines indicate linear approximations. The table shown under the graphs shows the restricted maximum likelihood estimates of each parameter in a linear mixed-effects model that includes the interactions of time and time and induced Meflin expression (TO), while adding variable effects to the slope and intercept for each individual. (e) Graphical summary and working hypothesis for CAF heterogeneity and its role in ICB response. Our data demonstrated that infiltration of Meflin+ CAFs was associated with perfused 